\title{
Study of Air Ingress Across the Duct During the Accident Conditions
}

\section{Reactor Concepts RD\&D}

Dr. Yassin Hassan

Texas A\&M University

Madeline Feltus, Federal POC Chang Oh, Technical POC

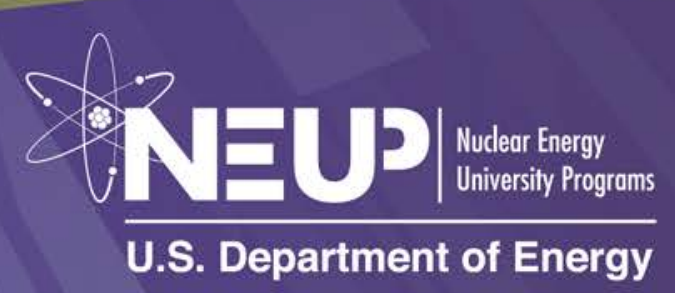




\section{Study of Air ingress across the duct during the accident conditions}

\section{Al[M TEXAS A\&M

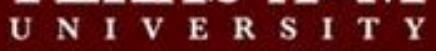

Covering Period: November 2009 - October 2012

Date of Report: October, 2013

Recipient: Name: Texas A\&M University

Address: 3133 TAMU

City: College Station

State: Texas

Zip: $\quad 77843$

Contract Number: 90521

Project Number: 09-841

Principal Investigator: Yassin Hassan - 9798457090 - y-hassan@tamu.edu Project Objective:

The objectives of this study are:

1) Particle Image Velocimetry (PIV) Measurement of Multiphase during Air Ingress:

We will measure the temperature distribution and concentration profile of the two species will be investigated using state-of-the-art particle image velocimetry (PIV) and laser induced fluorescence (LIF) techniques.

Experiments will be performed in a specially designed small test facility that will allow us to measure the velocity and temperature during stratifications and inflow/outflow behavior through the broken duct. Hot-wire anemometers will also be inserted into the duct and the tanks to obtain local velocity values. These experiments will provide new full-field data of temperature, concentration and velocity components with a high degree of spatial and temporal resolution. The data will allow us to better understand the fundamental flow features and develop improved models.

2) Computational Fluid Dynamics (CFD) Analysis of Air Ingress Phenomena:

We will construct advanced CFD models that incorporate the multiple physical phenomena associated with air ingress including as molecular 
diffusion, natural circulation and buoyancy. Modeling of convection is important owing to its potential to contribute to severe fuel and core oxidation. Turbulence modeling will also be employed under the various flow regimes of laminar mixed conditions. We will also utilize our developed large eddy simulation capabilities to capture the multiscales of flow structure. The measured data will provide a unique framework to validate the results of these CFD modeling efforts.

TPOCs: Chang Oh phone: 208-526-7716 email:Chang.Oh@inl.gov

Federal POC: Madeline Feltus phone: 301-903-2308 email:madeline.feltus@nuclear.energy.gov

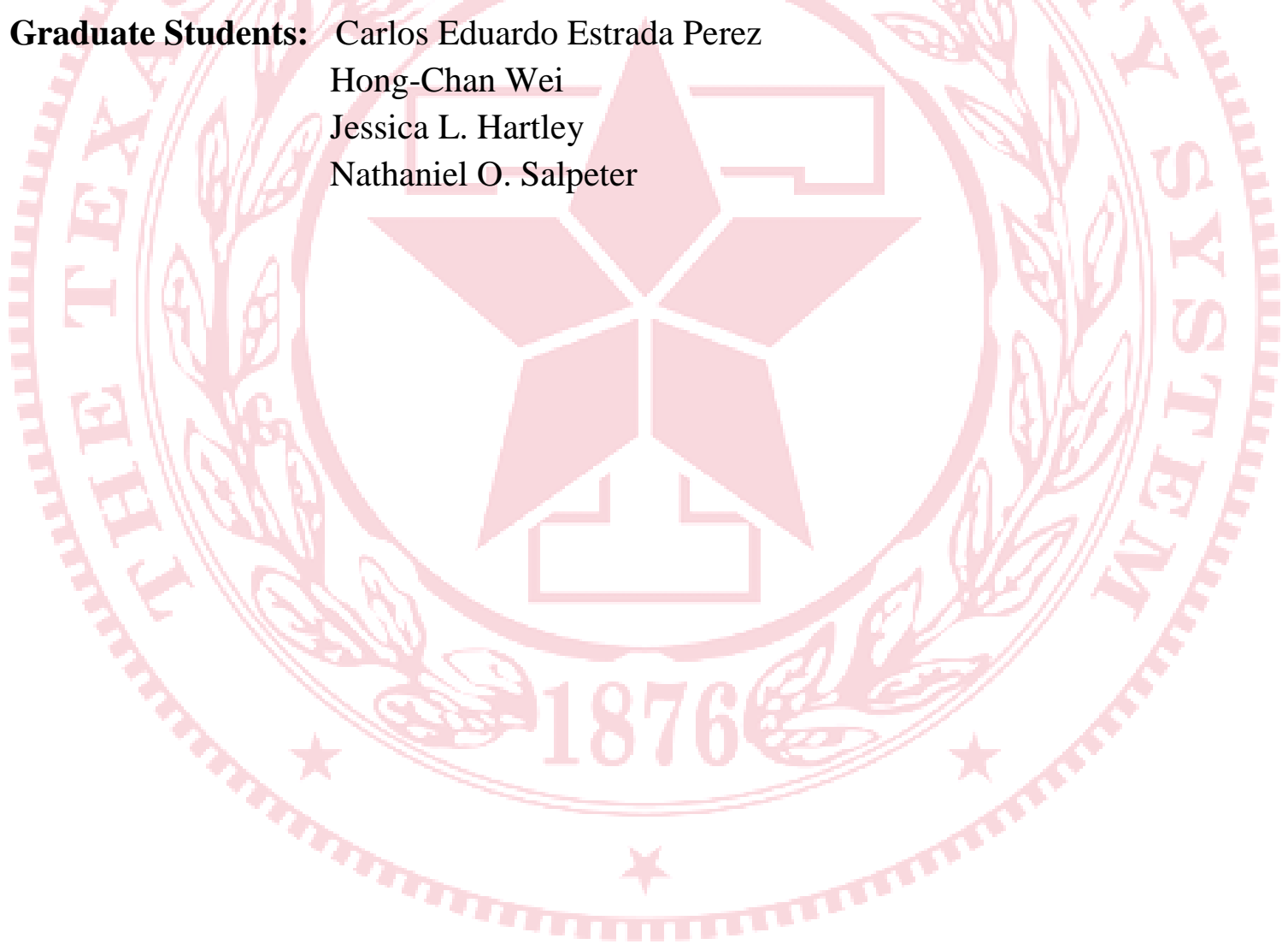




\section{ABSTRACT}

This research contains experimental and simulation works in order to study the air ingress phenomenon. Many cases with using liquid-liquid and gas-gas fluid-pairs were used to investigate the flow behaviors. The first task is the comprehensive work of experiments, and the second task focuses on the computational fluid dynamics (CFD) simulations. The double ended guillotine break leading to density-driven air ingress has been identified as a low probability yet high consequence event for Very High

Temperature Reactor (VHTR). The lower plenum of the VHTR contains the core support structure and is composed of graphite. During an air ingress event, oxidation of the graphite structure under high temperature conditions in an oxygen containing environment could degrade the integrity of the core support structure. Following this large break, air from the reactor containment will begin to enter the lower plenum via two mechanisms: diffusion or density driven stratified flow. The large difference in time scales between the mechanisms leads to the need to perform high fidelity experimental studies to investigate the dominant air ingress mechanism. A scaled test facility has been designed and built that allows the acquisition of velocity measurements during stratification after a pipe break. A non-intrusive optical measurement technique provides full-field velocity measurement profiles of the two species particle image velocimetry. The data allow a more developed understanding of the fundamental flow features, the development of improved models, and possible mitigation strategies in such a scenario.

Two brine-water experiments were conducted with different break locations. Flow fronts were analyzed and findings concluded that the flow has a constant speed through the pipe after the initial lock exchange. The time in which the flow enters the lower plenum is an important factor because it provides the window of opportunity for mitigation strategies in an actual reactor scenario. For both cases the flow of the heavier density liquid (simulating air ingress from the reactor containment) from the pipe enters the reactor vessel in under 6 seconds.

The diffusion velocity and heavy flow front of the stratified flow layer were compared for the $\mathrm{SF}_{6} / \mathrm{He}$ gas case. It is seen that diffusion plays less of a role as the transport mechanism in comparison to the density-driven stratified flow since the velocity of the diffusion is two orders of magnitude smaller than the velocity of the stratified flow mechanism. This is the reason for the need for density-driven stratified flow investigations following a loss of coolant accident. 
These investigations provided high-quality data for computational fluid dynamics validation in order for these models to depict the basic phenomena occurring in an air ingress scenario.

The objective of the simulations focuses on investigating the Kelvin-Helmholtz instability of the gravity-driven stratified flows inside a coaxial pipe. Richardson extrapolation was used for the grid independent study. The simulation results show good agreements with the experiments. Wavelet analysis and Proper Orthogonal Decomposition (POD) were used to study the flow behaviors and flow patterns.

In order to study the characteristic frequency of the air-ingress phenomenon, Brunt-Vaisala frequency, or buoyancy frequency, predicts a frequency of $2.34 \mathrm{~Hz}$; this is confirmed by the dominant frequency of $2.4 \mathrm{~Hz}$ obtained from the wavelet analysis between times $1.2 \mathrm{~s}$ and $1.85 \mathrm{~s}$. Generally, wavelet analysis shows much better performance than POD, in the air-ingress phenomenon, for a strongly transient scenario. Based on this study, when the fluid pair in a real condition is used, the time which air intrudes into the reactor is predictable.

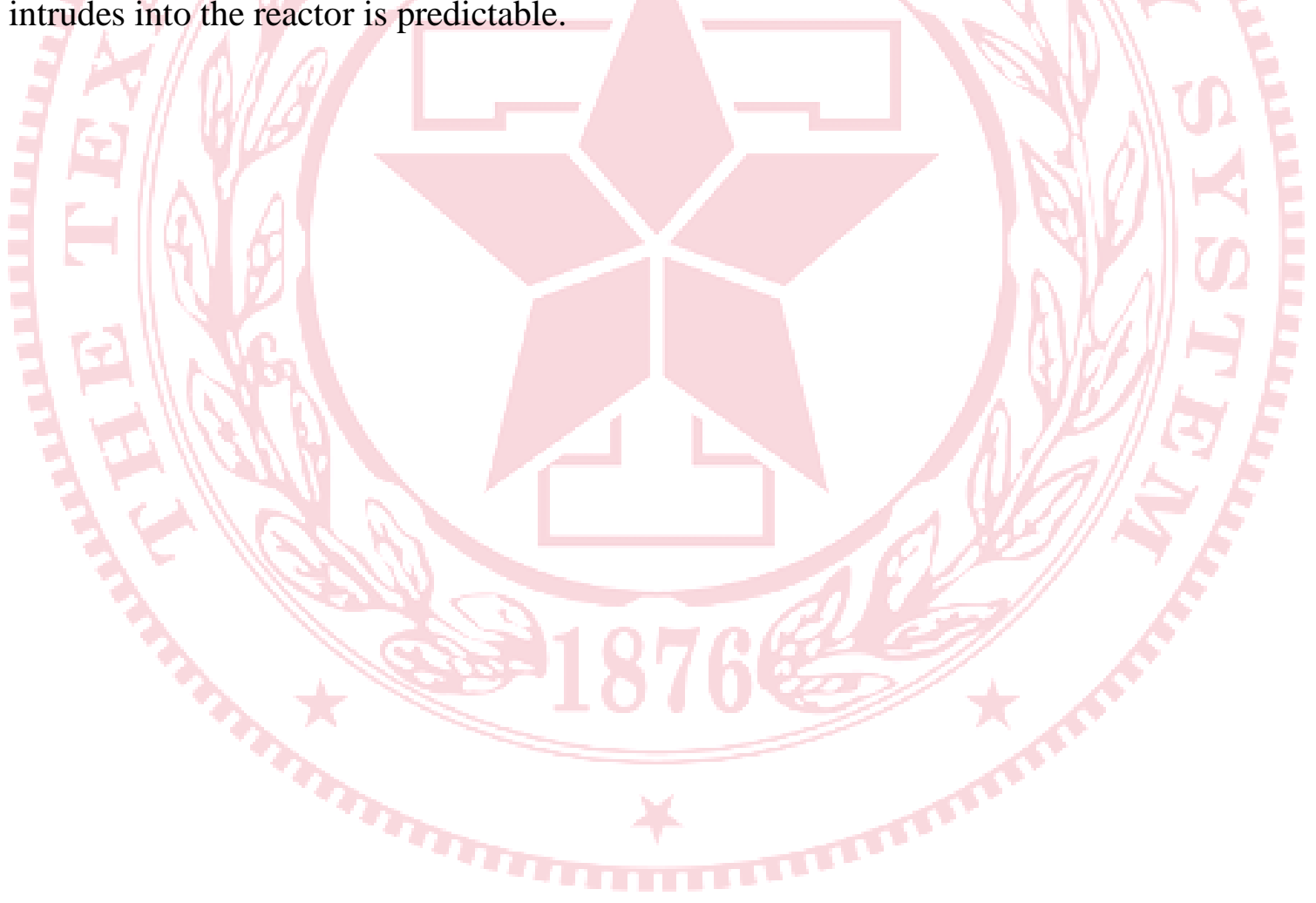




\section{ACKNOWLEDGEMENTS}

The project was founded by the Nuclear Energy University Program (NEUP) and the US Department of Energy (DOE).

\section{Students Involvement:}

1. Carlos Eduardo Estrada Perez - PhD Mechanical Engineering (in progress)

2. Hong-Chan Wei - PhD Nuclear Engineering (2012)

3. Jessica L. Hartley - MS Mechanical Engineering (2012)

4. Nathaniel O. Salpeter - PhD Mechanical Engineering (2012)

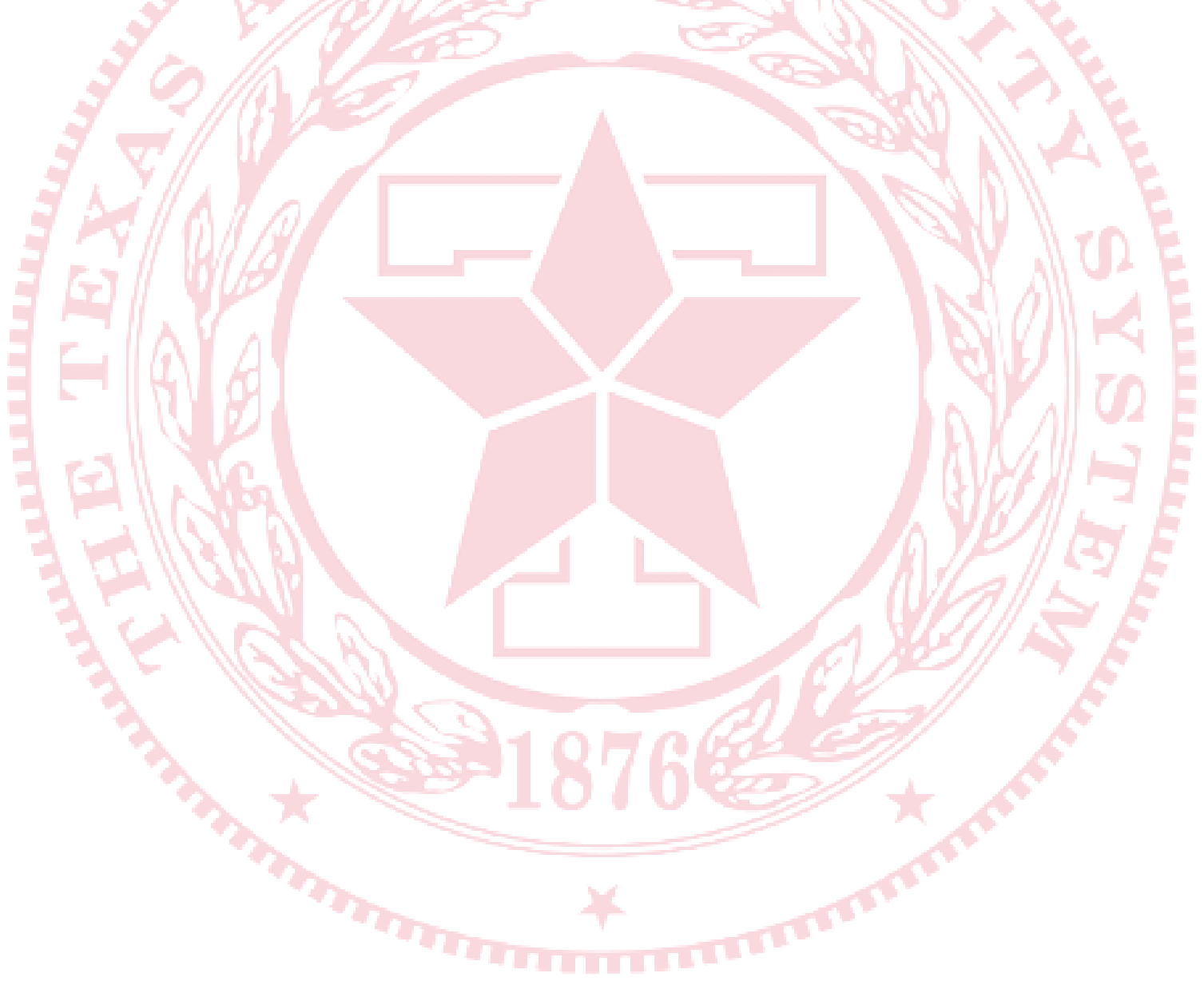




\section{PROJECT OBJECTIVES}

\section{Task 1}

\section{Particle Image Velocimetry (PIV) Measurement of Multiphase during Air Ingress:}

We will measure the temperature distribution and concentration profile of the two species will be investigated using state-of-the-art particle image velocimetry (PIV) and laser induced fluorescence (LIF) techniques. Experiments will be performed in a specially designed small test facility that will allow us to measure the velocity and temperature during stratifications and inflow/outflow behavior through the broken duct. Hot-wire anemometers will also be inserted into the duct and the tanks to obtain local velocity values. These experiments will provide new full-field data of temperature, concentration and velocity components with a high degree of spatial and temporal resolution. The data will allow us to better understand the fundamental flow features and develop improved models.

Task 2

\section{Computational Fluid Dynamics (CFD) Analysis of Air Ingress Phenomena:}

We will construct advanced CFD models that incorporate the multiple physical phenomena associated with air ingress including as molecular diffusion, natural circulation and buoyancy. Modeling of convection is important owing to its potential to contribute to severe fuel and core oxidation. Turbulence modeling will also be employed under the various flow regimes of laminar mixed conditions. We will also utilize our developed large eddy simulation capabilities to capture the multiscales of flow structure. The measured data will provide a unique framework to validate the results of these CFD modeling efforts. 


\section{NOMENCLATURE}

$\mathrm{d}_{\mathrm{P}} \quad$ Particle Diameter $(\mu \mathrm{m})$

$\mathrm{D}_{\mathrm{SF} 6-\mathrm{He}}$ Diffusion Coefficient of $\mathrm{SF}_{6}-\mathrm{He}\left(\mathrm{m}^{2} / \mathrm{s}\right)$

dt Change in Time (s)

$\mathrm{dx} \quad$ Change in Position (m)

Fr Froude Number

g Gravity Term $\left(\mathrm{m} / \mathrm{s}^{2}\right)$

g' Reduced Gravity Term $\left(\mathrm{m} / \mathrm{s}^{2}\right)$

$\mathrm{H} \quad$ Hot Duct Diameter (m)

$\mathrm{L}$ Diffusion Length (m)

$\mathrm{M}_{\mathrm{SF6}-\mathrm{He}}$ Molecular Weight for the Binary Species $(\mathrm{kg} / \mathrm{kmol})$

$\Omega_{\mathrm{D}} \quad$ Diffusion Collision Integral

$\mathrm{P} \quad$ Pressure (atm)

$\rho$ Density of Fluid $\left(\mathrm{kg} / \mathrm{m}^{3}\right)$

$\rho_{\text {Heavy Density of Dense Fluid }\left(\mathrm{kg} / \mathrm{m}^{3}\right)}$

$\rho_{\text {Light }}$ Density of Less Dense Fluid $\left(\mathrm{kg} / \mathrm{m}^{3}\right)$

$\rho_{\mathrm{P}} \quad$ Density of Seeding Particle $\left(\mathrm{kg} / \mathrm{m}^{3}\right)$

Ri Richardson Number

$\sigma_{\mathrm{SF} 6-\mathrm{He}}$ Entropy Generation (Angstrom)

$\mathrm{T}$ Temperature (K)

$\mathrm{T}_{\text {Diff }}$ Diffusion Time Scale (s)

$\mu \quad$ Viscosity ( $\left.\mathrm{Pa}^{*} \mathrm{~s}\right)$

$\mathrm{u} \quad$ Discharge Velocity $(\mathrm{m} / \mathrm{s})$

$\mathrm{u}_{\text {Heavy }}$ Flow Front Velocity of Heavy Density Fluid (m/s)

$\mathrm{u}_{\text {Light }}$ Flow Front Velocity of Light Density Fluid (m/s)

$\mathrm{U}_{\mathrm{g}} \quad$ Gravitational Velocity $(\mathrm{m} / \mathrm{s})$

$V_{\text {Diff }}$ Diffusion Velocity $(\mathrm{m} / \mathrm{s})$

Subscipts

g Gravitational

m Model

p Prototype 


\section{CHAPTER I}

\section{INTRODUCTION}

A new program for future nuclear energy systems, Generation IV, has been created in effort to providenext-generation technologies that will compete in all markets with the most cost-effective technologies expected to be available over the next three decades [1]. This program creates advantages which include reduced capital cost, enhanced nuclear safety, minimal generation of nuclear waste, and further reduction of the risk of weapons materials proliferation. One of the six reactor technologies considered under this program is the Very High Temperature Reactor (VHTR).

VHTRs are a part of the Next Generation Nuclear Plant (NGNP) or Generation IV reactors. The reactor core technology will either be a prismatic block or a pebble bed concept [1] with the cores composed of some type of fuel graphite cladding. The VHTR uses helium as coolant to produce core outlet temperatures in the range of $700-900^{\circ} \mathrm{C}$. These higher temperatures generate higher power conversion efficiencies and provide high quality process heat for chemical processes, including hydrogen production. A level of passive safety is built into all the VHTR's conceptual designs for the next generation nuclear reactors [1]. Passive safety includes safety components which do not require active controller operational intervention to avoid accidents in the event of malfunction. Passive safety may rely on pressure differentials, gravity, natural convection, or the natural response of materials to high temperatures. Past studies have shown that densitygradient dominated stratified flow is an inherent characteristic of passive systems in advanced reactors [2] thus enabling VHTR's to be highly susceptible to this phenomenon.

In the VHTR, air ingress following a loss of coolant accident (LOCA) has been classified as being potentially one of the most severe accidents that can occur [2]. Air ingress occurs when a pipe connecting the reactor vessel and power conversion unit breaks and external air is allowed to enter the reactor vessel from the surrounding reactor cavity. The most catastrophic of these events occurs when there is a double ended guillotine break in the hot duct between the pressure vessel and the power conversion unit [2-4].

The double ended guillotine break leading to a gravity driven air ingress has been identified as a low probability yet high consequence event for VHTR. The lower plenum of the VHTR contains the core support structure and is composed of graphite $[2,3]$. During an air ingress event, oxidation of the graphite structure under high temperature conditions could degrade the integrity of the core support structure. Following this large 
break, air from the reactor containment enters the lower plenum via two mechanisms: diffusion or density driven stratified flow. The large difference in time scales, and hence reaction time, between the mechanisms leads to the need to perform high fidelity experimental and numerical studies to investigate the dominant the air ingress mechanism. A scaled small test facility has been designed and built that allows the acquisition of velocity measurements during stratification and inflow/outflow behavior through a broken duct. Non-intrusive Particle Image Velocimetry (PIV) measurement techniques provide full-field velocity measurements, and concentration profiles of the two species. These experiments provide new high fidelity full-field data of velocities and concentrations with high spatial and temporal resolution. The data allows for fuller concentrations with high spatial and temporal resolution. The data will allow for better understanding of the fundamental flow features, the development of improved models, and possible mitigation strategies in such a scenario.

\subsection{Very High Temperature Reactors}

The VHTR is one of the proposed reactor designs to play a role in future power generation. This reactor is one of six new reactor designs for the Generation IV reactor concepts. The main objective of the VHTR is cogeneration of electricity and hydrogen, as well as to other process heat applications. The major added benefits of the VHTR concept over previous reactors are higher thermal efficiency, hydrogen production, process heat applications, and high degree of passive safety [2]. The general schematic of the VHTR design is seen in Fig. 1. [1]. 


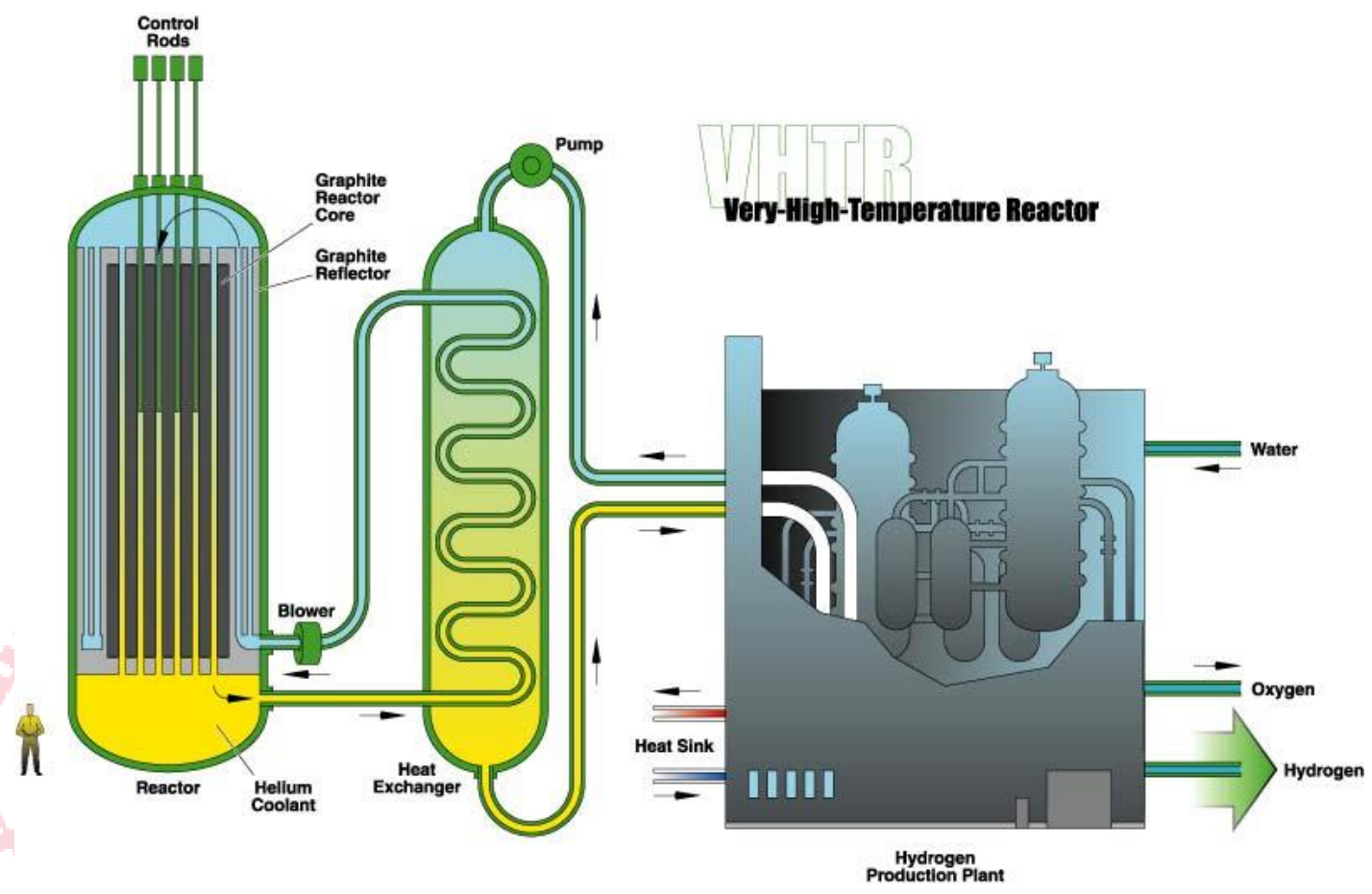

Figure 1: Department of Energy's Reference VHTR Schematic [1]

The basic technology for the VHTR has been well established in former High Temperature Gas Reactor (HTGR) plants, such as Dragon, Peach Bottom, and Fort St Vrain and is being advanced in concepts such as the Gas Turbine-Modular Helium Reactor (GT-MHR) and Pebble Bed Modular Reactor (PBMR) [1]. The VHTR is a helium gas-cooled, graphite-moderated, thermal neutron spectrum reactor with a core outlet temperature greater than $950^{\circ} \mathrm{C}$ [5]. These characteristics make the VHTR sufficient to support production of hydrogen by thermo-chemical processes. The preliminary reactor design is a 600MWth core connected to a steam generator to deliver process heat [2]. This specific thermal power level is set to allow passive decay heat removal.

There are two configurations for the VHTR core: prismatic block or pebble-bed core. The main difference between the configurations is the geometry of the fuel. The main interest of this paper is the prismatic block core configuration. The prismatic core consists of an inner reflector region surrounded by an annulus of fuel blocks which is in turn surrounded by an annulus of outer reflector elements [6]. The basic fuel concept for the VHTR is TRISO coated particles which combined create compacts that fit into the 
fuel blocks. The fuel blocks are composed of hexagonal columns of graphite with circular holes coolant that run the full length of the column.

\subsection{Air Ingress Accident Scenario}

Prior literature pertaining to the air ingress accident scenario is initiated with a pipe break $[2-6,7,8]$. Immediately thereafter, depressurization begins and the hot helium coolant from the reactorvessel escapes. During this process, the helium mixes with the air in the external reactor cavity. Depressurization ceases and air ingress occurs when the pressure in the reactor vessel is equal to the pressure in the containment.

Initial studies focused on molecular diffusion as the primary ingress mechanism with a time scale of around 150 hours [2]. However, recent studies have shown that assuming molecular diffusion as the driving factor in air ingress is physically incorrect. Instead, the primary mechanism for air ingress is shown to be a gravity driven process that occurs due to the large density difference between the internal helium coolant and the external helium-air mixture following a break. The different densities result in a gravity driven flow between the cooler, higher density helium-air mixture in the containment and the hotter, lower density helium present in the reactor vessel. A counter-current exchange flow similar to that modeled by Benjamin's equation [1,9] occurs and the time scale for helium-air mixture to penetrate the lower plenum is expected to be less than 10 seconds depending on break location. The main difference in this presented research case from the previous Benjamin study [9] is that the duct is cylindrical rather than rectangular. Further differentiation of this work from previous air-ingress studies is the presence of a co-annular duct at the break. This duct used in this study is geometrically scaled to model the General Atomics Gas Turbine-Modular Helium Reactor (GT-MHR) [2]. As the gravity driven flow enters the lower plenum region of the vessel, the helium-air mixture begins to heat up at which point natural convection is thought to take place as the now heated mixture begins to rise and drive cooler gasses down the walls of the reactor vessel and out through the cool duct.

Because of the differing time scales of interaction, the primary air ingress mechanism, whether it is dominated by diffusion or density driven stratified flow needs to be verified. Air ingress may result in the oxidation of in-core graphite structures and fuel. Although the amount of oxygen present in the containment is unlikely to cause oxidation to the point of collapse, superficial oxidation of support structures and core materials may result in significant dust generation and resulting fission product release as flows entrain ash particles off the graphite surfaces. By understanding the full cycle of the air ingress scenario through careful experimentation, mitigation strategies may be developed for such an accident. 


\section{CHAPTER II}

\section{LITERATURE SURVEY}

\subsection{Air Ingress Investigations}

To obtain a thorough understanding of the physical phenomena that occurs during a LOCA of a VHTR and for the air ingress scenario as a whole a survey of literature needed to be conducted. The methods and results of various air ingress accident scenario simulations and experiments for LOCA in VHTRs are presented in the following section.

Numerical studies were conducted by Idaho National Laboratory (INL) on Duncan and Toor's [10] two bulb studies using GAMMA and CFX [4]. Duncan and Toor's two bulb studies consist of two bulbs connected by a small diameter pipe of 2.08 $\mathrm{mm}$. One bulb is filled with a heavier gas, $\mathrm{CO}_{2}$ (simulating the ingress of air into the reactor), and the other with a lighter gas, $\mathrm{H}_{2}$ (simulating the reactor coolant). The bulbs are closed to one another prior to the start of the experiment. In this numerical study the exact dimensions of the experimental equipment were used. The findings reveal that the small pipe molecular diffusion is a main phenomenon for gas transport. Findings show that diffusion is a slow process. Even after 200 seconds the gas concentrations of the lighter gas bulb and the heavier gas bulb are not changed [4]. For the second numerical study conducted in 2009 at INL [4], the same dimensions were used as in the experiment and the diffusion two bulb analyses except for the diameter of the capillary tube. The capillary tube was changed to a diameter of $16 \mathrm{~mm}$. Figure 2 shows the simulation after 30 seconds and depicts the heavier gas $\mathrm{CO}_{2}$ flows to the bottom and the lighter gas hydrogen is on top of the heavier gas. This indicates that the density-gradient-driven stratified flow is a dominant phenomenon for the gas species in a larger size pipe of 16 $\mathrm{mm}$ [4]. This reveals the large time difference in the two ingress mechanisms and indicates that further investigation is needed when a LOCA occurs in a VHTR. 
$\mathrm{CO} 2$ at STP. Molar Fraction

(Contour 1)

- 3. $839 \mathrm{e}-01$

$-3.698 \mathrm{e}-01$

$-3.557 \mathrm{e}-01$

$-3.417 \mathrm{e}-01$

$-3.276 \mathrm{e}-01$

$-3.136 \mathrm{e}-01$

$-2.995 \mathrm{e}-01$

$-2.855 e-01$

$-2.714 \mathrm{e}-01$

$-2.574 \mathrm{e}-01$

$-2.433 e-01$

$-2.293 e-01$

$-2.152 \mathrm{e}-01$

$-2.011 \mathrm{e}-01$

$-1.871 \mathrm{e}-01$

$-1.730 \mathrm{e}-01$

$-1.590 e-01$

$-1.449 \mathrm{e}-01$

$-1.309 \mathrm{e}-01$

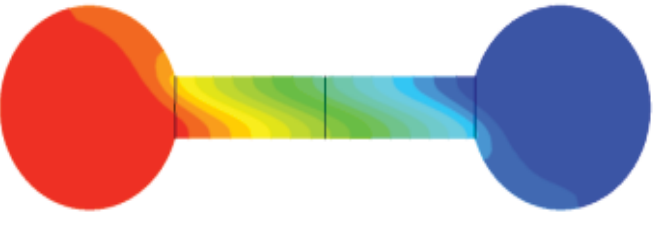

$1,168 \mathrm{e}-01$

Figure 2: CFX Results of Two-Bulb Simulation with 16-mm Pipe [4]

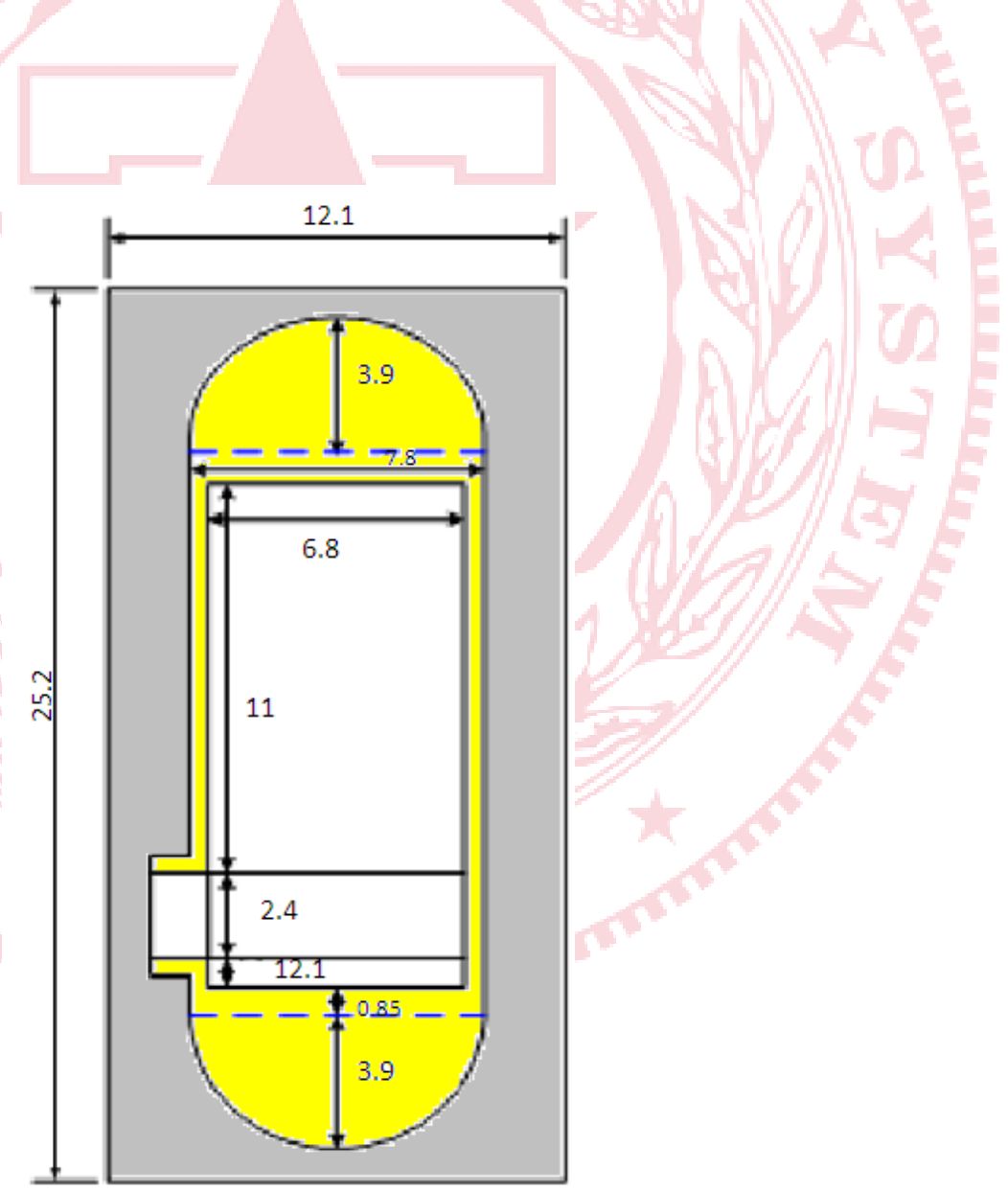

Figure 3: Simplified 2-D Geometry of GT-MHR for Stratified Flow Simulation [5] 
To further the investigation of the air ingress mechanism, INL performed a preliminary numerical study on the stratified flow phenomena in the VHTR LOCA [5]. This study was investigated using FLUENT 6.3 using a 2-D model of GT-MHR 600MWt reactor reference geometry. This 2-D model was constructed with 5 major zones as seen in Fig. 3 with the size of the reactor cavity not being taken into account.

The investigation revealed that air ingresses rapidly into the reactor core with counter-current stratified flow shape [5]. In this calculation, it only took 60 seconds for the air to fill up the lower plenum and to stabilize. This time scale is instantaneously small compared to the whole air ingress time frame that was found for the diffusion ingress mechanism ( $150 \mathrm{hrs})$ [5].

In efforts to estimate the consequences of the stratified flow assumption as the air ingress mechanism, INL performed another numerical investigation. This investigation examines the whole air ingress scenario using both Fluent and GAMMA codes [2]. The main purpose for this investigation was to understand the stratified flow effect as air ingresses into the reactor and to find the onset of natural convection. The GT-MHR was used as the reference geometry and the Fluent simulations reveal that natural convection was initiated 160 seconds after stratified flow was started and also the whole reactor vessel was filled with air after 4 minutes [2]. This accelerated onset of natural convection leads to much faster oxidation in the graphite structures. The conclusion (new assumption-stratified flow) was that air ingress is a much more severe than previously thought and the previous assumption on air-ingress accident will lead to the underestimation on their consequences. It is therefore recommended by INL that the original air-ingress scenario based on molecular diffusion be replaced with the new assumption considering stratified flow.

Studies on density-gradient-driven stratified flow in advanced reactor systems has been the subject of active research for over a decade because density-gradient dominated stratified flow is an inherent characteristic of passive systems used in Light Water Reactors (LWR) [7]. Liou, 2007 performed density driven stratified flow experiments using water as the working fluid instead of helium. In one experiment he used air, oil, and water to develop a visualization of the role density plays on varying fluids in a pipe. The LWR is conceptually identical and directly applicable to the phenomenological behavior that occurs in the NGNP. The governing equations from this experiment are identical to the ones used in the air ingress event of a VHTR [7].

In 2010, Oh and Kim [8] conducted experiments to investigate density driven stratified flow during a LOCA of a VHTR. The experiments were conducted in two acrylic tanks connected by a pipe using brine and water as the working fluids. The fluids were separated by a large valve to simulate the pipe break and the facility was scaled 
down model of a GT-MHR as the reference reactor. The isothermal experiments had two objectives:

1) to understand stratified flow phenomena in the VHTR and

2) to provide experimental data for validating computer codes.

The experiment shows clear stratified flow between the heavy and light fluids. Also, Benjamin's model provides a good prediction for flow front speed for internal stratified flow. This model was used as a comparison to the experimental data in which less than $10 \%$ error was found [8].

All of these investigations provide insight into the air ingress scenario. Without knowing what has been done in the past, nothing can be improved in the future.

\subsection{This Work}

In this work, experiments were performed to investigate the dominant air ingress mechanism during a LOCA. A small scaled test facility was designed and built to allow the acquisition of velocity measurements during stratification and inflow/outflow behavior through the broken duct. Investigations include two shadowgraphy investigations with different pipe break locations and one Particle Image Velocimetry (PIV) investigation. 


\section{CHAPTER III}

\section{THEORY}

Density driven currents, or often called gravity driven currents, are induced by density variations due to a difference in temperature, presence of a dispersed solid phase, or heavier dense gas. Lock exchange flows are a class of density currents in which surface tension can be neglected and counter current flows are produced. These are simple flow configurations, which may, however, result in very complex flows characterized by physical processes such as the emergence of Kelvin-Helmholtz-like instabilities, the formation of lobes and clefts at the front leading edge, etc [11].

Lock exchange flows consists of two fluids of different densities initially separated by a gate. When the gate is removed, differences in the hydrostatic pressure cause the denser fluid to flow in one direction along the bottom boundary of the tank, while the lighter fluid flows in the opposite direction along the top boundary of the tank [12]. A basic configuration of the flow is shown in Fig. 4.

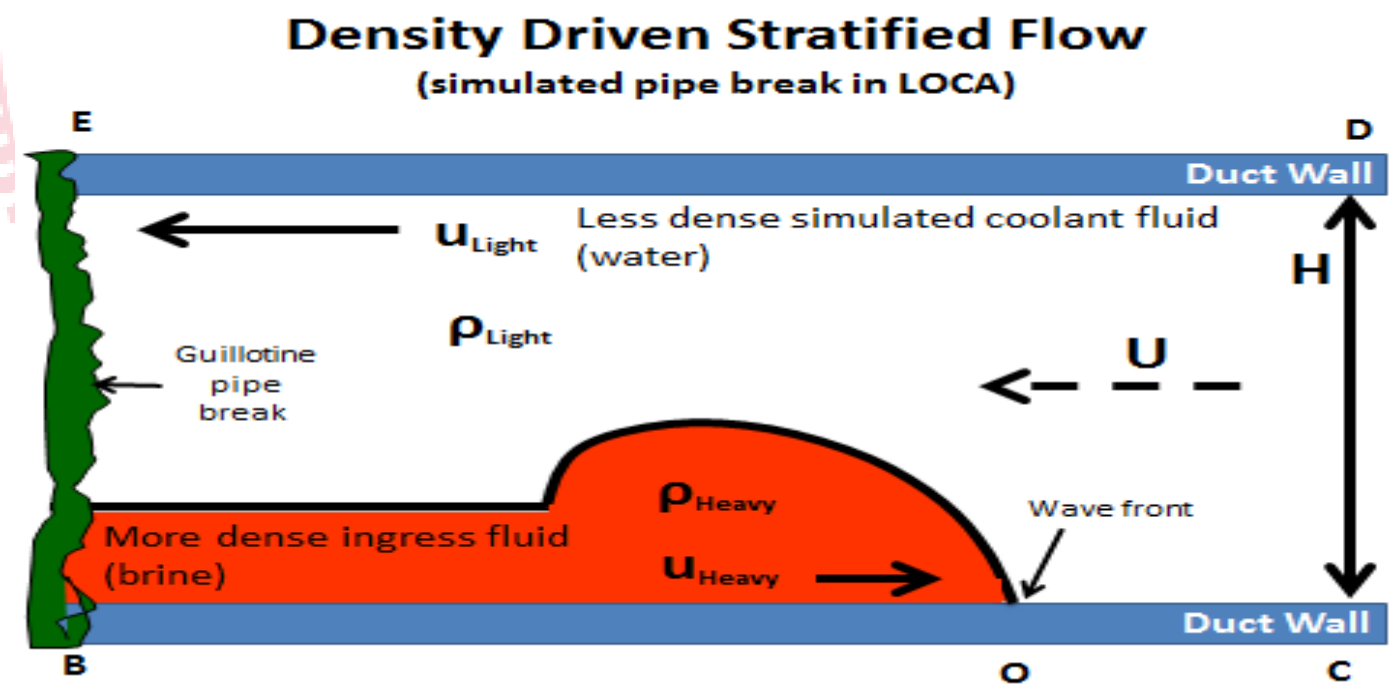

Figure 4: A Schematic Diagram of an Idealized Gravity Current 
The rectangular block was the first agreed upon assumption of many investigators of lock exchange flow. The major assumption in the approximation is that it follows inviscid fluid theory. By equating decreasing potential energy to increasing kinetic energy, the following result is obtained [13]:

$$
\frac{\mathrm{U}_{\text {Heavy }}}{\sqrt{\left(\mathrm{g}^{\prime} \mathrm{H}\right)}}=0.5
$$

Experiments revealed a very close value to be 0.44 .

The inviscid fluid theory provides a useful approximation to the behavior of a gravity current front. This theory assumes no viscous forces are present. Benjamin [9] analyzed the front of a frictionless gravity current. The Benjamin model was used in this paper to analyze the flow front.

Lock exchange flow progresses in three stages [14]:

1) After the initial collapse of fluid when the gate is removed there is an adjustment phase in which the front advances at constant speed.

2) The second stage is self- similar flow in which the gravity current is collapsing. The current depth is decreasing with time.

3) The third stage comes in effect if viscous effects become dominant.

Most of the mixing within the density current occurs in the front, or often called the head of the current. The mixing has two major effects on the transport of the dense fluid [15]:

1. It locally increases the internal near bed velocity of the flow with respect to the front propagation rate.

2. It increases the total amount of fluid transported for known current characteristics.

There are two dominant types of instabilities that are responsible for mixing that occur in density currents, billows and clefts and lobes. Billows are an instability that rolls up in the region of velocity shear above the front of the dense fluid. A certain type of billow that is explored is the Kelvin Helmholtz instability. This instability is formed at the interface between two fluids of different density moving relative to each other. The complex shifting pattern of clefts and lobes are formed by the influence of the ground or bottom of the tube on the lower part of the edge [14]. 


\section{CHAPTER IV}

\section{SCALING ANALYSIS}

To validate the fact that the scaled down laboratory scale experimental apparatus effectively simulates conditions expected in the VHTR, this section discusses the scaling analysis for air ingress via density driven stratified flow phenomenon in the experimental simulation of the VHTR during a LOCA. This section identifies the respective dimensionless groups and similarity criteria used to describe this phenomenon.

Scaling analysis was performed for the density driven stratified flow phenomena in a VHTR. Commonly used dimensionless numbers to characterize stratified flow are Richardson and Froude numbers under the Boussinesq approximation. The essence of the Boussinesq approximation is that the difference in inertia is negligible but gravity is sufficiently strong to make the specific weight appreciably different between the two fluids.

In the scaling analysis, the flow front velocity of the light and heavy fluids are assumed to closely follow Benjamin's equation, Eq. (2) and (3), based on previous studies with a single cylindrical pipe [9]. The dimensionless numbers are matched in the model and prototype with a reduced gravity term, Eq. (4) and are set to unity as seen in Eq. (5) and Eq. (6).

$$
\begin{aligned}
& \mathrm{u}_{\text {Heavy }}=0.44 \sqrt{\mathrm{g}^{\prime} \mathrm{H}} \\
& \mathrm{u}_{\text {Light }}=0.44 \sqrt{\mathrm{gH}\left(\rho_{\text {Heavy }}-\rho_{\text {Light }}\right)} \\
& \mathrm{g}^{\prime}=\mathrm{g} \frac{\rho_{\text {Heavy }}-\rho_{\text {Light }}}{\rho_{\text {Avg }}} \\
& \frac{\mathrm{Ri}_{\mathrm{m}}}{\mathrm{Ri}_{\mathrm{p}}}=\frac{\mathrm{u}_{\mathrm{p}}{ }^{2} \mathrm{~g}_{\mathrm{m}}^{\prime} \mathrm{h}_{\mathrm{m}}}{\mathrm{u}_{\mathrm{m}}^{2} \mathrm{~g}_{\mathrm{p}}^{\prime} \mathrm{h}_{\mathrm{p}}}=1 \\
& \frac{\mathrm{Fr}_{\mathrm{m}}}{\mathrm{Fr}_{\mathrm{p}}}=\frac{\mathrm{u}_{\mathrm{m}} \sqrt{\mathrm{g}_{\mathrm{p}}^{\prime} \mathrm{h}_{\mathrm{p}}}}{\mathrm{u}_{\mathrm{p}} \sqrt{\mathrm{g}_{\mathrm{m}}^{\prime} \mathrm{h}_{\mathrm{m}}}}=1
\end{aligned}
$$

As seen in the previous equations, the Froude number $\left(\mathrm{Fr}_{\mathrm{m}} / \mathrm{Fr}_{\mathrm{p}}\right)$ is the ratio of inertial forces to gravitational forces and the Richardson number $\left(\mathrm{Ri}_{\mathrm{m}} / \mathrm{Ri}_{\mathrm{p}}\right)$ is the ratio of potential energy to kinetic energy. Both are highly dependent on the density ratio and the 
characteristic length scale $(\mathrm{H})$. With the scaled down experimental test facility and the resulting scaled down characteristic length, there was a need to adjust the density ratio to obtain the necessary Froude and Richardson numbers that represent reactor conditions during a LOCA to provide representative results. To accomplish this, different fluids were used to adjust the density ratio to obtain the same dimensionless numbers as in the actual reactor. The density ratio for the actual reactor is 0.14 and to obtain this same ratio a variation in concentrations for the fluids are used. These fluids are found in Table 1.

Table 1: Fluids Used for Reactor Similarity

\begin{tabular}{|lllll|}
\hline $\begin{array}{l}\text { Fluids } \\
\text { Used }\end{array}$ & $\begin{array}{l}\text { Froude } \\
\text { Number }\end{array}$ & $\begin{array}{l}\text { Richardson } \\
\text { Number }\end{array}$ & $\begin{array}{l}\text { Density } \\
\text { Ratio } \\
\left(\rho_{\text {light }} / \rho_{\text {heavy }}\right)\end{array}$ & $\begin{array}{l}\text { Flow Front } \\
\text { Velocity Ratio } \\
\left(\mathrm{u}_{\text {heavy }} / \mathrm{u}_{\text {light }}\right)\end{array}$ \\
\hline Helium-Air (Reactor) & 0.33 & 0.68 & 0.14 & 2.69 \\
Water-Brine & 0.43 & 4.89 & 0.88 & 1.06 \\
Helium-SF6 & 0.33 & 0.68 & 0.14 & 2.69 \\
Helium-CO2 & 0.33 & 0.87 & 0.14 & 2.69 \\
\hline
\end{tabular}

Although not all the fluids listed in Table 1 are of the same density ratio and dimensionless numbers, each fluid combination played a specific purpose.

- The air ingress facility was scaled using sulfur hexafluoride $\left(\mathrm{SF}_{6}\right)$ and helium as the working fluids. These two fluids match the dimensionless numbers and density ratio which in turn allows the flow front velocity ratios to be the same that occur in the actual air ingress scenario of the reactor.

- Carbon dioxide and helium will be used as a comparison to the sulfur hexafluoride-helium case with matching Froude number, density ratio, and flow front velocity ratios. Even though the Richardson number was not matched in this case due to the different densities, this case provides a good prediction of flow during the air ingress scenario.

- The brine-water case acts merely as a flow visualization to capture important points prior to first testing and as initial validation to CFD results of the brine-water case. The brine-water case does not match the dimensionless numbers as in the reactor due to the fluids needed for the flow visualization. Prior to the flow visualization experiment, the density of the brine solution and water were measured by a hydrometer and the viscosity measured by a 
rheometer to ensure accurate estimates of the flow front velocity. These dimensionless parameters along with the Reynolds number were used to calculate the flow regime.

From this scaling analysis, a test facility was constructed to faithfully represent the density driven stratified flow phenomenon as seen in Fig. 5. From geometric scaling, this test facility is approximately 1:20 length scale of the actual reactor size.

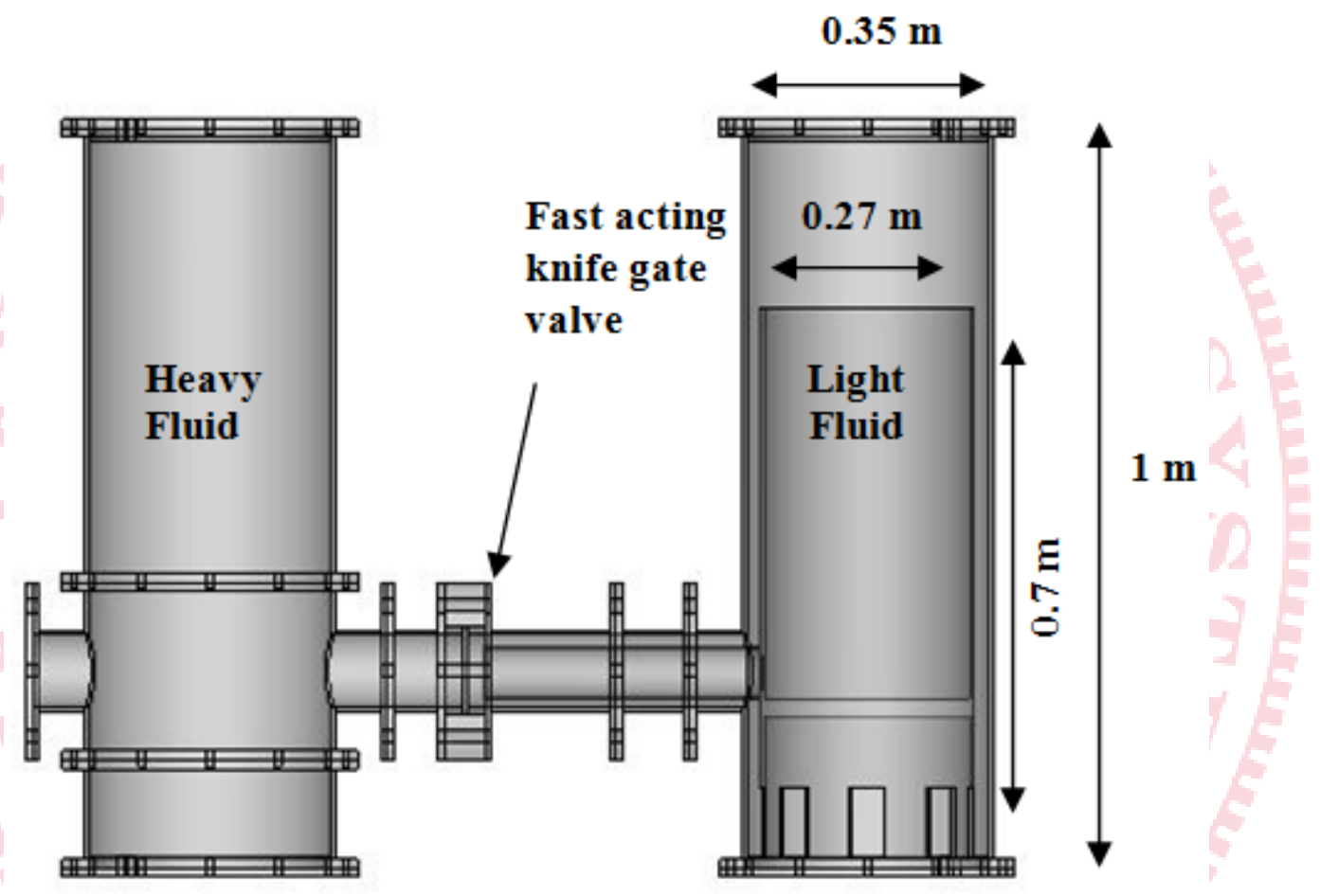

Figure 5: Air Ingress Test Facility 


\section{CHAPTER V}

\section{EXPERIMENTAL APPROACH}

\subsection{Experimental Set-up}

The isothermal stratified flow experiment consists of two acrylic tanks and a horizontal coaxial pipe connecting the two tanks as seen in Fig. 5. The inner pipe has a diameter of $0.066 \mathrm{~m}$ and the outer pipe has a diameter $0.1 \mathrm{~m}$ with a length of $1.0 \mathrm{~m}$. The tanks have a diameter of $0.35 \mathrm{~m}$, and a height of $1.0 \mathrm{~m}$. The heavy fluid tank that simulates the reactor vessel has an inner tank to simulate the reactor core. This inner tank has a diameter of $0.29 \mathrm{~m}$ and a height of $0.7 \mathrm{~m}$.

Both the tanks and the coaxial pipe are made of a transparent acrylic for optical measurements and flow visualization. Along the horizontal coaxial pipe is a sliding knife gate valve (Dezurik Knife Gate Valve, KGC, 5, F1, S1, TDP, S1-CR*CY-PC6, 4V1045) installed to separate the tanks, which can be seen in Fig. 6. Initially, both tanks were filled with fluids having different densities, and the valve was closed. To initiate the experiments, the valve was quickly opened (simulating a guillotine break) with $80 \mathrm{psi}$ compressed air. As a result of the break, a counter-current stratified flow formed in the test-section where the heavy fluid intruded into the light fluid at the bottom of the tank, and the light fluid intruded into the heavy fluids at the top of the tank.

\subsection{Visualization Techniques}

Several visualization techniques were used to analyze the density driven stratified flow front in the simulated air ingress scenario of a VHTR during a LOCA. Among these techniques were Shadowgraphy and Particle Image Velocimetry (PIV).

\subsubsection{Shadowgraphy, Setup, and Flow Measurement}

Initial flow visualization tests were conducted using a brine solution to simulate the heavy helium-air mixture in the reactor containment, and water to simulate the hot helium exiting the reactor vessel. Flow visualizations using shadowgraphy techniques were conducted to investigate the stratified flow that occurs following a pipe break. Shadowgraphy is an optical method that depicts fluid flow patterns made visible by using differences in index-of-refraction in the flow. The fluid is illuminated by a beam of light which bends toward regions of higher refractive index while passing through a transparent material. In our experiment, the light beam used in shadowgraphy is the LEC light source which provides a monochromatic source of light. 

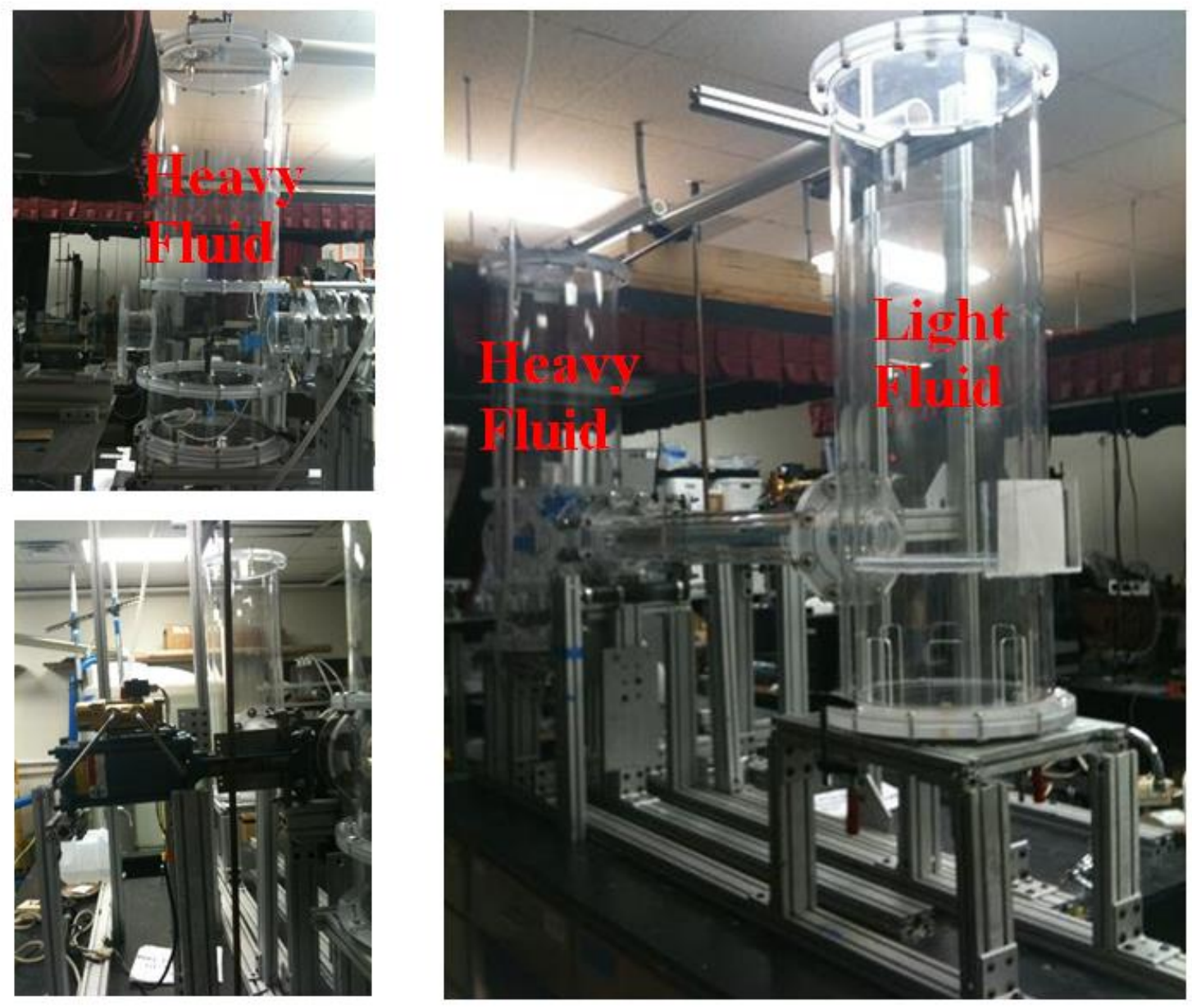

Figure 6: Isothermal Stratified Flow Experimental Setup 


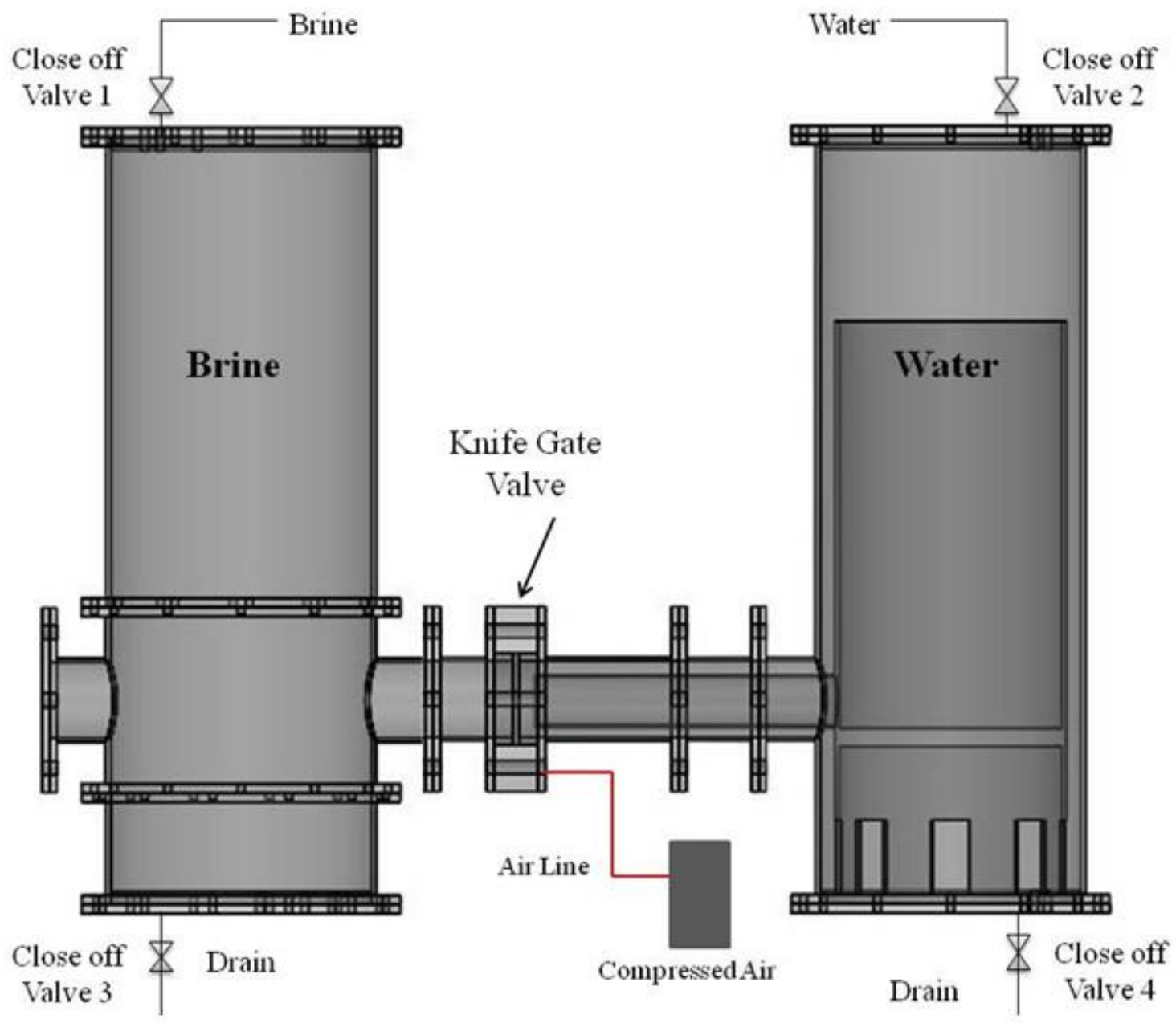

Figure 7: Schematic for the Isothermal Air Ingress Experiment 
Shadowgraphy was used to measured the relative flow front velocity in the pipe as it progresses into the lower plenum. To accomplish this, two fluids, a dyed brine solution and water were used with a fast actuating knife gate valve to simulate the pipe break. High speed cameras captured the flow of the two fluids in the pipe as it entered the lower plenum. Figure 7 shows the schematic for the isothermal air ingress experiment. Initially, the knife gate valve is closed and valves 3 and 4 are closed. The brine solution is mixed prior to experiment and dye is added. The density and viscosity are measured prior to experiment with a hydrometer and viscometer respectively. The water and brine solution is added to respective tanks filling through the feed lines into through valves 1 and 2 . The pneumatic knife gate valve is pressurized with 80 psi of compressed air and is opened by turning on a switch. The flow pattern is captured in the horizontal coaxial pipe by high speed cameras. After the experiment, the power source to the knife gate valve is de-energized and valves 3 and 4 are opened to discharge the fluids to be discarded.

To capture the flow, one to two high speed cameras (number depends on length of pipe) are placed perpendicular to the pipe interrogation region depending on the break location and another camera is placed on top of the tank that symbolizes the reactor vessel to capture flow propagation entering the lower plenum. The break location varies which changes the length of the pipe. This creates a need for different number of cameras to capture the flow front. Figure 8 shows the camera set-up of the flow visualization for the brine-water investigation. The camera specifications used for this investigation are given in Table 2.

Table 2: Camera Specifications

\begin{tabular}{|c|c|c|c|}
\hline & Camera 1 & Camera 2 & Camera 3 \\
\hline Location & $\begin{array}{l}\text { Viewing co-annular } \\
\text { pipe from the front } \\
\text { (blue box) }\end{array}$ & $\begin{array}{l}\text { Viewing co-annular } \\
\text { pipe from the front } \\
\text { (red box) }\end{array}$ & $\begin{array}{l}\text { Viewing reactor vessel } \\
\text { lower plenum from } \\
\text { above }\end{array}$ \\
\hline Make & Vision Research & Phantom & Vision Research \\
\hline Model & v7.3 & Ultima-ATX & V7.3 \\
\hline Max. Resolution & $800 \times 600$ pixels & $1024 \times 1024$ pixels & $800 \times 600$ pixels \\
\hline Frame Rate & 6688 & 2000 & 6688 \\
\hline Optics & \multicolumn{3}{|c|}{ Carl Zeiss Makro-Planar T* $2 / 50$} \\
\hline
\end{tabular}




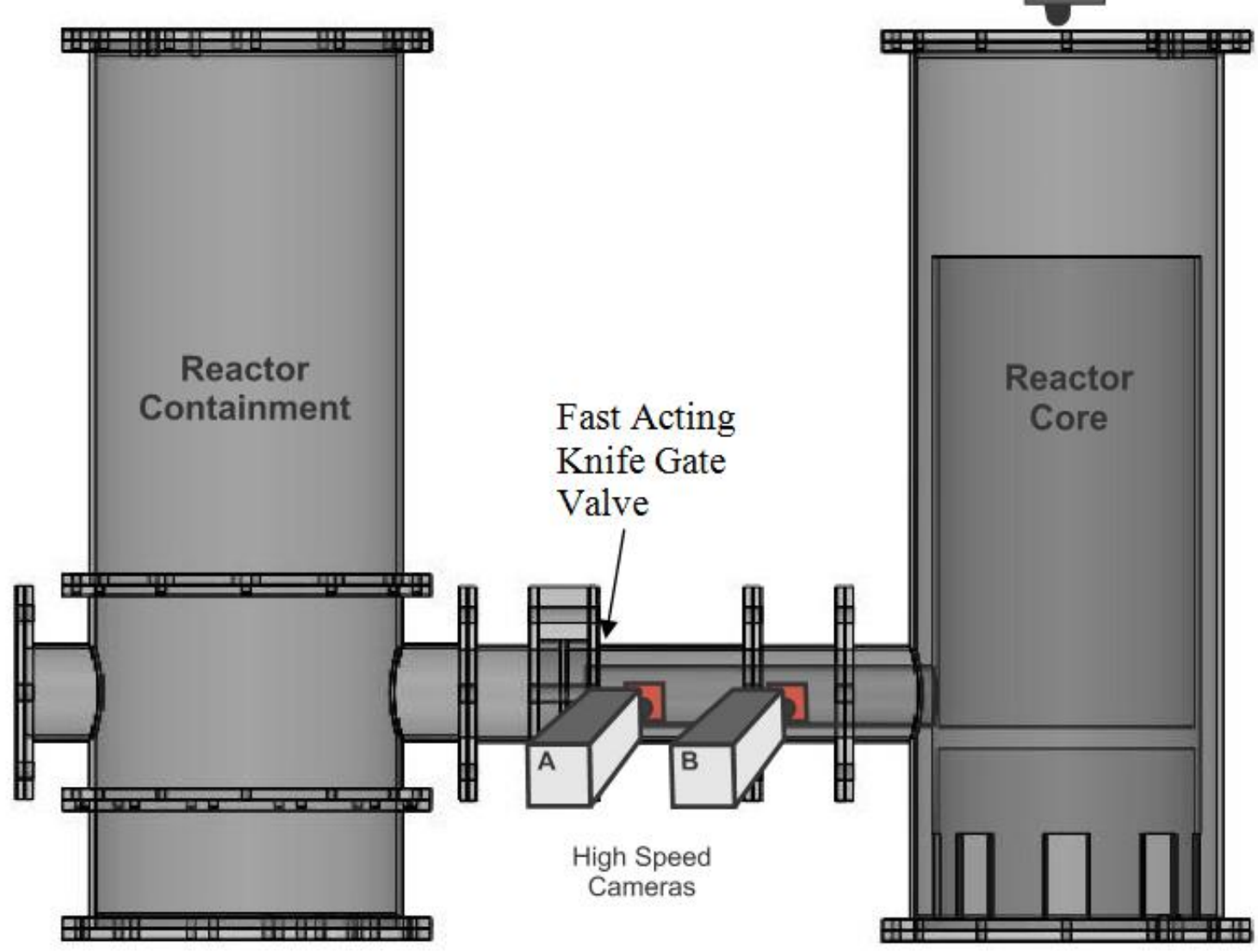

Figure 8: Brine-water Camera Setup for Long Pipe Case

Figure 9 shows the backlight illumination source used for the shadowgraphy in a post brine-water experiment. A flexible 36"x12" Ceelite light emitting capacitor (LEC) provided monochromatic illumination along the length of the pipe and a round 12" LEC was used below the simulated reactor vessel to provide illumination for the tank camera (Camera 3).

Flow front velocity measurements were conducted using one to two high speed cameras at the pipe and spreading rate measurements were conducted using one camera over the simulated reactor tank (light water tank in Fig. 8). Each camera has a known frame rate (Table 2). Prior to the experiment, the distance from the guillotine break along the tube is measured and marked. These distance measurements are used to 
measure the wave front travel as it propagates down the tube. With the use of image processing software, the change in distance of the wave front is correlated with the number of frames to determine the wave front travel time. The comparison of the travel time with the travel distance along the tube provides the wave front velocity.

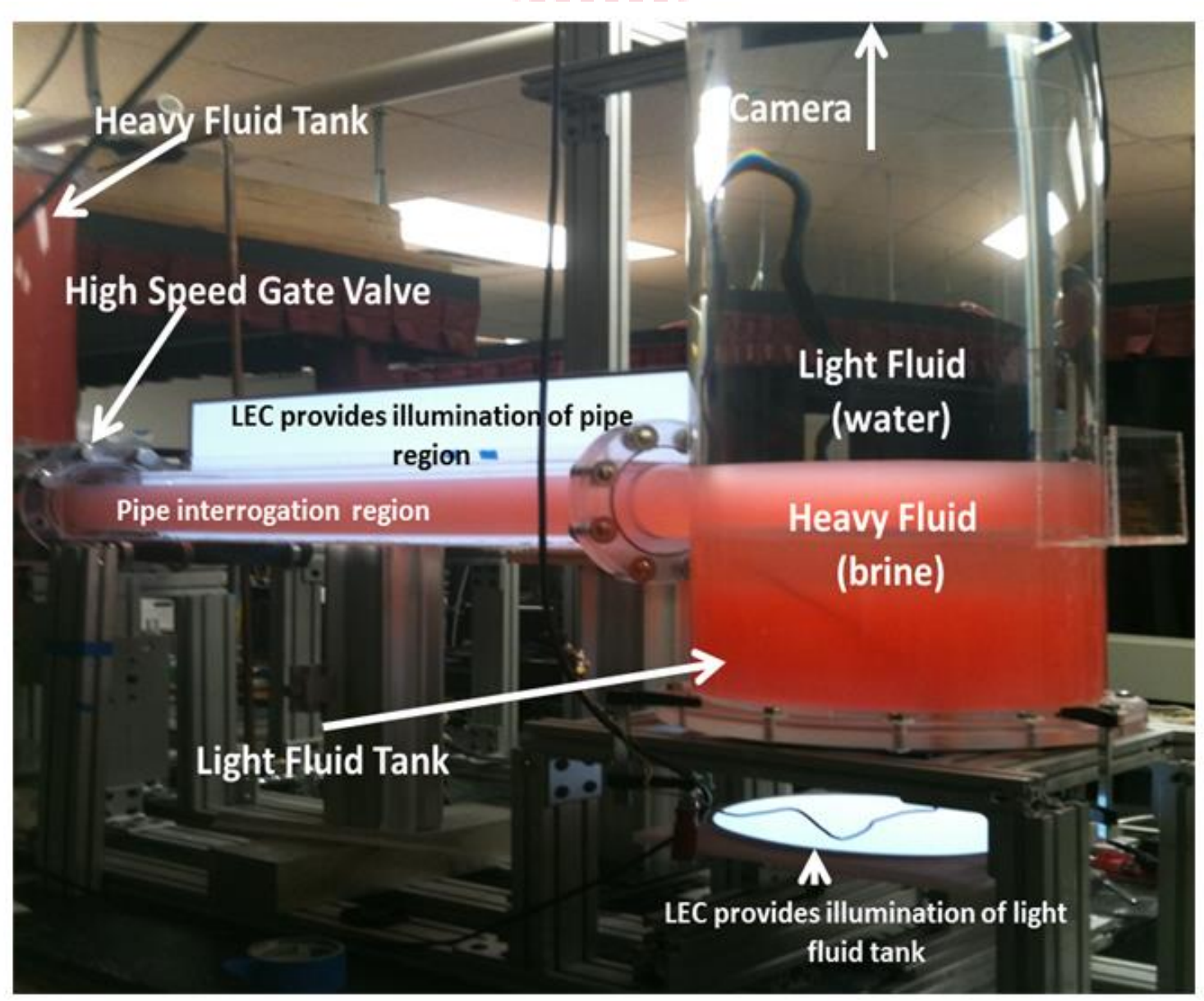

Figure 9: Shadowgraphy in a Post Brine-water Experiment

\subsubsection{Particle Image Velocimetry (PIV) Technique, Setup, and Flow Measurement}

Particle Image Velocimetry (PIV) is a non-intrusive optical measurement technique that provides full-field quantitative and qualitative information of the flow with high spatial and temporal resolution. The measuring principle is based on the fact that instantaneous fluid velocities can be measured by recording the position of images produced by small tracers suspended in the fluid, at successive time increments. 
PIV methods inherently measure the Lagrangian velocities of the tracer particles [16]. The underlying assumption is that these tracer particles closely follow the fluid motion with minimal lag. This assumption holds true for a wide variety of flows of interest, provided that the tracers are small enough and/or their density approaches that of the fluid. To improve measurement accuracy of the flow velocity, PIV needs a high concentration of tracers with the measurement of the "local" fluid velocity being obtained from an average over many tracers contained in a measurement volume.

Experiments were performed in a specially designed small test facility that allowed the measurement of the velocity and the temperature during stratifications and as a result evaluate the inflow/outflow behavior through the broken duct. The experimental setup for PIV investigations of gas-gas scenarios is seen in Fig. 10. The visualization system consisted of particles flow tracers, a high-speed high-resolution camera, a high-power laser, a continuous halogen lamp, mirrors, translational stages, and lenses. These experiments provided full-field data of velocity with a high degree of spatial and temporal resolution. The analysis of the data provided an understanding of the fundamental flow features which could lead to improved CFD models the air ingress

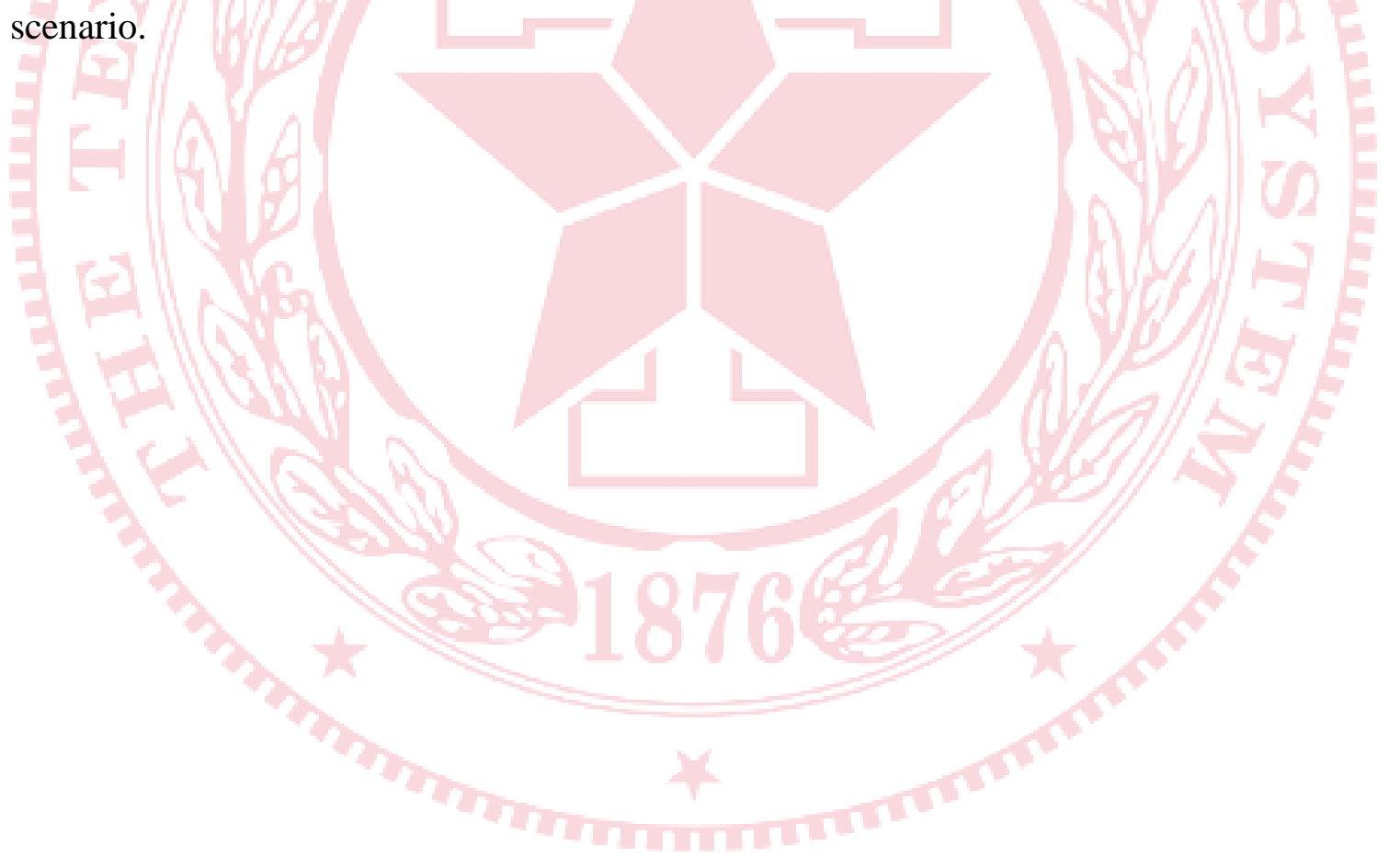




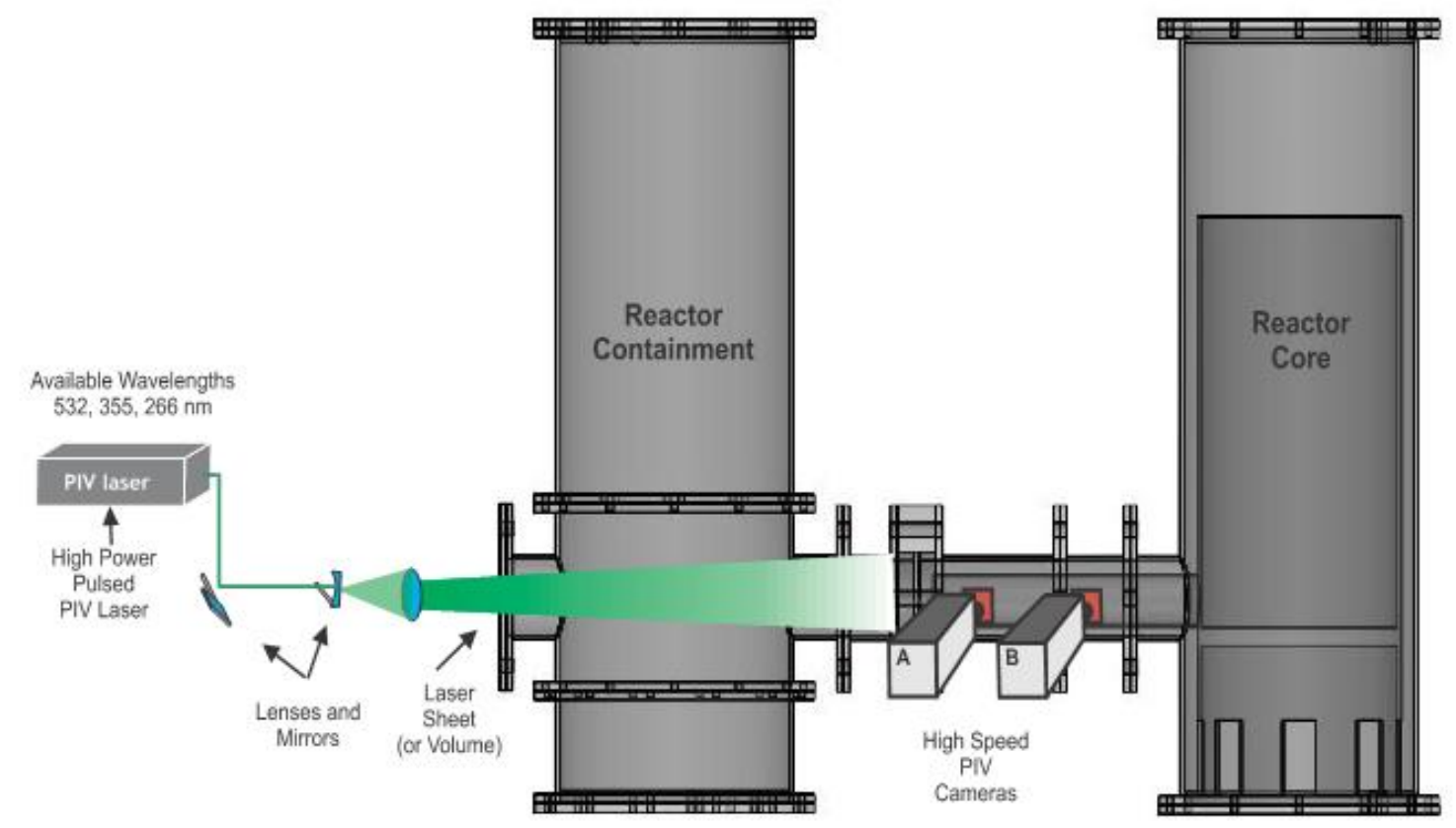

Figure 10: Gas-gas Investigation Camera Setup for Long Pipe Case

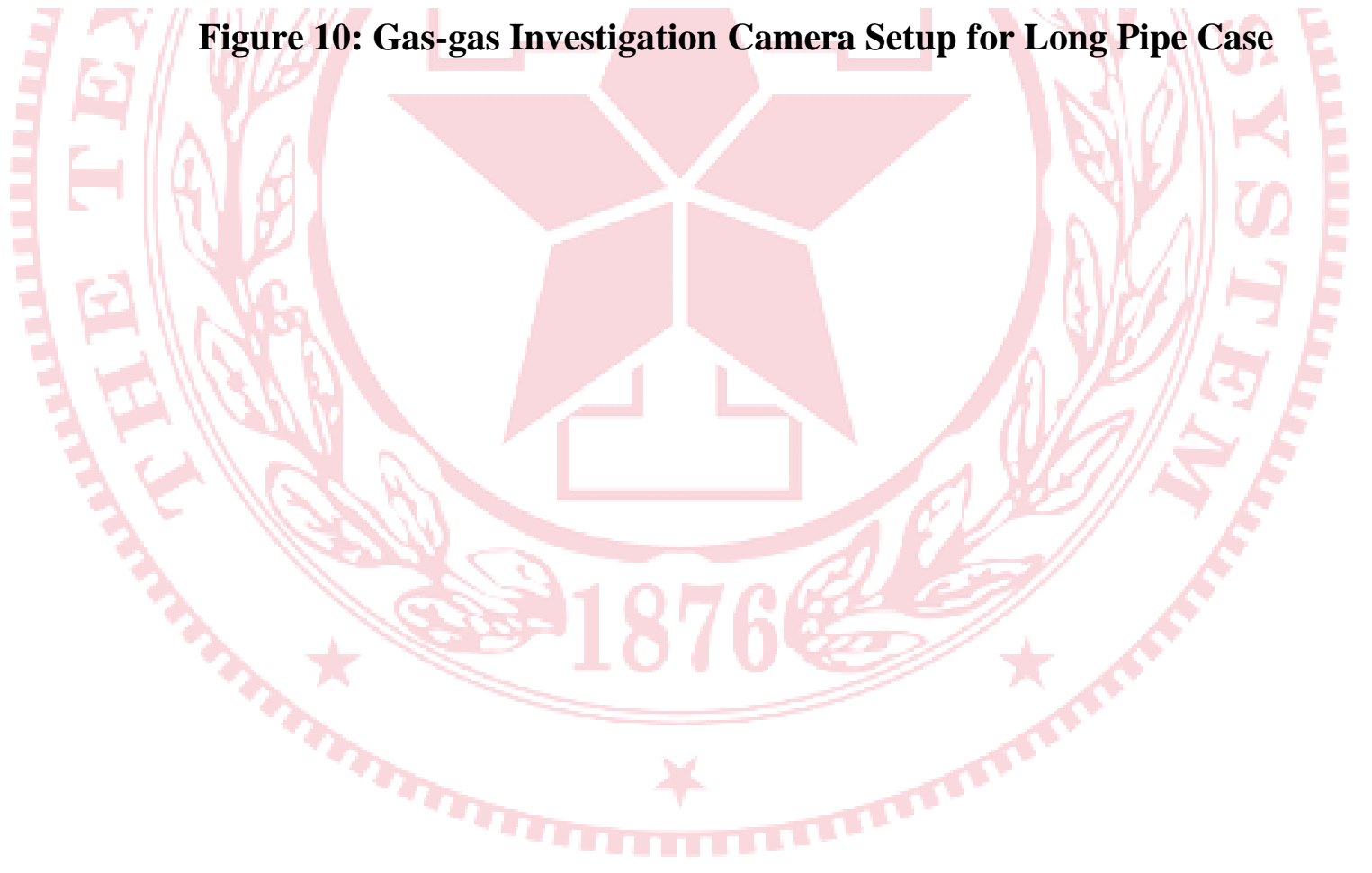




\section{CHAPTER VI}

\section{UNCERTAINTY}

\subsection{Experimental Uncertainty}

This section identifies uncertainties associated with field of view errors in the flow visualization investigations. For both case A and case B, uncertainties arise when measuring flow front velocity. The cameras are lined up to catch the flow after the pipe break and the flow as it propagates down the investigation region of the pipe (these investigation regions are different for both cases and are specified in Figs. 11 and 12). The cameras are aligned to the edge of the pipe and measurements of the flow front occur from the middle of the pipe. Therefore the maximum uncertainty occurs at the edge of the pipe where the flow front is at the widest angle.

Uncertainty measurements were calculated by measuring the angle projected by the field of view and the pipe. This angle was then used to calculate the skewed length from where the flow front measurements are taken place. The maximum uncertainty is calculated using Eq. (7).

$$
\text { Max.Uncert. }=\frac{\text { Skewed Region }}{\text { Investigation Region+Skewed Region }}
$$

Schematics of these uncertainty measurements are seen in Fig. 11 and 12.

Case A was found to have a maximum uncertainty of $4.29 \%$ due to the angle of $14.7^{\circ}$ skewed angle. Case B utilized two cameras which both have their own uncertainty associated with the angle. Case B was found to have a maximum uncertainty of $1.8 \%$ and $1.89 \%$ respectively of the two cameras due to the skewed angles of the two cameras used. These two uncertainties for Case B were combined to get the maximum uncertainty of $3.69 \%$. All of the uncertainties were propagated to the velocity calculations. 


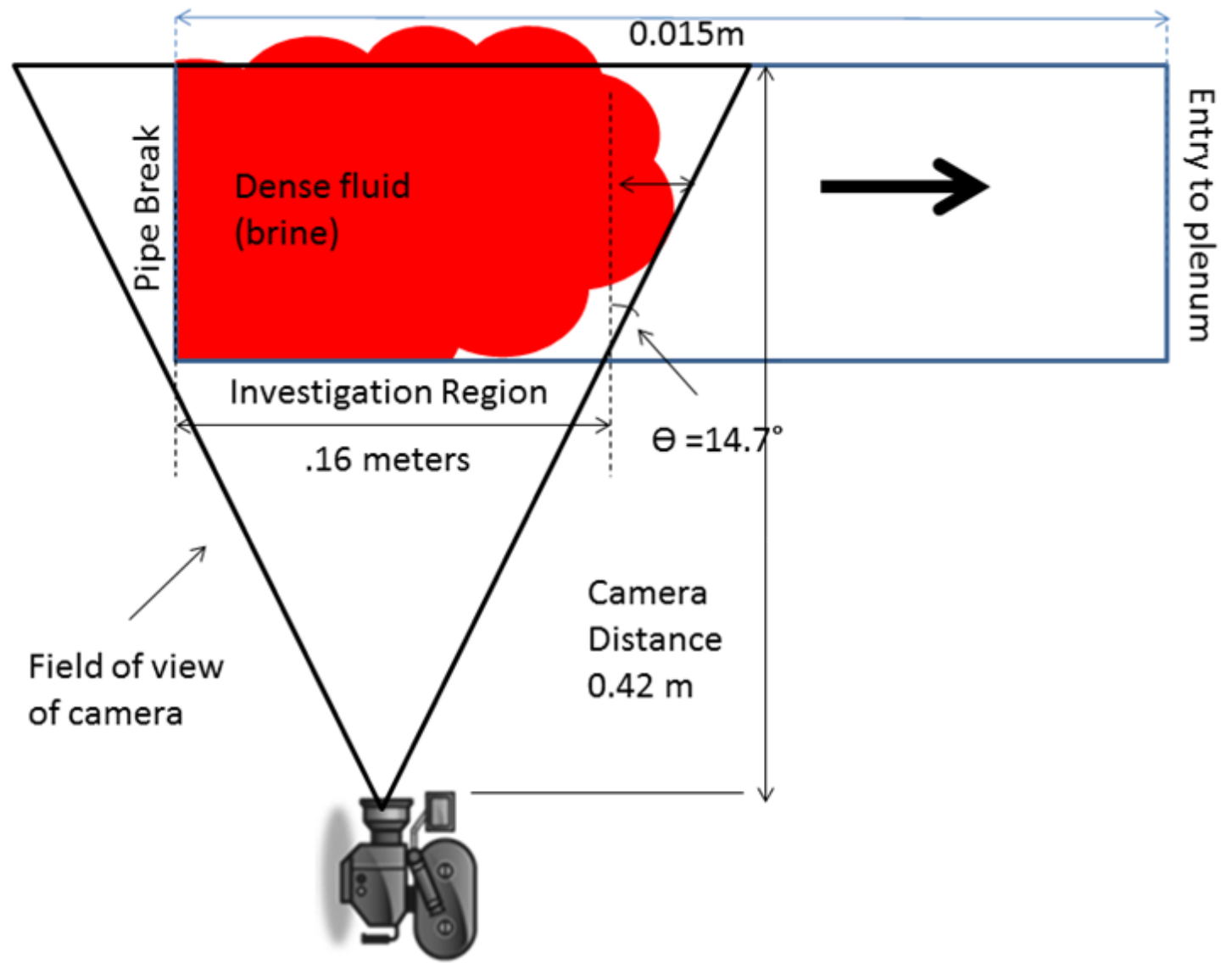

Figure 11: Uncertainty Associated with the Field of View in Case A 


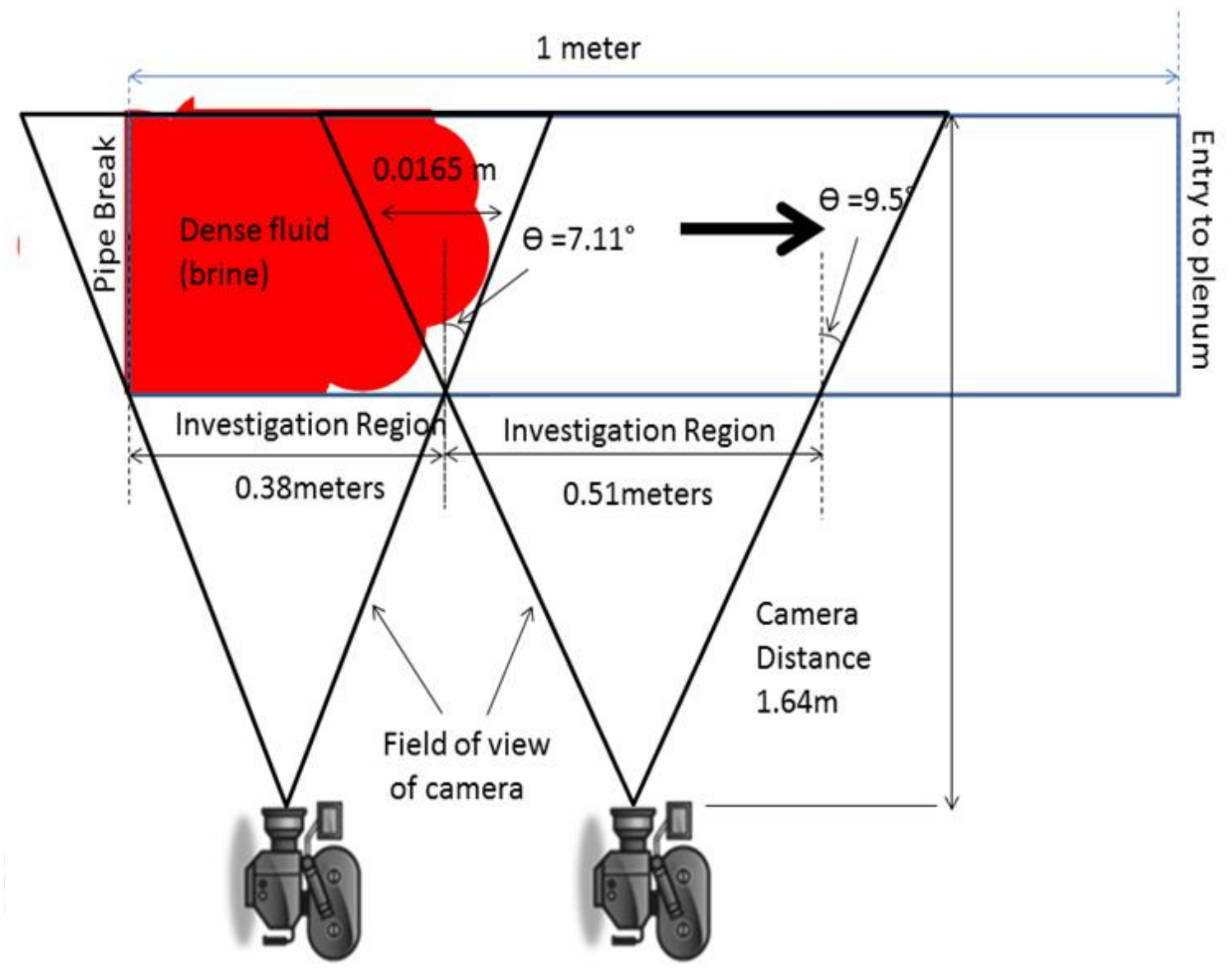

Figure 12: Uncertainty Associated with the Field of View in Case B 


\subsection{PIV Seeding Tracer Particle Uncertainty}

On the PIV measurements, a source of error is induced by gravitational forces if tracer particles' density differs largely from that of the fluid. An indication whether gravitational forces becomes important can be obtained from Stokes drag law [17], from which the gravitational induced velocity $\mathrm{U}_{\mathrm{g}}$ is given by

$$
\mathrm{U}_{\mathrm{g}}=\mathrm{d}_{\mathrm{p}}^{2} \frac{\left(\rho_{\mathrm{p}}-\rho\right)}{18 \mu} \mathrm{g}
$$

where $d_{p}$ and $\rho_{p}$ are diameter and density of the particles, $\rho$ and $\mu$ are the density and dynamic viscosity of the fluid, and $\mathrm{g}$ is the gravitational acceleration. In the PIV investigation for air ingress seen in this work, sulfur hexafluoride $\left(\mathrm{SF}_{6}\right)$ is the gas used for the working fluid and zinc stearate as the tracer particle used to monitor its flow through the tube. The large density difference is the main drive for this initial calculation. In this investigation the fluid density gradient induced a maximum gravitational velocity of $\mathrm{U}_{\mathrm{g}}=50 \mu \mathrm{m} / \mathrm{s}$ in comparison to the initial calculation using the Benjamin equation of $1.29 \mathrm{~m} / \mathrm{s}$ flow speed of the $\mathrm{SF}_{6}$ gas. Therefore the influence of fluid density changes on velocity estimation can be neglected.

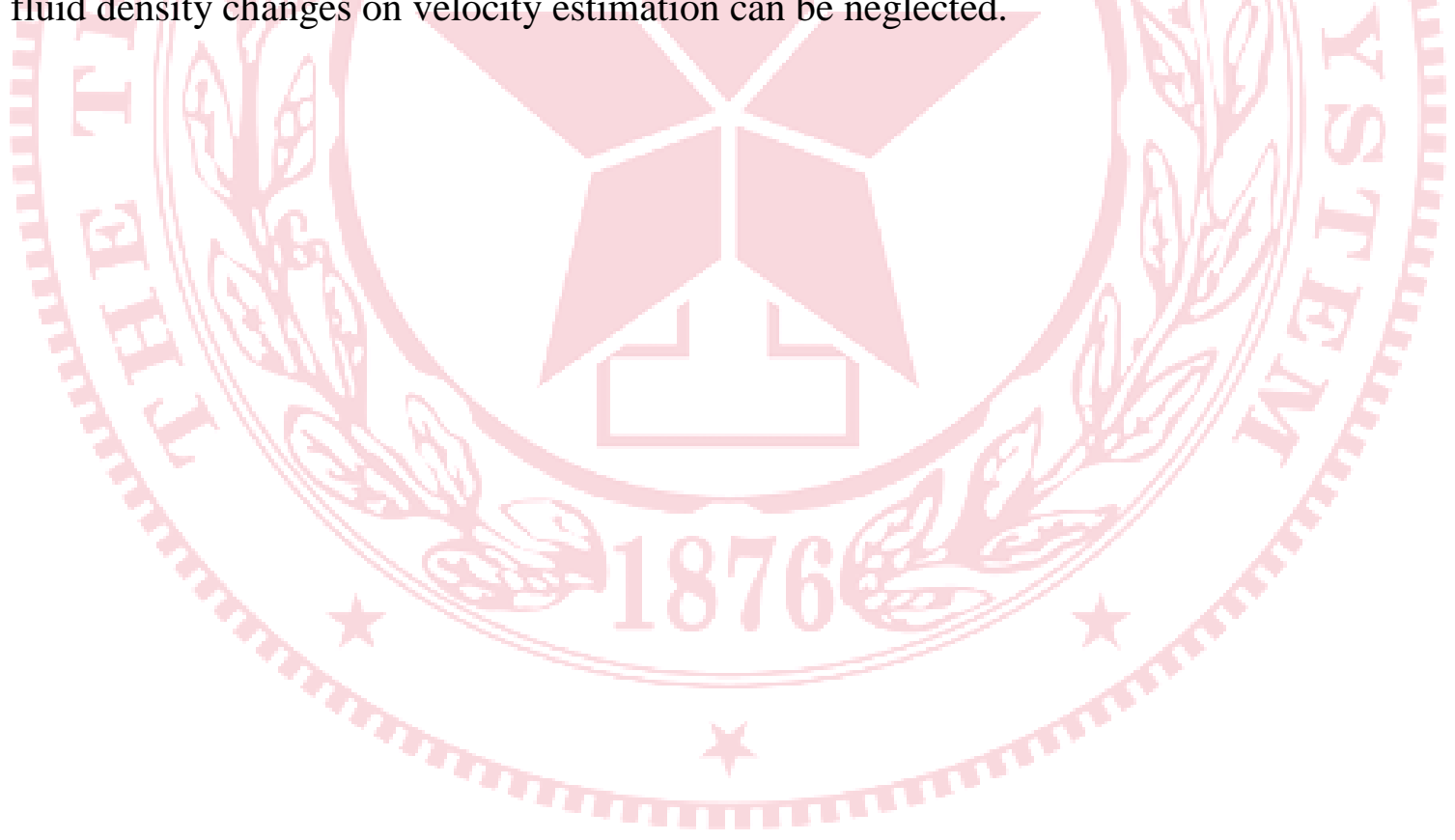




\section{CHAPTER VII}

\section{RESULTS AND DISCUSSION}

The flowing section describes separate effect experiments for understanding stratified flow phenomena in the air ingress accident scenario and the data generated for validation of CFD codes. The experiment investigates density driven stratified flow in an air ingress scenario following a double ended guillotine break (DEGB) of the large pipe connecting the reactor and the steam generator.

Three investigations were performed to develop an understanding of this stratified flow phenomenon: two liquid-liquid investigations using shadowgraphy to study the effects of varying pipe break locations and one gas-gas investigation utilizing PIV. The three experiments investigate the flow front as it propagates though the pipe after the break. In addition, the lower plenum spreading rate is investigated in the liquidliquid scenario.

\subsection{Liquid-liquid Air Ingress Investigations}

Liquid-liquid investigations are important to help understand important flow features dominant during an air ingress scenario in the scaled down experimental facility used in this investigation. The liquids used for this investigation were brine, used as the heavy working fluid (representing air ingress from reactor containment), and water as the light working fluid (representing the loss of hot coolant from the reactor core). Two investigations were conducted to investigate the effect of pipe break location. The pipe break locations are characterized based on the length of coaxial pipe that connects the simulated reactor tank to the valve. Table 3 provides a description of the pipe length cases investigated. Camera 1 captured the pipe region extending to $0.51 \mathrm{~m}$ and was adequate by itself for Case A. Because of the increase pipe length, case B required the use of two cameras to capture the flow front propagation through the pipe. Camera 1 investigation region of $0.51 \mathrm{~m}$ plus Camera 2 investigation region of $0.38 \mathrm{~m}$ with a total investigation region of $0.89 \mathrm{~m}$.

\section{Table 3: Description of Brine-Water Investigations}

\begin{tabular}{|l|l|l|l|}
\hline & DensityRatio $\left(\rho_{\text {light }} / \rho_{\text {heavy }}\right)$ & Pipe Length $(\mathrm{m})$ & Investigation Region $(\mathrm{m})$ \\
\hline Case A & 0.88 & 0.38 & 0.16 \\
\hline Case B & 0.88 & 1.0 & 0.89 \\
\hline
\end{tabular}




\subsubsection{Flow Front Analysis}

From the initial flow visualizations, the flow front was captured in the pipe and entering the lower plenum. Figures 13 and 14 shows the heavy fluid current propogating through the pipe after the valve is opened in both cases. As can be seen the density driven stratified flow model introduced in Fig. 4 appears to approximate fairly well the experimental results in this investigation. From this, the flow front speed in the coaxial pipe and spreading rate into the lower plenum was calculated. These visualizations provide insight to the short time scale it takes for a heavy fluid to move from the initiated pipe break through the pipe to the reactor and potentially cause an earlier onset of oxidation (on the order of a tens of seconds).
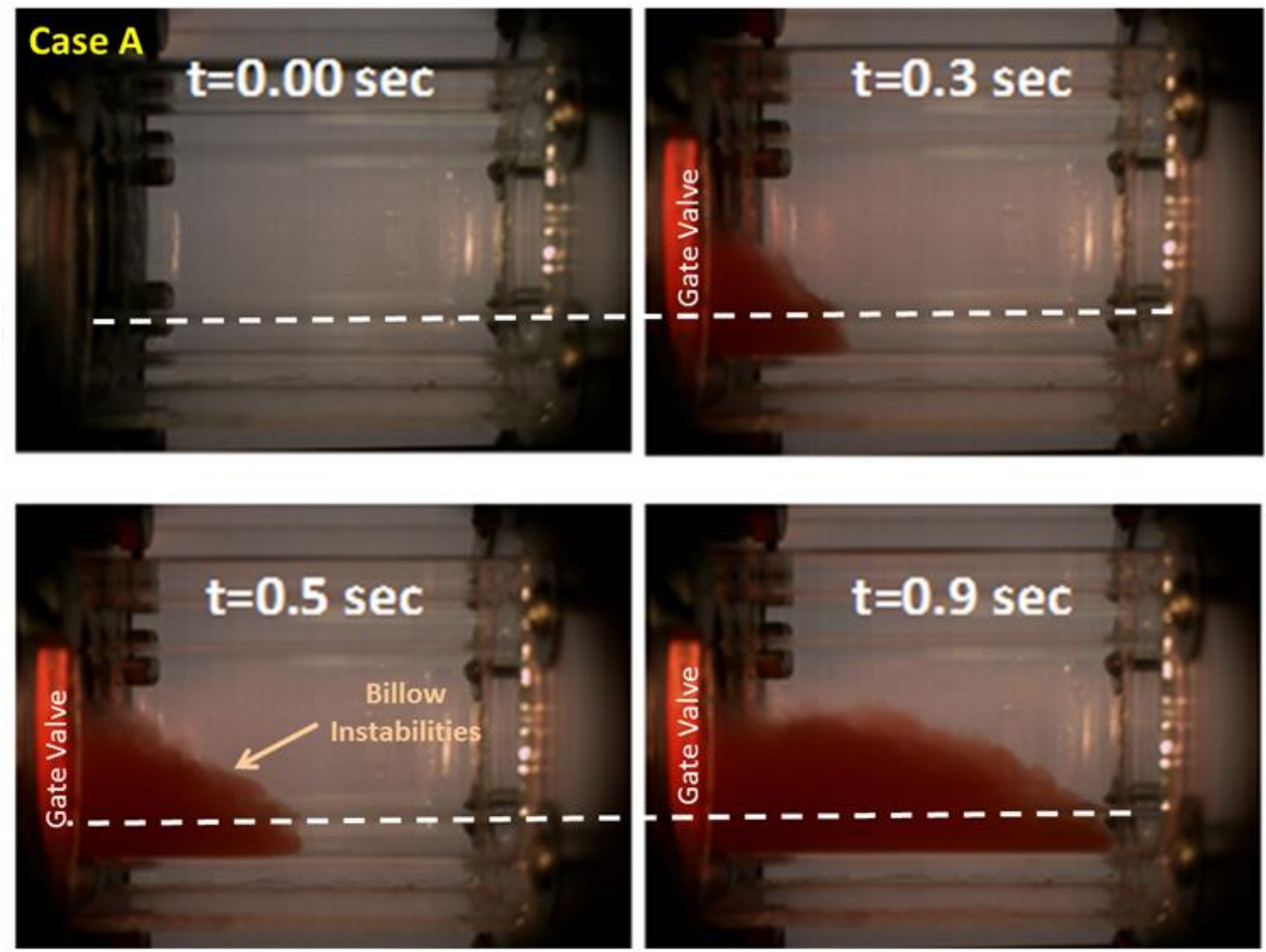

Figure 13: Progression of Gravity Currents and Stratified Flow in Case A. The Dashed Line Signifies the Inner Pipe Location 


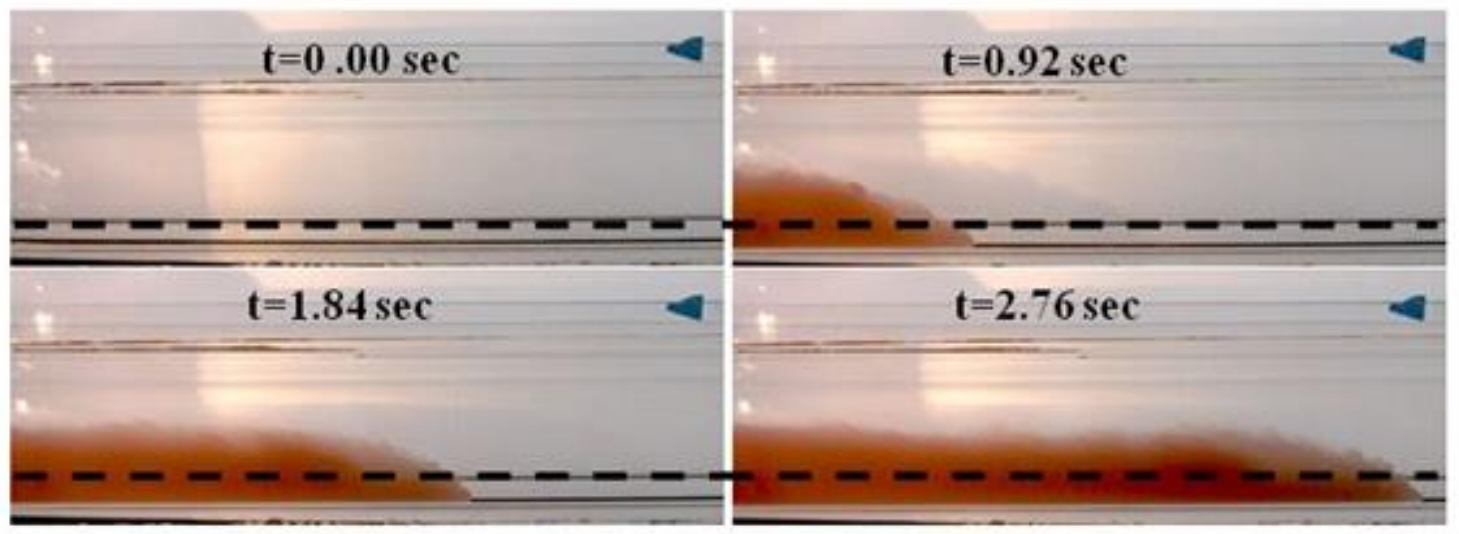

Figure 14: Progression of Gravity Currents and Stratified Flow in Case B. The Dashed Line Signifies the Inner Pipe Location

In both cases the density ratio was 0.88 , which means that the brine solution is about $13 \%$ heavier than the water which is approximately, in scaled terms, the same difference as air entering from the reactor containment area into the helium coolant would be. As shown in Figs. 13 and 14, the wave front rapidly propagates through the coaxial pipe, occupying about one-half of the pipe diameter (H in Fig. 4). Both cases have turbulence clearly present. This is expected with Case A with a Reynolds number range of 0-19563 and in case B with a Reynolds number ranges of 0-18330. Also, these visualizations show formations of billows (noted in Fig.13). As previously discussed billows are type of instabilitiy that causes mixing and with further investigaion may be classified as Kelvin-Helmholtz billows due to definition given prior.

Figure 15 shows the initial rapid burst that Simpson discussed after the gate is removed indicated from the velocity jumping from 0 to $0.24 \mathrm{~m} / \mathrm{s}$ and rapidly decelerating to constant velocity after the initial 0.07 meters of travel. At this point, phase one is initiated as seen with the nearly constant velocity as the fluid propagates in the axial direction. Figure 16 shows the same initial burst for Case B. 


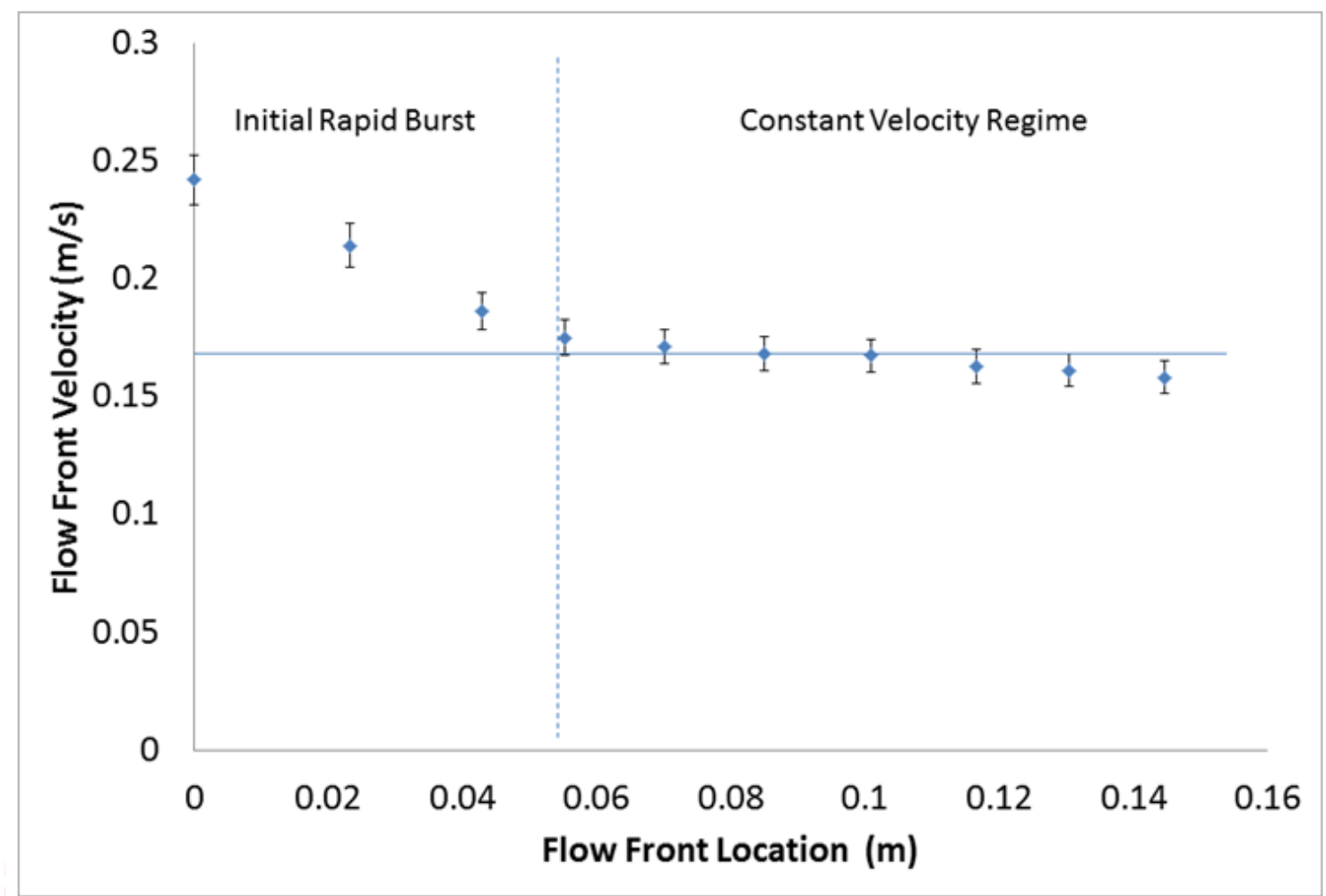

Figure 15: Experimental Results for the Pipe Flow Front Velocity versus Location in Case A 


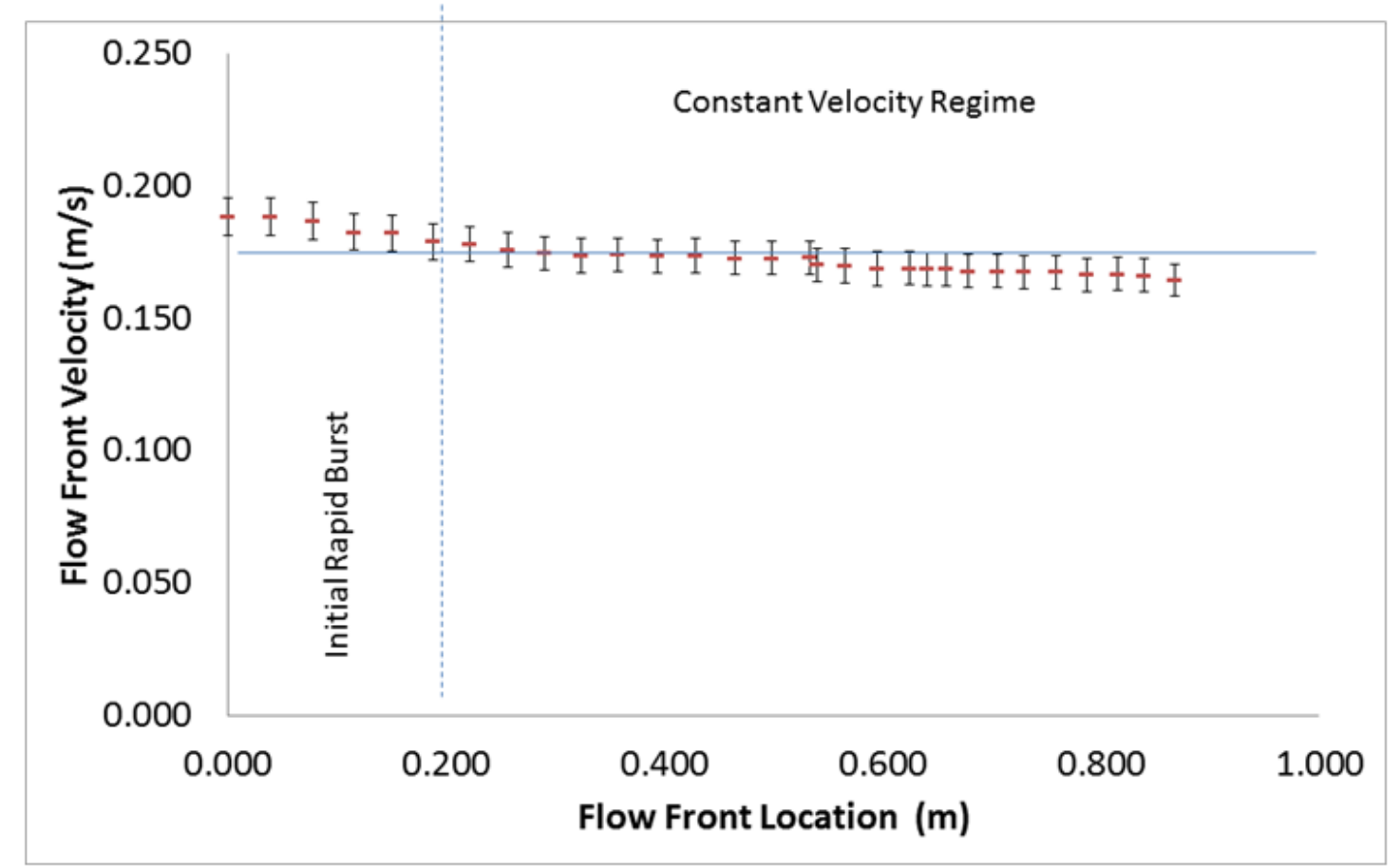

Figure 16: Experimental Results for the Pipe Flow Front Velocity versus Location in Case B

The heavy flow front velocity is consistent with the previous observations reported for the lock exchange flow in the Boussinesq flow regimes following Benjamin's equation [9], Eq. 2. In both previous evaluations, the theoretical heavy flow front velocity was estimated to be $\sim 0.1608 \mathrm{~m} / \mathrm{s}$ from Benjamin's equation. The velocities are the same for both previous evaluations due to the dependence of Benjamin's equation on the diameter only of the pipe and not on the break location.

Table 4 shows the comparison of the flow front velocities between the current experimental values and Benjamin's theoretical value. In both Case A and Case B, the experimental front velocities are in good agreement with Benjamin's theoretical calculation (within estimated measurement uncertainty). 
Table 4: Comparison of Flow Front Velocity between Experimental and Benjamin's Theoretical Model

\begin{tabular}{|lcl|}
\hline \multicolumn{3}{|c|}{ Flow Front Velocity $(\mathrm{m} / \mathrm{s})$} \\
\hline & Case A & Case B \\
Experimental & $0.164 \pm 4.29 \%$ & $0.168 \pm 3.69 \%$ \\
Benjamin's Theory & 0.1608 & 0.1608 \\
Error $(\%)$ & 1.99 & 4.5 \\
\hline
\end{tabular}

Another part of this experiment was to gain confidence in the CFD calculations by comparison with the experimental values obtained. Table 5 shows the comparison of the flow front velocities between experimental and CFD results. At this time only CFD results for case A have been completed. The flow front velocity difference is low when comparing experimental and CFD results. Therefore, it presents that the CFD model is adequately simulating the phenomenon in this lock exchange density driven stratified flow.

Table 5: Comparison of Flow Front Velocity between Experimental and CFD

\begin{tabular}{|lll|}
\hline \multicolumn{3}{|c|}{ Flow Front Velocity $(\mathrm{m} / \mathrm{s})$} \\
\hline & Case A & Case B \\
Experimental & $0.164 \pm 4.29 \%$ & $0.168 \pm 3.69 \%$ \\
CFD & 0.168 & 0.172 \\
Difference (\%) & 2.4 & 2.4 \\
\hline
\end{tabular}

Flow front velocity and spreading rate measurements were conducted using high speed cameras and image processing software. Further analysis was done to measure change in position of the flow front relative to time and it's velocity as a function of position. Case A used one camera for the pipe location and one to investigate the lower plenum (125 frames per second). Case B used two cameras for the pipe location (250 fps and $300 \mathrm{fps}$ ) and one to investigate the lower plenum (150 fps). Flow front velocity measurements were conducted with the image processing software by using the simple equation seen in Eq. (9):

$$
V=\frac{d x}{d t}
$$


Distance of the investigation area was measured prior to the experiment and the frame rate is known. Using the image processing software pixels were correlated to distance covered in a time stamp. This time stamp was calculated by the frame rate of each camera. The results are seen in Figure 17.

According to Fig. 17, after the initial burst, the flow front location progresses linearly with time for both cases. This linear relation of flow front location with time indicates a constant heavy flow front speed axially through the coaxial pipe. This phenomenon was also observed and documented by Benjamin in his investigation of heavy salt water solution displacing the lighter fluid, water [9]. Simpson [14] shows this constant velocity is also believed to be the first phase of a lock exchange flow after the initial gate removal. Figure 17 shows a constant flow front velocity indicating steady flow conditions.

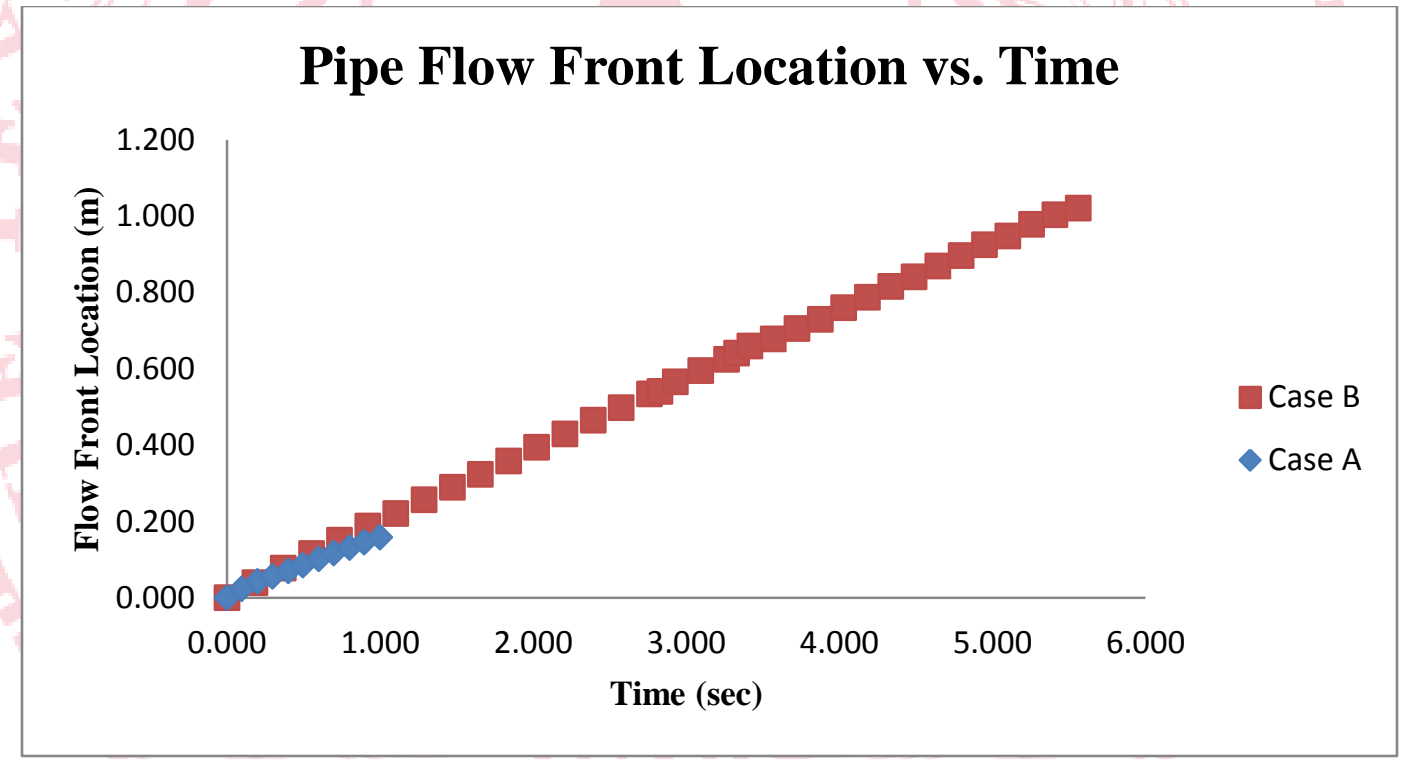

Figure 17: Experimental Results for the Pipe Flow Front Location versus Time

As it exits the pipe, the flow moves into the lower plenum of the reactor cavity. Figure 18 shows the dense fluid (simulated air ingress into reactor core) propagating into the lower plenum from the coaxial pipe. In Fig. 18, the camera is positioned above the lower plenum and ingress through the hot duct is indicated by flow arrow. 


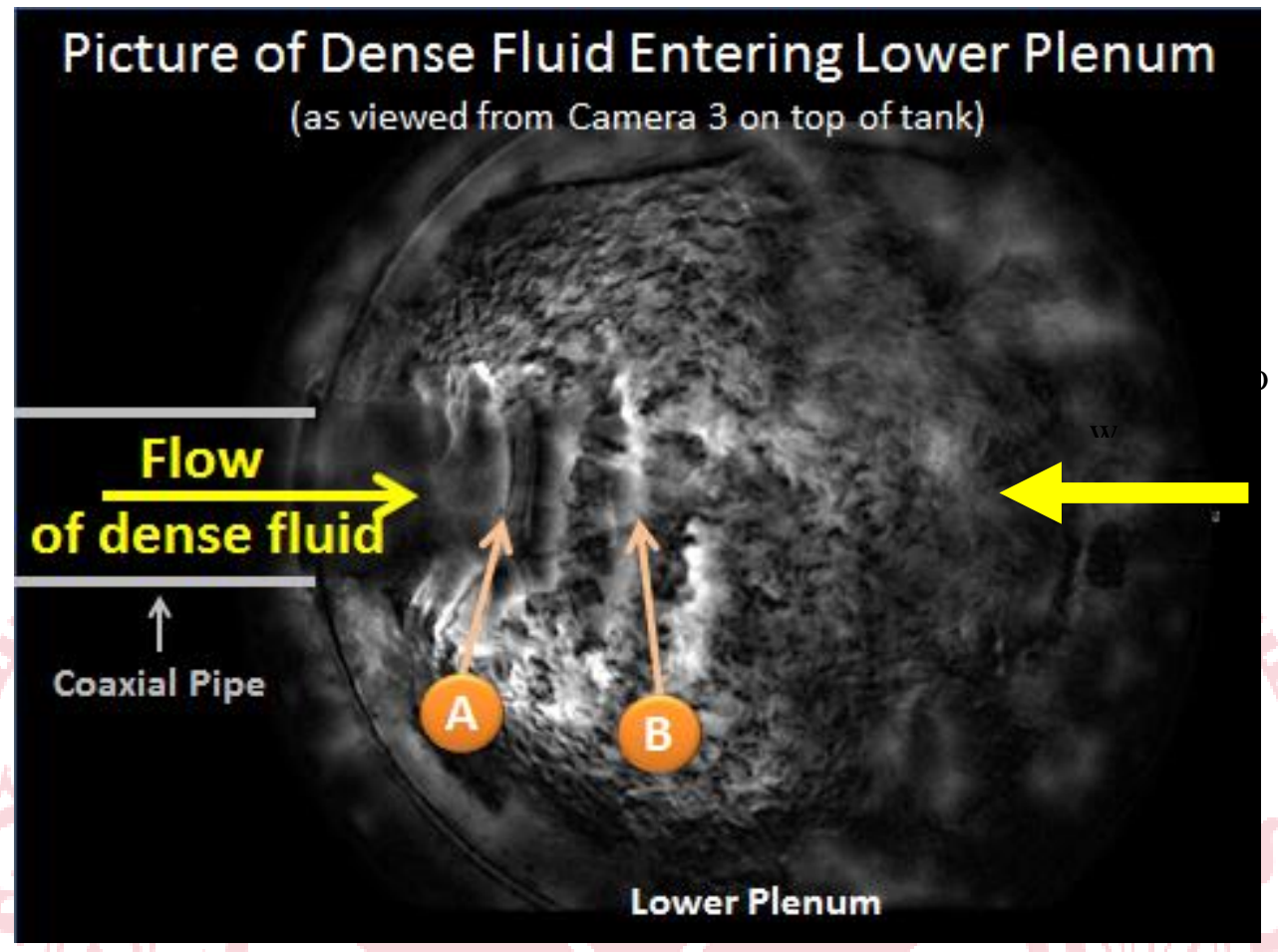

Figure 18: Highly Periodic Interfacial Instabilities between the Brine and Water

Following the initial break, the gravity driven flow spreads into the lower plenum, creating Kelvin-Helmholtz instabilities at the interface between the heavy and light flows just after the entrance region. The initial folding of waves is indicated by ${ }^{6} \mathrm{~A}$ ' in Fig. 18, which eventually leads to a breaking wave at point 'B.' In addition to the formation of a breaking wave pattern, rib vortices are formed transverse to the waves and move outward towards the edges of the wave as it propagates.

From this wave propagation, similar calculations were conducted for flow into the lower plenum of both cases. These are seen in Figs. 19 and 20. Figure 18 shows how the fluid enters the lower plenum and spreads at a constant velocity. This is the outcome of both Case A and B.

The same shape can also be seen in the lower plenum as in the pipe as seen in Fig. 20. The flow goes from a smaller area into a larger area creating an initial burst of speed and then the spreading rate becomes relatively constant as it moves through the tank. 


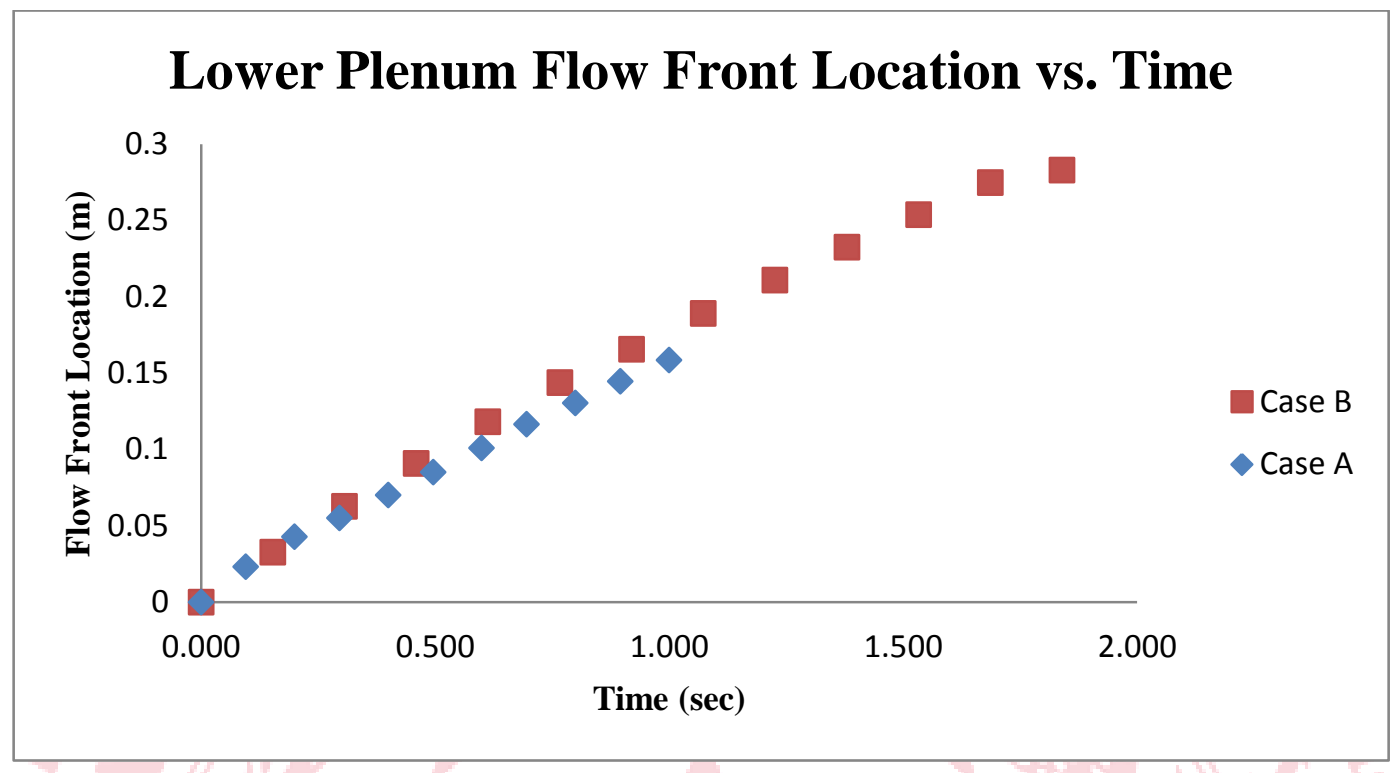

Figure 19: Experimental Results for Flow Front Location versus Time of the Lower Plenum

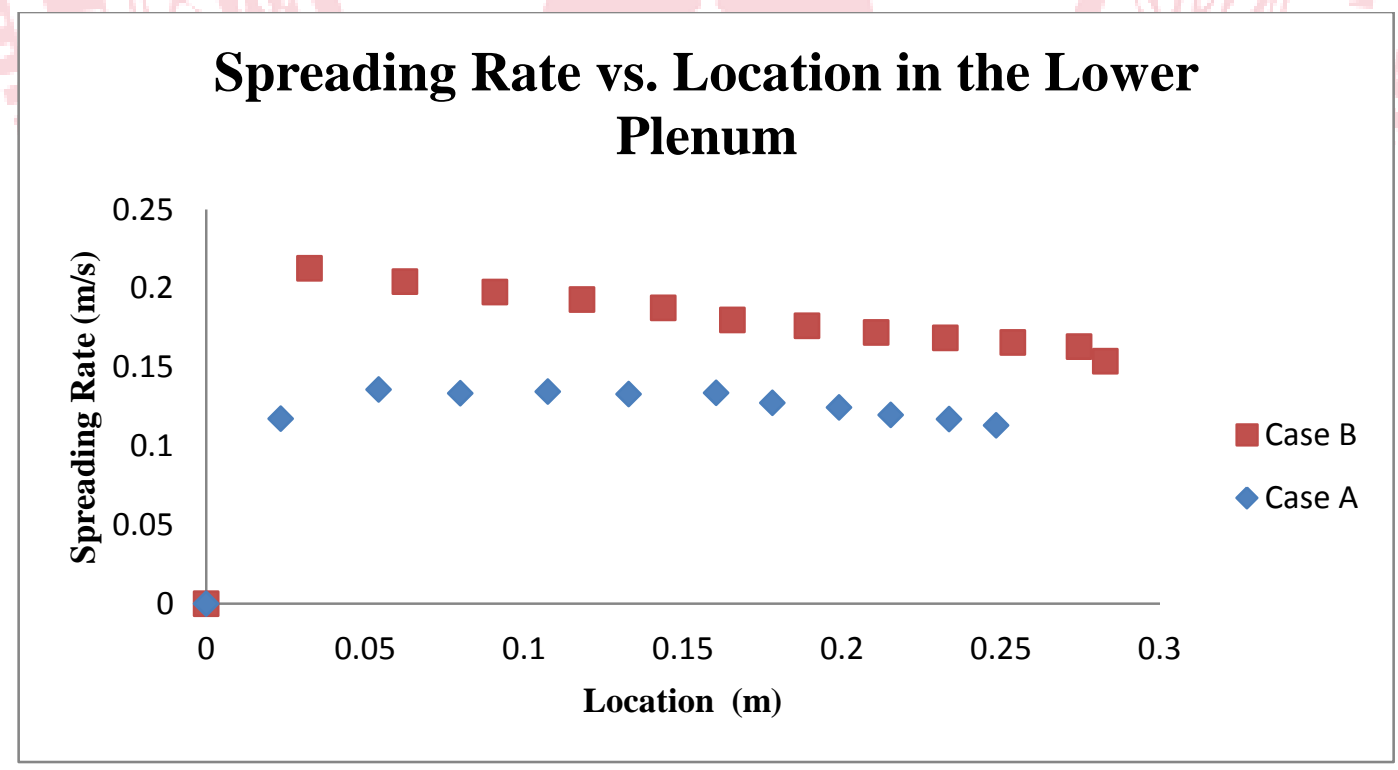

Figure 20: Experimental Results for Spreading Rate versus Location in the Lower Plenum 
The time it takes for the flow to reach the lower plenum is a very short time period for both investigations. For case A the heavy brine solution reaches the lower plenum in 3.04 seconds and for case B it takes 5.2 seconds. This short timescale reveals the short mitigation time that is avalable if this event were to occur.

\subsection{Gas-gas Air Ingress Investigation}

PIV investigations have been initiated to analyze the flow front of the gas-gas scenarios. Currently the first PIV tests have been conducted with $\mathrm{SF}_{6}$ as the heavy working fluid and helium as the light. Zinc stearate particles are used as the chosen seeding particles to follow the $\mathrm{SF}_{6}$ gas due to their relatively light density and particle distribution which can be seen in the next section.

In order to compare the velocity and time scale of density driven stratified flow from PIV data, diffusion time scale and diffusion velocity was calculated. Diffusion time scale and velocity was calculated for both gas scenarios, $\mathrm{SF}_{6} / \mathrm{He}$ and $\mathrm{CO}_{2} / \mathrm{He}$. The $\mathrm{SF}_{6} / \mathrm{He}$ gas case is the only comparison available at this time since the $\mathrm{CO}_{2} / \mathrm{He}$ gas case has not been run at this time. Equation 10 is the equation used to calculate the binary diffusion coefficient [18]. Equation 11 and 12 gives the diffusion time scale and diffusion velocity respectively.

$$
\begin{aligned}
& D_{\text {SF6-He }}=\frac{2.628 \times 10^{-7} T^{3 / 2}}{\left(\mathrm{P \sigma}_{\mathrm{SF} 6-\mathrm{He}}^{2} \Omega_{\mathrm{D}} \mathrm{M}_{\mathrm{SF} 6-\mathrm{He}}^{1 / 2}\right)} \\
& \mathrm{t}_{\text {Diff }}=\frac{\mathrm{L}^{2}}{\mathrm{D}_{\mathrm{SF} 6-\mathrm{He}}} \\
& \mathrm{V}_{\text {Diff }}=\frac{\mathrm{L}}{\mathrm{t}_{\text {Diff }}}
\end{aligned}
$$

The diffusion time and diffusion velocity are compared to the experimental values of the density-driven stratified flow front. These values can be seen in Table 6 . The heavy flow front of the density-driven stratified flow was measured from the PIV data. This velocity is two orders of magnitude larger than the diffusion velocity. This shows the differing importance of the two mechanisms and the reason density-driven stratified flow needs to be investigated. 
Table 6: Comparison of Diffusion Velocity to Density-driven Stratified Flow

\begin{tabular}{|lll|}
\hline & $\mathrm{SF6}-\mathrm{He}$ & $\mathrm{CO} 2-\mathrm{He}$ \\
\hline $\mathrm{t}_{\text {Diff }}(\mathrm{s})$ & 195.4 & 141.1 \\
$\mathrm{~V}_{\text {Diff }}(\mathrm{m} / \mathrm{s})$ & 0.0046 & 0.0063 \\
$\mathrm{u}_{\text {Heavy }}(\mathrm{m} / \mathrm{s})$ & 0.31 & ---- \\
\hline
\end{tabular}

\subsubsection{PIV Seeding Analysis}

An important factor in PIV is the ability of the seeding particles, or tracer particles, to move with the flow. Thus, the selection of particles is key to ensure an accurate representation of the flow features present in the air ingress scenario. Zinc stearate seeding particles were chosen based on particle size distribution and density. The average particle size is $\sim 2 \mu \mathrm{m}$ with a density of $400 \mathrm{~kg} / \mathrm{m}^{3}$.

It is important to ensure that particles are the particular size obtained from the manufacturers. Normally a manufacturer gives the particle diameter according to the largest particle diameter but not the distribution. It is important to know the distribution of the particles to ensure the correct particles are chosen.

Particle size distributions of various manufacture samples were performed using a TSI Aerodynamic Particle Sizer (APS model number 3321) to ensure the particles adhered to the manufacturer specifications. The particle size distributions of two manufacturers, Ferro and Struktol, are presented in Figs. 21 and 22 respectively. It can be seen that the zinc stearate particles from the Ferro manufacturers has a more consistent distribution with a peak approximately at 1.7 micrometers. Figure 22 shows a less consistent distribution with bi-modal distribution with peaks present at 0.8 and 1.7 micrometers. From this particle analysis of these two particles, Ferro, the more consistent particle distribution is chosen as the particles to use in the investigation. 


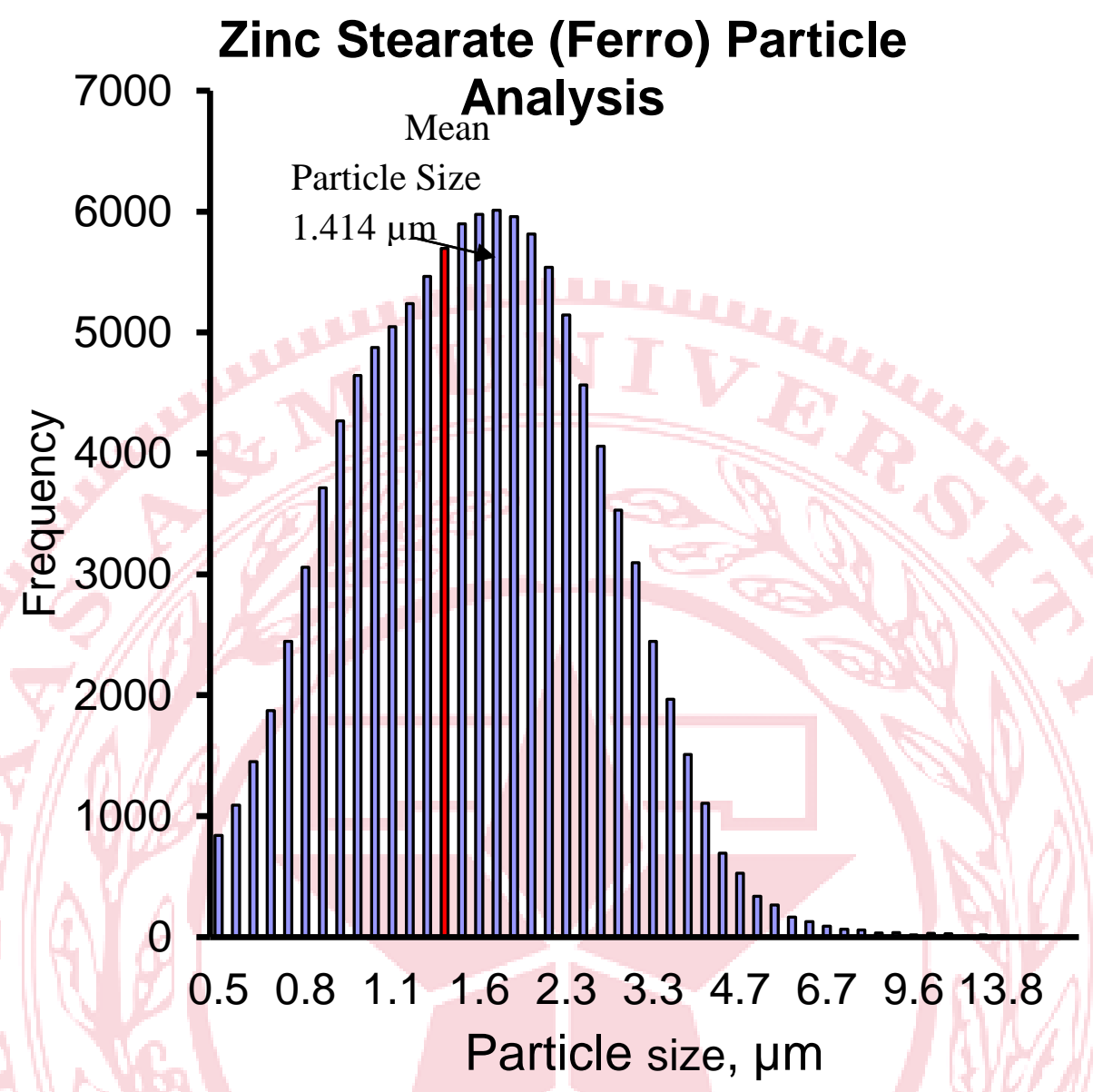

Figure 21: Zinc Stearate Particle Analysis (Ferro) 


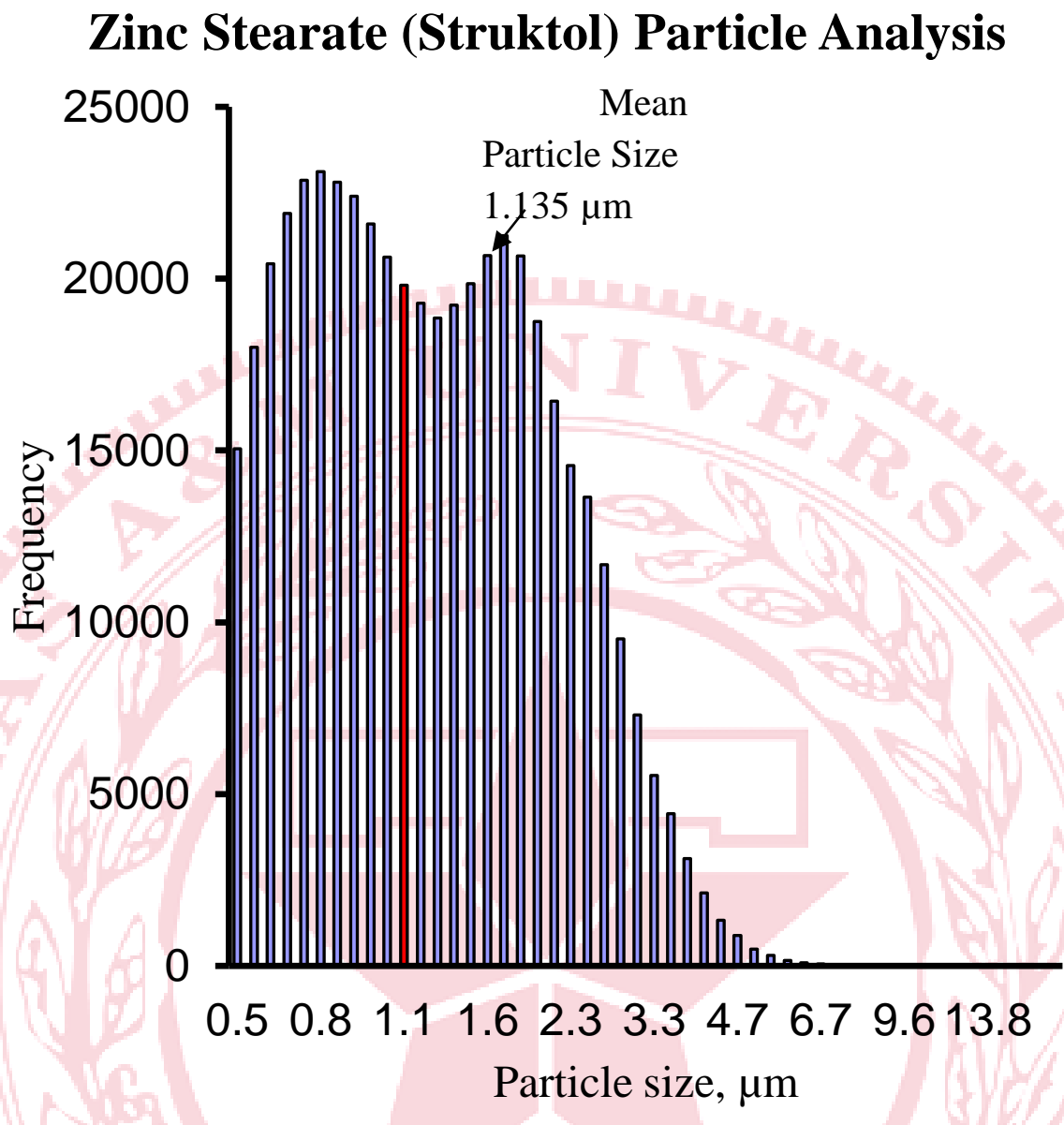

Figure 22: Zinc Stearate Particle Analysis (Struktol)

\subsubsection{Experimental Imaging Analysis}

Currently it is being performed the methodology required for a complete and accurate analysis of the PIV images. Among the major challenges to be tackled is the need of a huge PIV dynamic velocity range to measure correctly velocities at the beginning of the transient. The PIV dynamic range corresponds to the ratio of the maximum to the minimum resolvable velocity by the PIV algorithm. The helium-sf6 experiments showed to have a dynamic velocity range of about 160 . This value was obtained assuming that the minimum resolvable velocity is on the order of 0.5 pixels. However, with the commonly used sub-pixel interpolation, it is claimed that displacements of about 0.1 pixels or lower can be obtained. Therefore, with subpixel interpolation the dynamic range for this specific case may be as high as 800 , which is out of the scope of traditional PIV algorithms. This effect can be seen in Fig. 23 on which 
the time evolution of the horizontal component of the velocity is displayed. In the first $250 \mu \mathrm{s}$, the fluid experiences a change from stagnation to a velocity up to $8 \mathrm{~m} / \mathrm{s}$ after which an oscillatory behavior is experienced. These sudden changes in acceleration imply large particle image displacements on the PIV experimental images, of the order of 85 pix in between each frame. Traditional PIV algorithms are unable to extract accurate velocity information in such conditions. To this end, a Particle Tracking Velocimetry (PTV) algorithm is being modified to fulfill the demands of the SF6-He experiments.

As shown in Figure 24, the experiment was performed with four synchronized high speed cameras, each of which captured a different region of interest. This allowed the experimenter to study different areas of the test section with a higher spatial resolution. Examples of the instantaneous experimental images are shown from Fig. 25(a) to Fig. 28(a). Although multiple viewing sections provided a detailed description of the "air-ingress" phenomena, the different camera characteristics makes their analysis unique and difficult. The same PTV algorithm parameters cannot be used to analyze results from two different cameras. Variables that have to be considered are the different spatial resolution, different camera light sensitivity, different cameras spatial calibration, different exposure times, and even different lens errors effects. Currently, a sensitivity study is being performed to obtain image processing and image analysis parameters that will suit each individual camera result. A preliminary analysis with default PTV algorithm parameters is shown from Fig. 25(b) to Fig. 28(b). The detection of spurious vectors is to be reduced once the sensitivity study on the algorithm parameters is finished. Although the difficulty of having such a large dynamic velocity range, the results from the PTV analysis showed to be reasonable. Further analysis is required to obtain the proper procedure for the statistical analysis required to better describe the transient phenomenon with such large range of time scales. 


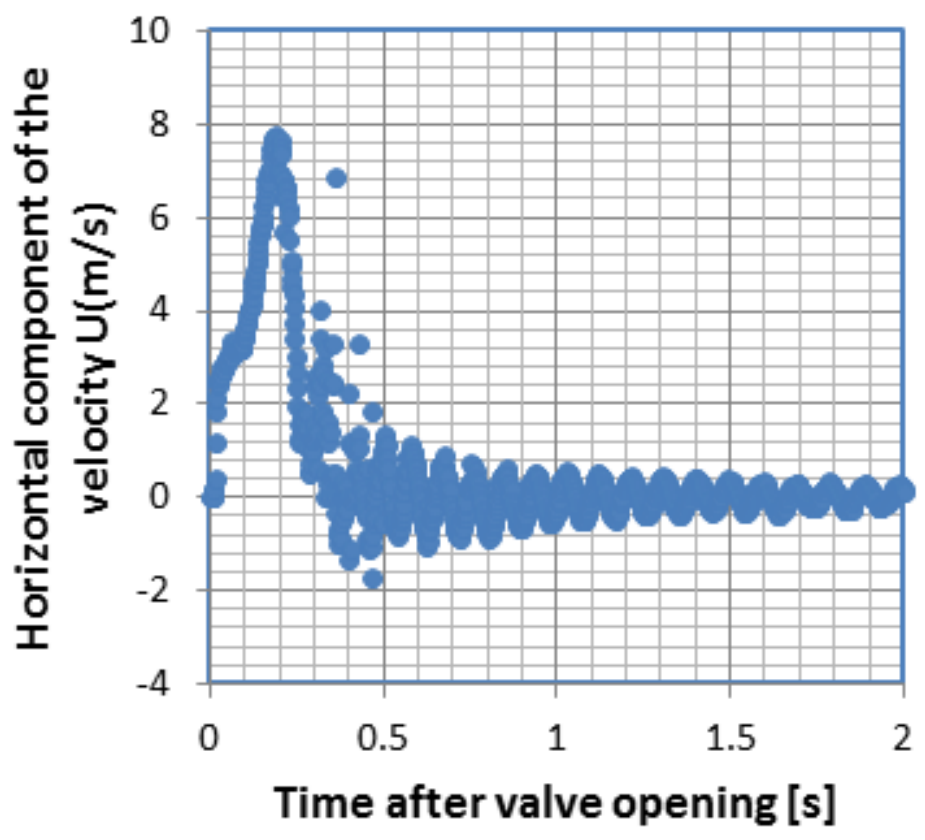

Figure 23: Time evolution of average horizontal component of the velocity (Camera 2)

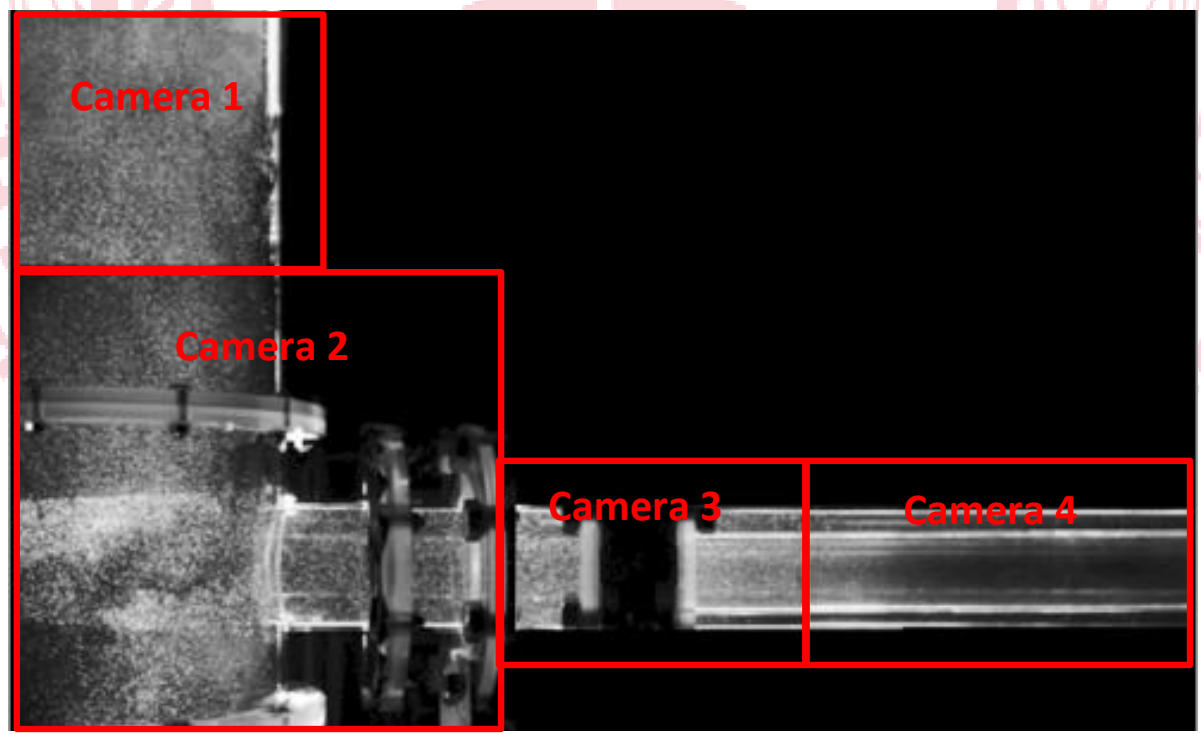

Figure 24: Overlay of the PIV experimental results from four synchronized cameras. 


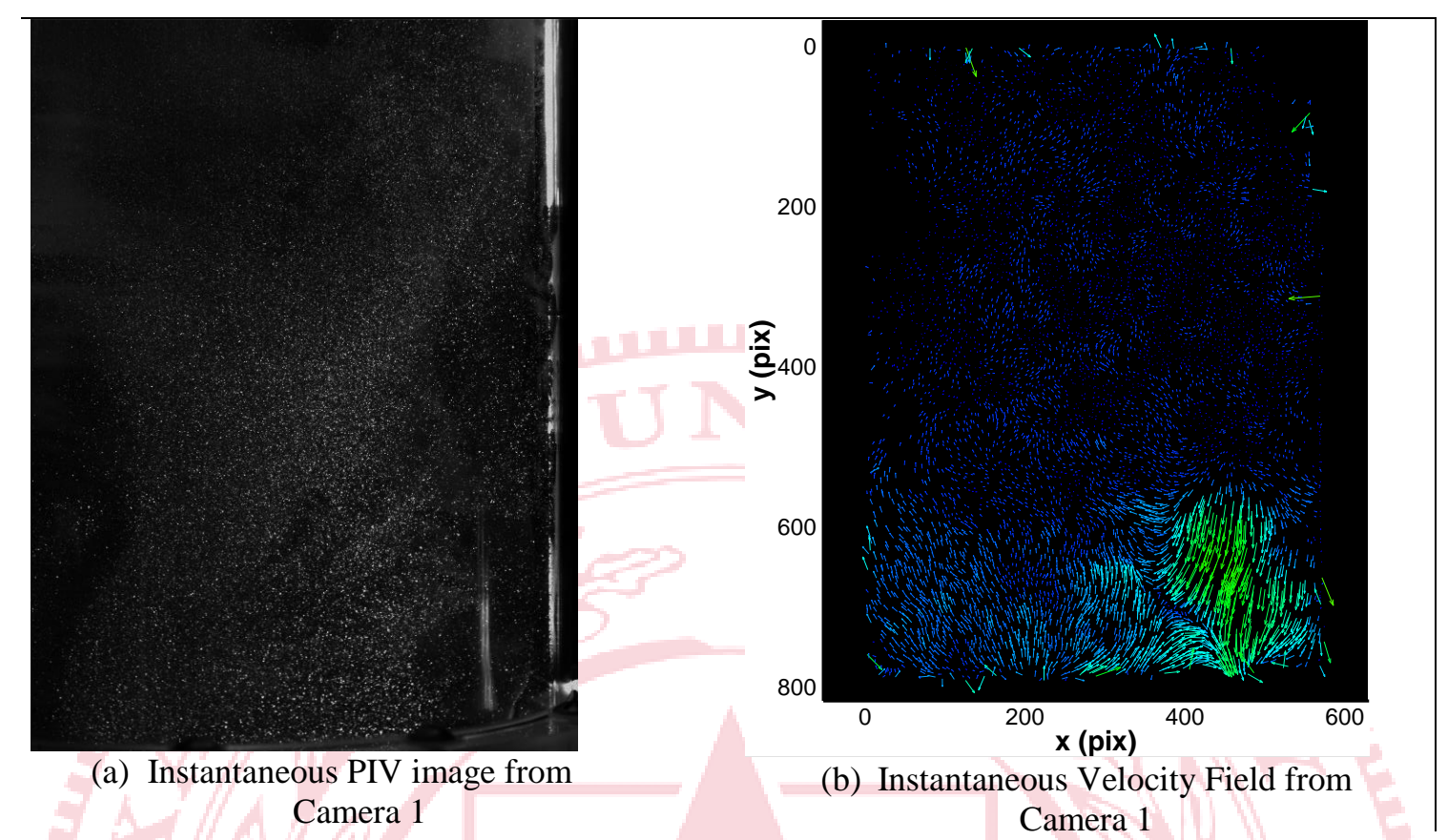

Figure 25: Experimental results and analysis of data from camera 1

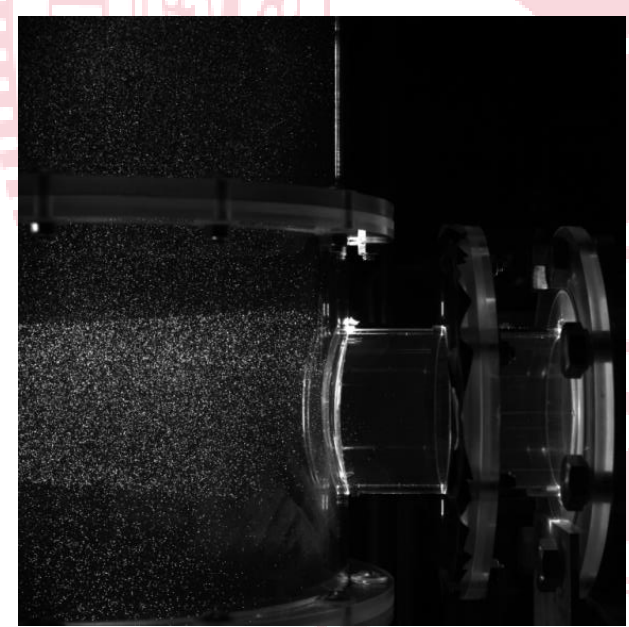

(a) Instantaneous PIV image from

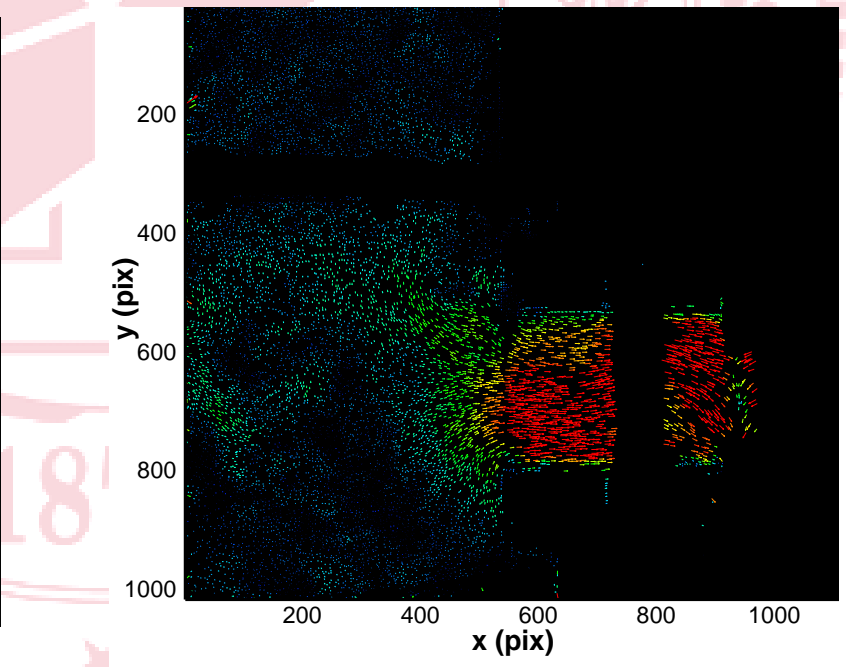

(b) Instantaneous Velocity Field from Camera 2

Figure 26: Experimental results and analysis of data from camera 2 


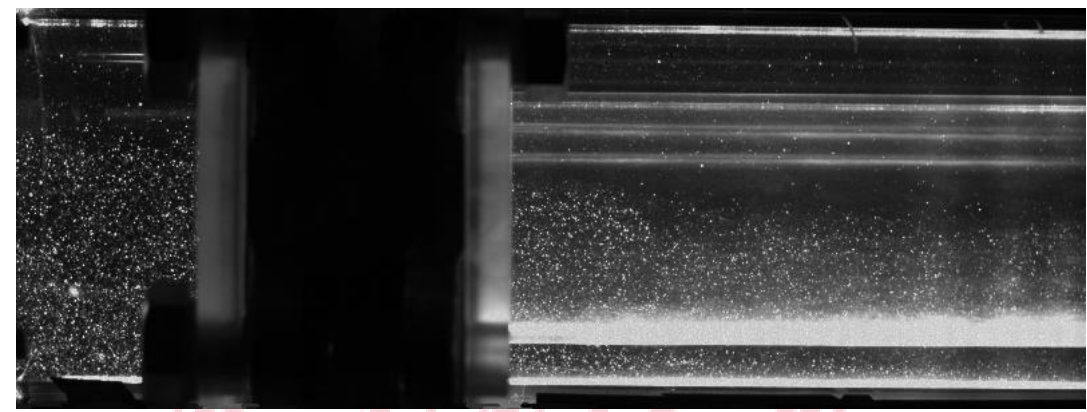

(a) Instantaneous PIV image from Camera 3

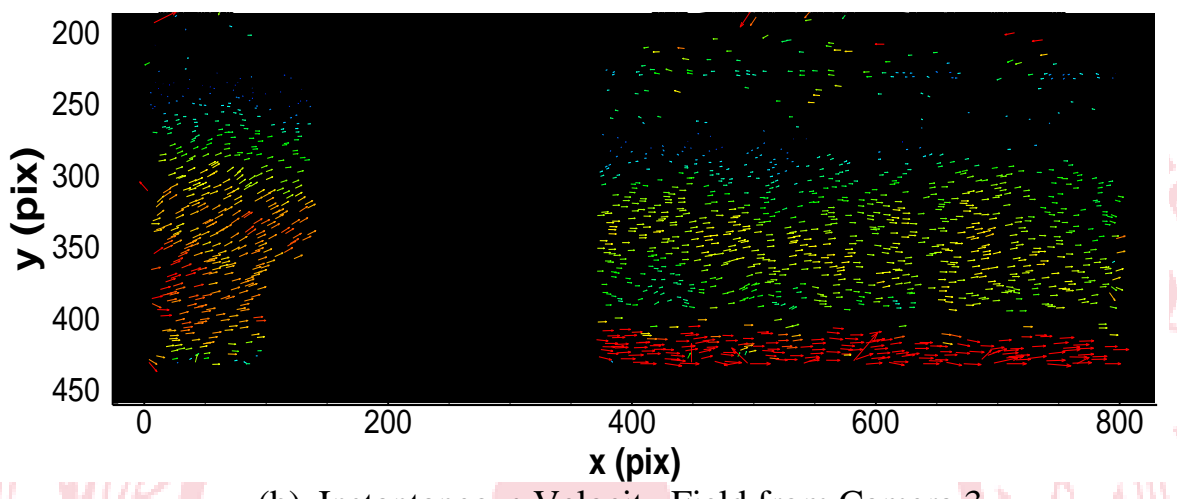

(b) Instantaneous Velocity Field from Camera 3

Figure 27: Experimental results and analysis of data from camera 3

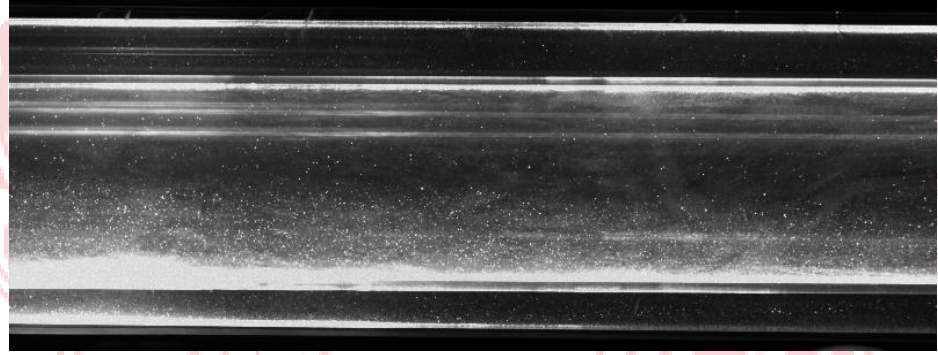

(a) Instantaneous PIV image from Camera 4

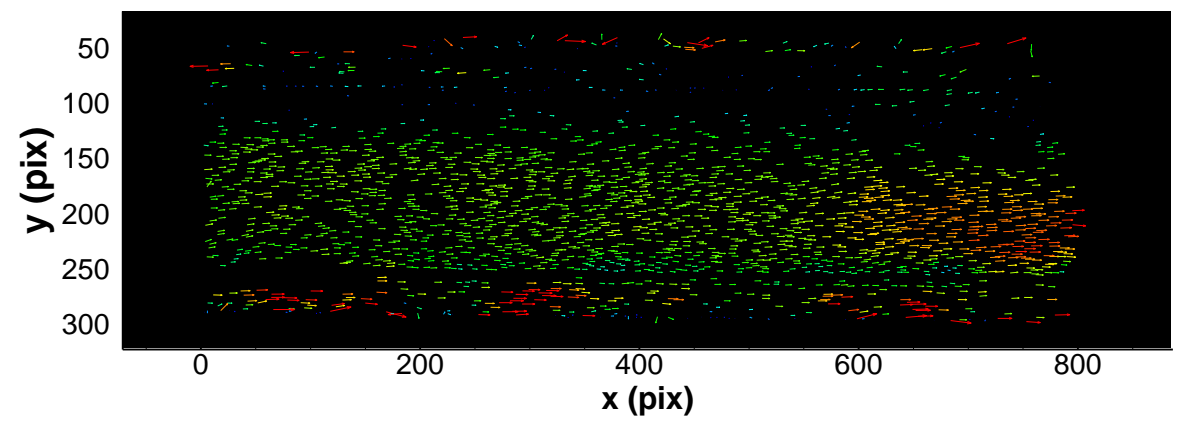

(b) Instantaneous Velocity Field from Camera 4

Figure 28: Experimental results and analysis of data from camera 4 


\section{CHAPTER VIII}

\section{SUMMARY AND CONCLUSIONS}

Due to the differing time scales of interaction, the air ingress mechanism, whether it is dominated by diffusion or density-driven stratified flow need to be verified. Air ingress could possibly result in oxidation of in-core graphite structures and fuel, potentially collapsing the bottom structures of the core and releasing fission products and hazardous levels of carbon monoxide [2]. The shorter the time scale indicated by the density driven stratified flow causes a faster onset of natural circulation leading to earlier graphite oxidation. This earlier onset of oxidation provides less time for outside mitigation.

From the experimental investigation, the gravity driven ingress mechanism is verified as being a shorter time scale by using selected fluids. Brine was used as the heavy fluid and water as the light with a density ratio of 0.88 . The experiment shows stratification of the two fluids. The flow front analysis for the coaxial pipe is in agreement with Benjamin's theoretical value and with CFD results.

The time it takes for the flow to reach the lower plenum is a very short time period for both investigations. Both cases results in the heavy fluid entering the lower plenum in under 6 seconds. This short timescale reveals the short mitigation time that is avalable if this event were to occur.

The diffusion velocity and heavy flow front of the stratified flow layer were compared for the $\mathrm{SF}_{6} / \mathrm{He}$ gas case. It is seen that the diffusion plays less of a role as the transport mechanism in comparison to the density-driven stratified flow since the velocity of the diffusion is two orders of magnitude smaller than the velocity of the stratified flow mechanism. This is the reason for the need for density-driven stratified flow investigations following a LOCA. 


\section{REFERENCES}

[1] U.S. DOE Nuclear Energy Research Advisory Committee and the Generation IV International Forum, 2002, “A Technical Roadmap for Generation IV Nuclear Energy Systems," Generation IV International Forum.

[2] Oh, C. H, Kim, E. S., NO, H. C, Cho, N. Z., 2008 "Experimental Validation of Stratified Flow Phenomena, Graphite Oxidation, and Mitigation Strategies of Air Ingress Accidents," INL/EXT-08-14840, Idaho National Laboratory, Idaho Falls, ID.

[3] Oh, C., Davis, C., Siefken, L., Moore, R., NO, H., Kim, J., Park, G.C., Lee, J., and Martin, W., 2006, "Development of Safety Analysis Codes and Experimental Validation for a Very High Temperature Gas-Cooled Reactor," Final Report, INL/EXT-06-01362, Idaho National Laboratory, Idaho Falls, ID.

[4] Oh, C., Kim, E., Schultz R., Petti D., 2009, “Computational Fluid Dynamics Analyses on Very High Temperature Reactor Air Ingress," ICONE 17-75863 $17^{\text {th }}$ International Conference on Nuclear Engineering, July 12-16, Brussels, Belgium.

[5] Oh, C. H, Kim, E. S., Schultz, R., Petti, D., and Liou, C. P., 2008, "Implications of Air Ingress Induced by Density-Difference Driven Stratified Flow," 8023, International Congress on Advances in Nuclear Power Plants, pp. 313-322.

[6] Schultz, R., Nigg, D., Johnson, R., Oh, C., Johnsen, G., 2006, "Next Generation Nuclear Plant Methods Technical Program Plan,” INL/EXT-06-11804, Rev 0.26, Idaho National Laboratory, Idaho Falls, ID.

[7] Williams, B., Liou, C., Schultz, R., Kadakia, H., Phoenix, B., Horrocks, D., 2007, "Providing the Basis for Innovative Improvements in Advanced LWR Reactor Passive Safety Systems Design: An Educational R\&D Project," Idaho State Univ. Pocatello, ID, Final Report-DE-FG07-03ID14500.

[8] Oh, C. H and Kim, E. S, 2010, "Isothermal Air Ingress Validation Experiments at Idaho National Laboratory: Description and Summary of Data," INL/EXT-1019727, Idaho National Laboratory, Idaho Falls, ID.

[9] Benjamin, T. B., 1968, “Gravity Currents and Related Phenomena,” J. Fluid Mechanics, 31, pp. 209-248.

[10] Duncan, J. B., and H. L. Toor, 1962, "An Experimental Study of Three Component Gas Diffusion," A.I.Ch.E. Journal, 8, pp. 1-38.

[11] Lakehal, D., Lemonnier, H., 2003, “Test-case Number 28: The Lock-Exchange Flow,” Nuclear Engineering Laboratory, Zurich, Switzerland, March 17, 2003. 
[12] Shin, J.O., Dalziel, S. B., Linden, P.F., 2004, "Gravity Currents Produced by Lock Exchange,” J. Fluid Mech. 521, pp. 1-34.

[13] Simpson, J. S., 1997, "Chapter 11: The Anatomy of a Gravity Current and Chapter 12 Spread of Dense Fluid," Gravity Currents in the Environment and the Laboratory, 2nd Edn. Cambridge University Press, Cambridge, UK.

[14] Turner, J.S., 1973, Buoyancy Effects in Fluids, Cambridge Monographs on Mechanics and Applied Mathematics. Cambridge University Press, Cambridge, UK, pp. 70-74.

[15] Parsons, J., "Conservative Gravity Currents," Ocean Engineering, University of Washington, Seattle, WA.

[16] Adrian, R., 1991, "Particle-Imaging Techniques for Experimental Fluid Mechanics," Annu. Rev. Fluid Mech., 23, pp. 261-304.

[17] Riethmuller, M.L., 2000, "Particle Image Velocimetry and Associated Techniques," von Karman Institute for Fluid Dynamics, Genese, Belgium.

[18] Annamalai, K. and Puri, I.K., 2007, "Chapter 6: Mass Transfer," Combustion Science and Engineering, CRC Press, Boca Raton, FL.

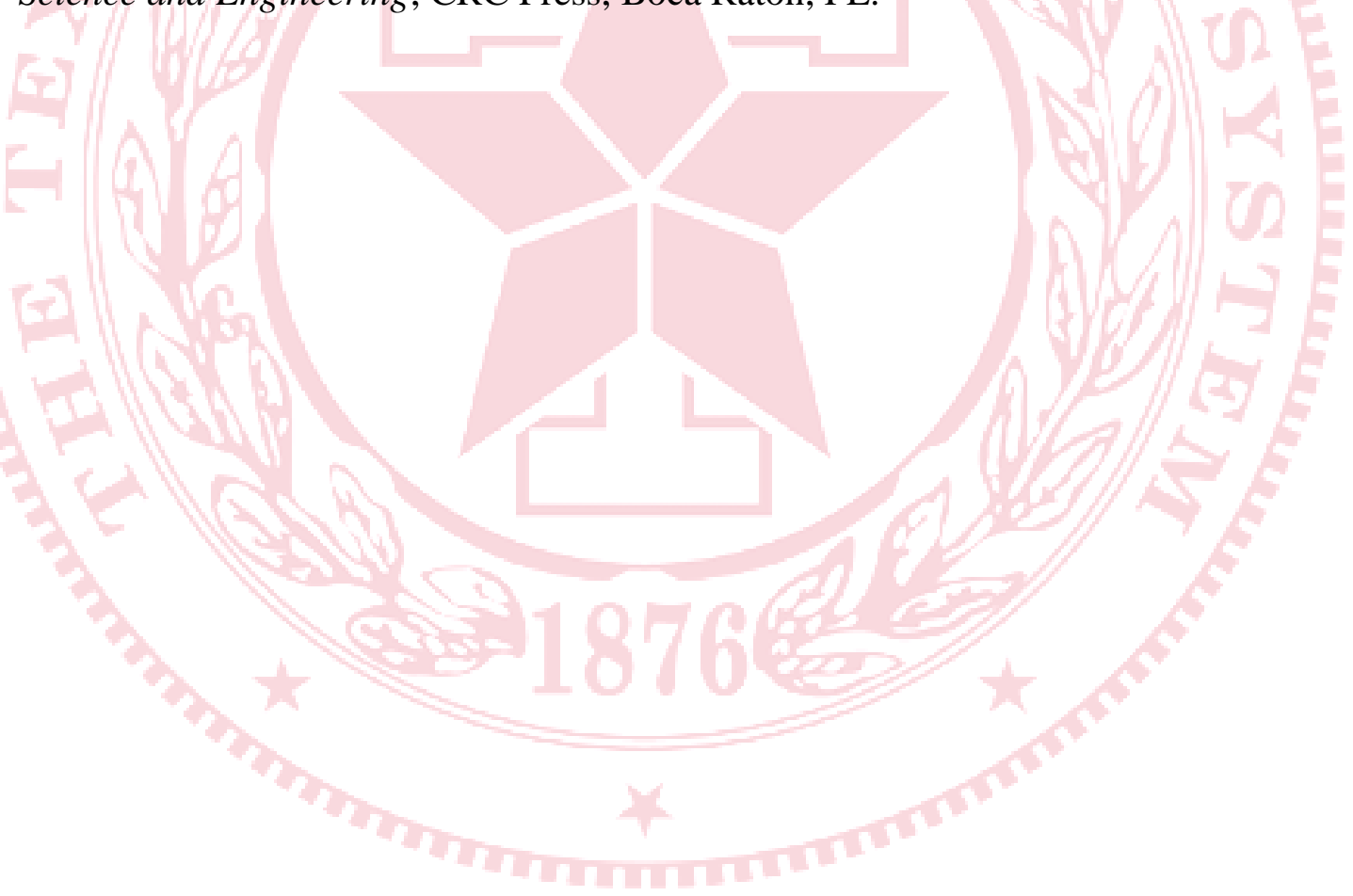




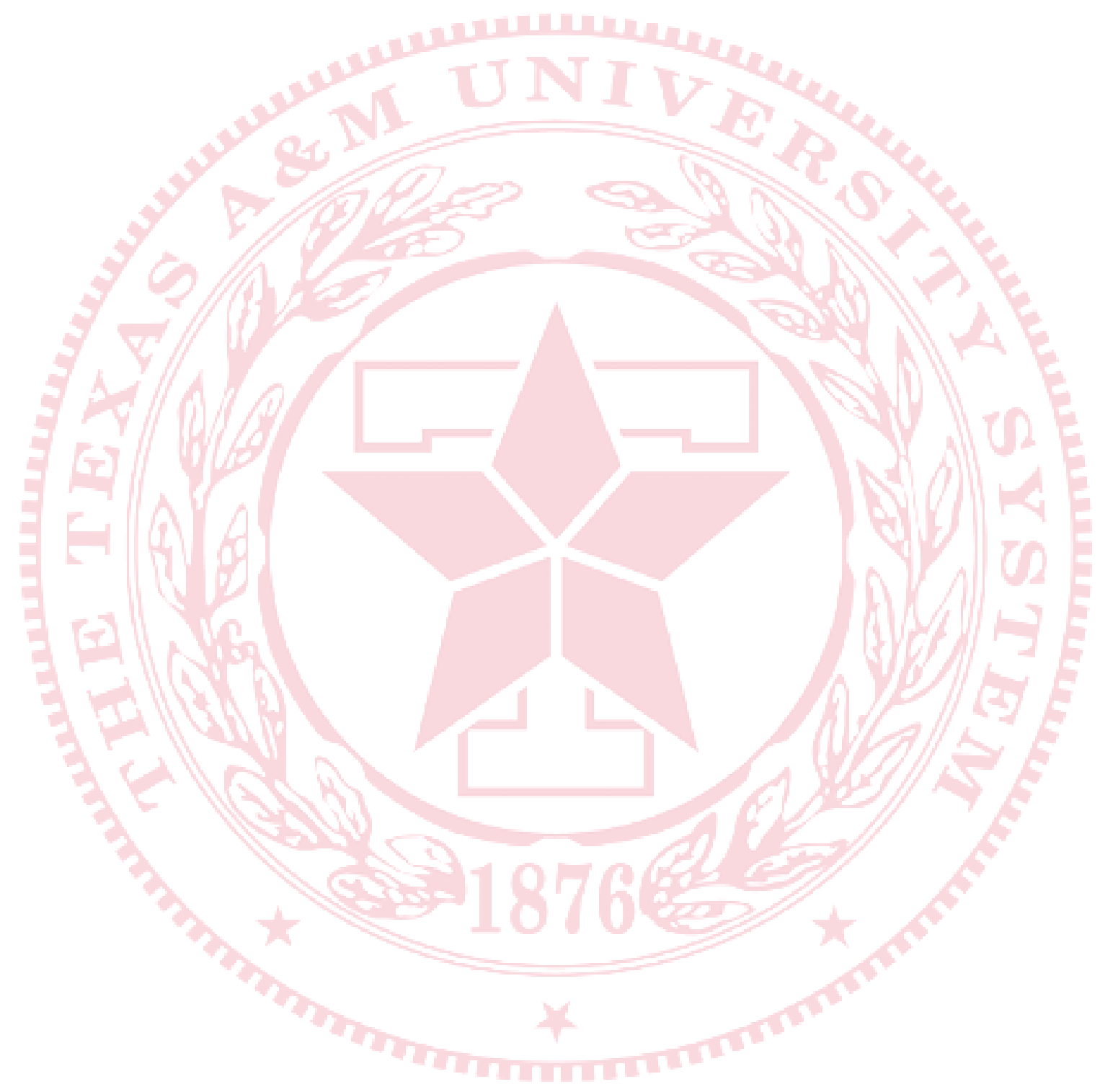




\section{CHAPTER I}

\section{INTRODUCTION AND LITERATURE REVIEW}

\subsection{Motivation}

Global energy demand has greatly increased in recent decades. Since burning fossil fuels emits remarkable amounts of greenhouse gases into the atmosphere, in order to prevent global warming from becoming worse and to reduce emissions of greenhouse gases, clean energy such as solar, wind, and tidal sources is the only solution. However, the efficiencies of these clean energy sources are insufficient to support growing electricity demand, and the costs of the clean energies are very expensive nowadays. Nuclear power plants seem to be the second best solution compared to other energies because of high efficiency, clean energy, and cheap costs. Nuclear reactor designs have been under development for many decades starting from the first generation the nuclear power plants, the research Light Water Reactors (LWRs). The second generation of the nuclear plants is then regarded as commercial LWRs. The pressurized water reactors (PWRs) and the boiling water reactors (BWRs) are the most common designs using the LWR concept. Since light water which is used as the coolant in LWRs is economical and easily obtained, the third and the third-plus generations are focused on improving the performance of the LWRs. The latest nuclear reactor designs, known as High Temperature Gas-cooled Reactors (HTGRs), are motivated by Next Generation Nuclear Plant (NGNP) that encourages researchers in materials and high efficiencies [1-3]. A new configuration that provides additionally passive cooling capability has been designed to HTGRs. Although more cooling configurations and emergency cooling systems are implemented in nuclear plants, the maximum thermal power cannot reach the same level of the fossil plants. Unlike fossil fuel power plants, the maximum heat flux in the nuclear power plants (LWRs and HTGRs) is limited due to the restrictions of material properties, such as boiling point, melting point and thermal conductivity. An enormous heat flux that is even small compared to combustion power plants can fail the fuel cladding structure and cause fuel melting problems, which the releases fission gases [4]. When dealing with light water as the coolant, the large heat fluxes require higher order of attention for the design calculations in order to prevent critical heat flux (CHF) in BWRs or phase changing in PWRs. The CHF condition in BWR is coolant film dryout failure around the fuel rods. After the coolant dries, the zirconium cladding is overheated immediately and starts to react with light water. This accident will finally release hydrogen gas and induces hydrogen explosions because of high temperature and oxygen. Since HTGRs use gas as the coolants, phase changed is not considered. 
However, the failures in the fuel rods (fuel and cladding) are still restricted by the heat fluxes. In other words, the thermal hydraulics study of nuclear power plants is essential in developing new nuclear reactor concepts with alternative coolant, moderator, or fuel. In addition, the study of the simulations for the accident predictions helps understand the design concepts more.

\subsection{Air Ingress Phenomenon}

\subsubsection{Background}

Operating nuclear reactors require designs containing comprehensive cooling systems and external power supplies during accidents, such as emergency cavity cooling system and external electricity generators. The external power generators are used to maintain pumps working in the primary cooling loop during the reactor shutdown. Unlike fossil power plants, nuclear plants continuously release decay heat after the reactor shutdown with approximate $20 \%$ of the previously operating thermal power. Without the cooling pumps, the huge heat fluxes can dry water coolant out in a few minutes and cause CHF problem. Besides, absence of the coolant will result in severe accident, for example, fuel failure and hydrogen explosion. Since HTGRs utilize graphite bricks as the moderators, to avoid oxygen contacting with graphite is compulsory in a very high temperature environment. Although HTGRs have no dryout conditions, any size of breaks can happen everywhere in the reactor vessels and easily destroy them. Oxygen that enters the reactor vessel through the break and contacts with graphite will cause burning and oxidation and further failure the reactors. Air or oxygen intrudes into the reactor through breaks is regarded as the air ingress phenomenon following the loss of coolant accident (LOCA). The behaviors of two different fluids are governed by gravity, so the phenomenon is also defined as gravity currents.

\subsubsection{Previous research reviews}

Gravity currents or otherwise named as density currents, consist of a heavy fluid intruding into a lighter fluid can be observed in large or small scales, such as ocean (salt and fresh water), atmosphere (warm and cold air), laboratory man-made experiments, and other fields. When heavy fluids encounter light fluids, complex turbulent phenomena occur. Many researchers have devoted efforts to understanding flow behaviors. Benjamin started a broad study of gravity currents and proposed a theoretical solution to the two-dimensional steady gravity current in inviscid fluids [5]. In 1972, Simpson introduced a correlation between the depth of the mean cross-section and the mean height of the advancing current through a broad range of Reynolds number from 300 to 10000 in horizontal channels [6]. Simpson and Britter observed KelvinHelmholtz instability and more mixing mechanism generated on the front of the current 
head in horizontal channels [7, 8]. Britter and Linden investigated that using larger slopes in the inclined horizontal channels affected the buoyancy force to greatly reduce the influence caused by frictional force and provided the same wave front head velocity [9]. Huppert and Simpson developed a relationship of the buoyancy force to the inertial force and viscous force for predicting the position of the wave front, which was a function of time with two-dimensional rectangles or axisymmetric disks [10]. Boussinesq approximation was widely studied for the fluid-pairs as water and brine or very low density ratios. Gibson investigated the interactions of shallow and deep ocean waves where the Boussinesq approximation can be adopted. Gibson observed that the estimations of dissipation rate and turbulent velocities might underestimate the spacetime averaging results in short vertical or short horizontal shear layers [11, 12]. Gardner and Crow studied air bubble motion in horizontal channels containing water, and Wilkinson included surface tension effects on bubble motion in the water horizontal ducts $[13,14]$. Rottman and Simpson observed that there were two different phases of the gravity front due to the behavior of the front speed. The velocity of the gravity current is constant after instantaneous release of salt water in the fresh water channel as the initial phase. The velocity decreased as $\mathrm{t}^{-1 / 3}$ when the flow becomes self-similar [15]. Webster investigated the density-driven stratified shear flow of a wind tunnel where the density gradient was created by heating air [16]. In atmosphere and ocean science, Lin $e t$ $a l$. investigated the density stratified flow in a wind channel by heating the ambient air and cooling the lower boundary. Strong turbulence was discovered in the upper part of the rotor [17]. Based on the studies using two uniform streams of air in wind tunnels, Scotti, and Scotti et al. investigated different heated fluids for the Richardson number from 0.07 to 0.76 with the Reynolds number from 30 to 70 [18, 19]. Furthermore, Pao, Lange, and Dickey observed the decaying turbulence in stable stratified flows in horizontal and vertical channels [20-22]. Pao observed that the stratified flow far downstream had a layered structure which was created by the turbulent convection mixed by the mean flow [20]. Lange investigated fluctuation statistics of variety parameters in a horizontal channel and provided a decay law for density variance by approximately $\mathrm{t}^{-\mathrm{r}}$ where $\mathrm{r}$ was from 0.3 to $0.6[21]$. Dickey studied that the turbulent stratified flow with a short decay time in a wind tunnel for a non-dimensional time of 800 was similar to the case with the number of 275 [22]. Dickey observed that the decay rate greatly decreased when the internal gravity waves replaced the turbulent fields [22]. Stillinger et al. studied density-driven stably stratified shear flows for the velocities from steady to $30 \mathrm{~cm} / \mathrm{s}$ with the density ratio from 1.0 to $1.1 \mathrm{~g} / \mathrm{cm}^{3}$ and found a method to maintain arbitrary velocity profiles in each layer in time [23]. Boussinesq approximation is applicable for few percent of density ratios. In 1992, Grobelbauer et al. investigated the propagation of non-Boussinesq fronts with high density ratio from 1 to 20 in the 
exchange flow [24]. Grobelbauer et al. observed that the heavy- and light-fluid front velocities had limitations for the extrapolations to infinite depth and had good predictions from shallow-layer theory [24]. Barnea et al. concluded that the results of wide range of liquid viscosity showed similar amplification rate between the inviscid and the viscous Kelvin-Helmholtz analyses [25]. Barnea et al. observed that the fluid with a large viscosity was appropriate to be modeled with the inviscid Kelvin-Helmholtz theory whereas the low liquid viscosity had a significant discrepancy between the inviscid Kelvin-Helmholtz theory and the viscous Kelvin-Helmholtz theory [25]. Recently, Hartel et al. observed that the free-slip case showed results close to the classical Benjamin theory because the Boussinesq approximation was adopted for the slight density differences [26]. Shin et al. showed that dissipation rate was insignificant with high Reynolds numbers and developed a new theory for predicting gravity current velocity [27]. Gu and Guo studied Kelvin-Helmholtz criterion of the interfacial wave instability for horizontal and near horizontal pipes [28]. Gu and Guo observed that if the pipe inclination is greater than $0.4^{\circ}$, the critical heavy fluid height is insensitive to pipe inclination [28]. Gu and Guo developed a fitting curve of wave front speed versus wave front height in pipe flow [28]. Stretch et al. observed the mixing efficiency in stratified flow where irreversible mixing mechanism appeared for turbulent kinetic energy converting back to potential energy. According to the simulation results obtained from DNS and rapid distortion theory, the mixing efficiency increased while initial Richardson numbers were small, but the efficiency remained constant for larger Richardson numbers [29]. Moreover, Lowe et al. developed a hydraulic model with twolayer fluids for the experiments with the density ratios from 0.61 to 1 [30]. Lowe et al. found that it was more unsteady for the heavy fluid than the light fluid at the interface [30]. Good agreements were obtained between experimental data and two-dimensional simulations researched by Birman et al. who observed that light currents followed Boussinesq level but heavy currents behaved as dissipative gravity currents [31].

\subsubsection{Problem description}

NGNP organization encourages research in Very High Temperature Gas-cooled

Reactors (VHGRs) under normal operation and accident scenarios such as air-ingress phenomena [32]. The air-ingress phenomenon is the accident scenario happening during a LOCA where the pressure inside the operating system decreases dramatically [33, 34]. Any size of the breaks that occurs at the co-axial pipe connecting the reactor vessel and the heat exchanger will result in a LOCA. The double ended guillotine break is considered as the worst condition happening in the accident even though this break is practically impossible. When the coolant fluid escapes from the reactor vessel and the pressure balances between the reactor vessel and the surrounding environment, the air 
(with oxygen) in the cavity intruding into the reactor vessel, especially the support structure of the lower plenum which is made from graphite, will cause graphite oxidation and burning as described in Section 1.2.1 $[33,35]$. In this problem, the focus is on the behavior of the stratified flows with different fluid pairs in the air-ingress process that is considered as gravity driven flows. Idaho National Laboratory (INL), under the U.S. Department of Energy (DOE), has organized many participants recently researching in this topic for VHTRs which has been researched since 1950s [1-3]. Oh, Kim, and many other participants studied air-ingress stratified flows in rectangular channels and pipes $[33,36,37]$. In this research, the prototype designed from General Atomic was used to study gravity currents inside the coaxial pipe with assuming a double ended guillotine break [38]. STAR-CCM+ will be used to obtain simulation results which will be validated with experimental data done by Hartley [39].

\subsection{Objective}

The air-ingress problem following a LOCA has been regarded as a potential severe accident in HTGRs [33]. The concentrations of oxygen gas in all scenarios with different the break sizes directly affect the graphite oxidation conditions. Even though the double ended guillotine break is practically impossible, it is assumed as the most disastrous condition because of its large break size. In order to predict and validate this shortly transient scenario, the gravity-driven stratified flow following an air-ingress phenomenon is investigated in this research. Helium as the coolant in HTGRs and air surrounding the environment are two fluids in the real accident. A fluid similarity scaling is provided to mimic the real accident because helium and air is difficult to dye. Most of the previous research studies are done on rectangular channels, ducts, or pipes containing salt and fresh water. The study of how two fluids behave in coaxial pipes becomes a new motivation in this research since the research in coaxial pipe is a new topic for gravity-driven stratified flows. A liquid-liquid scenario and a gas-gas case will be simulated in this research.

Currently, CFD methodology has become a major tool for studying and predicting fluid hydrodynamics. CFD will be used to model all scenarios because of the conveniently obtaining results, such as visual graphic interface and various packages of turbulent modeling. Different commercial CFD codes e.g., STAR-CCM+ and ANSYS $\mathrm{CFX}$, will be used to obtain simulation results for comparing and validating with experimental data [40-42]. MATLAB were used for the post analyses [43]. Besides, the mesh size sensitivity studies are performed to obtain numerical convergence with error analysis. Moreover, wavelet analysis and proper orthogonal decomposition (POD) were applied to characterize turbulence [44-47]. 


\section{CHAPTER II}

\section{DESCRIPTION OF EXPERIMENTAL FACILITY}

\subsection{Scaling Process of Experiments}

The GT-MHR design shown in Figure 2 includes the prismatic core with a lower plenum, the co-annular duct, and the heat exchanger and the turbine. As the arrows presented in Figure 2, hot helium from the reactor core goes through the hot duct of the coaxial pipes (inner pipe) and exchanges heat in the turbine and the electricity generator. Then, cold helium compressed in the compressor moves back to the core vessel via the outer pipe as the coolant that removes heat from the fuels and finally moves toward the heat exchanger as a close loop. Any break that occurs in the pipe or the reactor vessel will cause a loss of coolant accident (LOCA) and will eventually result in the air ingress phenomenon. In order to validate that the scale down to laboratory had the same VHTR conditions during a LOCA, the same fluid similarity between the real condition and the laboratory facility was performed [39]. Moreover, the scaling process was performed to represent the experiments for studying the gravity-driven stratified flow phenomenon and to mimic the real condition during the accident in the VHTRs. It is clear that the airingress phenomenon under prototype conditions has the following parameters: $\mathrm{Fr}=0.33$ and $\mathrm{Ri}=0.68$ [39]. The Froude number and the Richardson number are two common dimensionless parameters for studying stratified flows.

The Froude number is defined as the ratio of inertial force to gravitational force, and the Richardson number is the ratio of buoyancy force to inertial force. Boussinesq approximation assumes that the inertia difference is unimportant and the gravity effects are strong to let specific weight be significant between two fluids; as a result, the Boussinesq approximation is applied to the Froude number and Richardson number to study density difference of the fluid pairs. In this study, the Froude number and Richardson number were calculated from Eq. 2-1 and Eq. 2-2.

$$
\begin{aligned}
& F r=\frac{\text { inertial forces }}{\text { gravitational forces }}=\frac{U}{\sqrt{g h}} \\
& R i=\frac{\text { buoyancy forces }}{\text { inertial forces }}=\frac{g^{\prime} h}{U^{2}}=\frac{g \frac{\rho_{\text {heavy }}-\rho_{\text {light }}}{\rho_{\text {average }}}}{U^{2}}
\end{aligned}
$$

where $\mathrm{g}$ is gravity, g' is reduced gravity, $\mathrm{h}$ is wave front height, and $\mathrm{U}$ is wave front speed. The Benjamin theory was used for the gravity current front velocity in the scaling analysis [5]. 


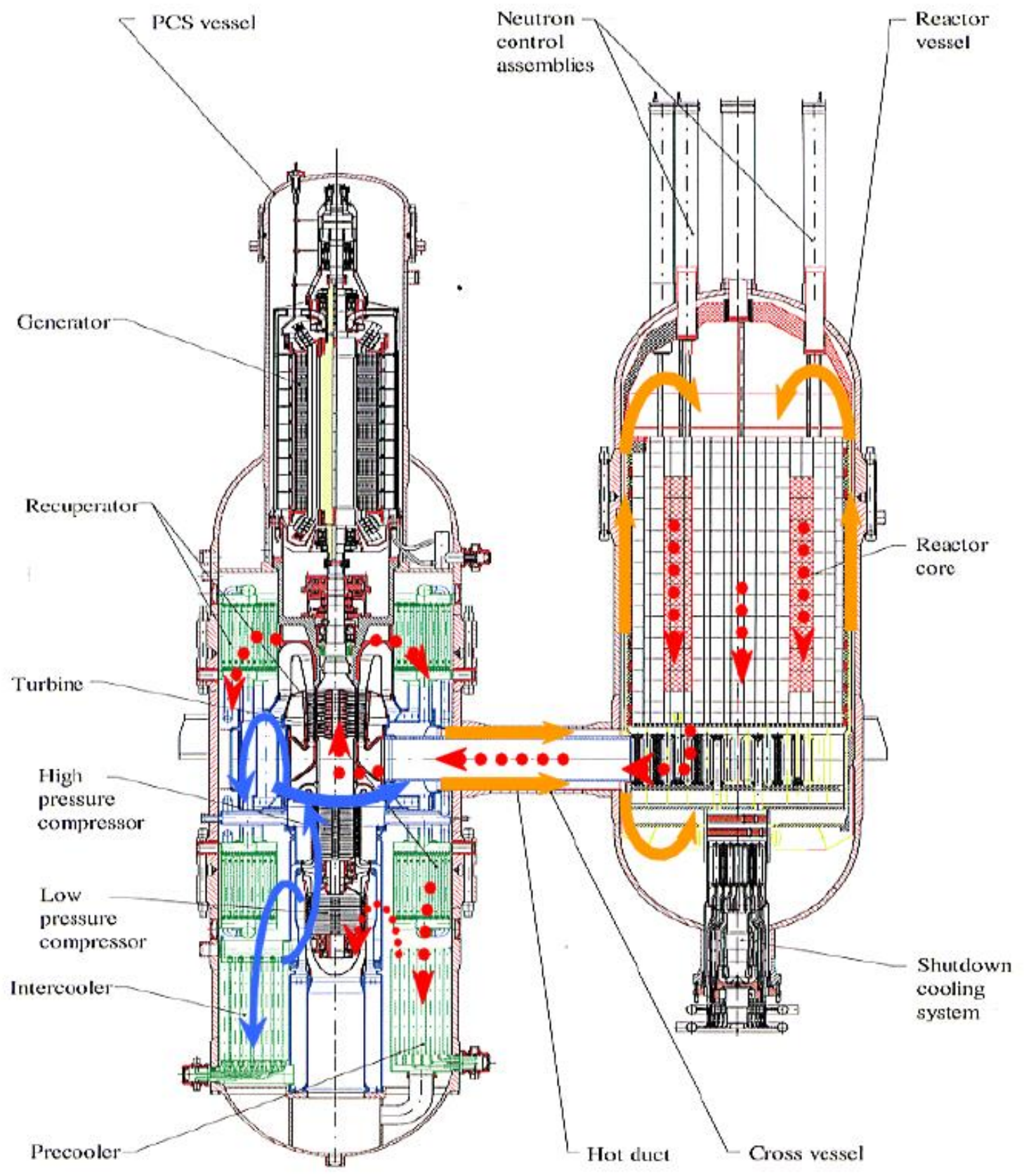

Figure 2. Design configuration of the GT-MHR [38]. 
Table 1 lists the comparisons of the fluid similarity in the real and the experimental conditions. In this study, two fluid pairs, helium-SF 6 and water-brine pairs, are used for the experiments. Helium and $\mathrm{SF}_{6}$ were chosen for this problem because the dimensionless numbers were the same as those occurring in the actual air ingress scenario of the reactor (helium and air). As a result, using the fluid pairs (helium and $\mathrm{SF}_{6}$ ) in the experiment and the simulation will allow the gravity current front velocity to be the same as that in the real air ingress condition in the reactor. Additionally, $\mathrm{SF}_{6}$ is easier to dye than air, so $\mathrm{SF}_{6}$ was used in the experiment. Although the dimensionless number of water and brine pairs does not match the real condition, the liquid-liquid case is still utilized because of the good visualization. Moreover, the water-brine case is much easier to capture important phenomena than the gas-gas scenario because it is more difficult to dye gases.

Table 1. Lists of fluid pairs used for the reactor similarity.

\begin{tabular}{l|lll}
\hline Fluid Pair & Froude Number & Richardson Number & Density Ratio \\
\hline Helium-Air & 0.33 & 0.68 & 0.14 \\
Water-Brine & 0.43 & 4.89 & 0.88 \\
Helium-SF $_{6}$ & 0.33 & 0.68 & 0.14 \\
\hline
\end{tabular}

\subsection{Geometry and Dimensions of Experimental Facility}

The experimental prototype established at Texas A\&M University is a simplified module with an approximate 1:20 scale of the original GT-MHR design [38]. The prototype is consisted of two identical fluid tanks, a knife gate valve, and a coaxial connecting pipe. The dimensions of the experimental facility are shown in Figure 3. It must be mentioned that the diameters of the inner and the outer pipes are $0.066 \mathrm{~m}$ and $0.1 \mathrm{~m}$, respectively. Two different lengths of the co-axial pipe, $0.38 \mathrm{~m}$ and $1 \mathrm{~m}$, are used to characterize the pipe break locations in order to study the effects of the break locations. Fluids are filled from the top of the tanks, and the moving valve will be moved in the direction toward the reader in the experiments. 


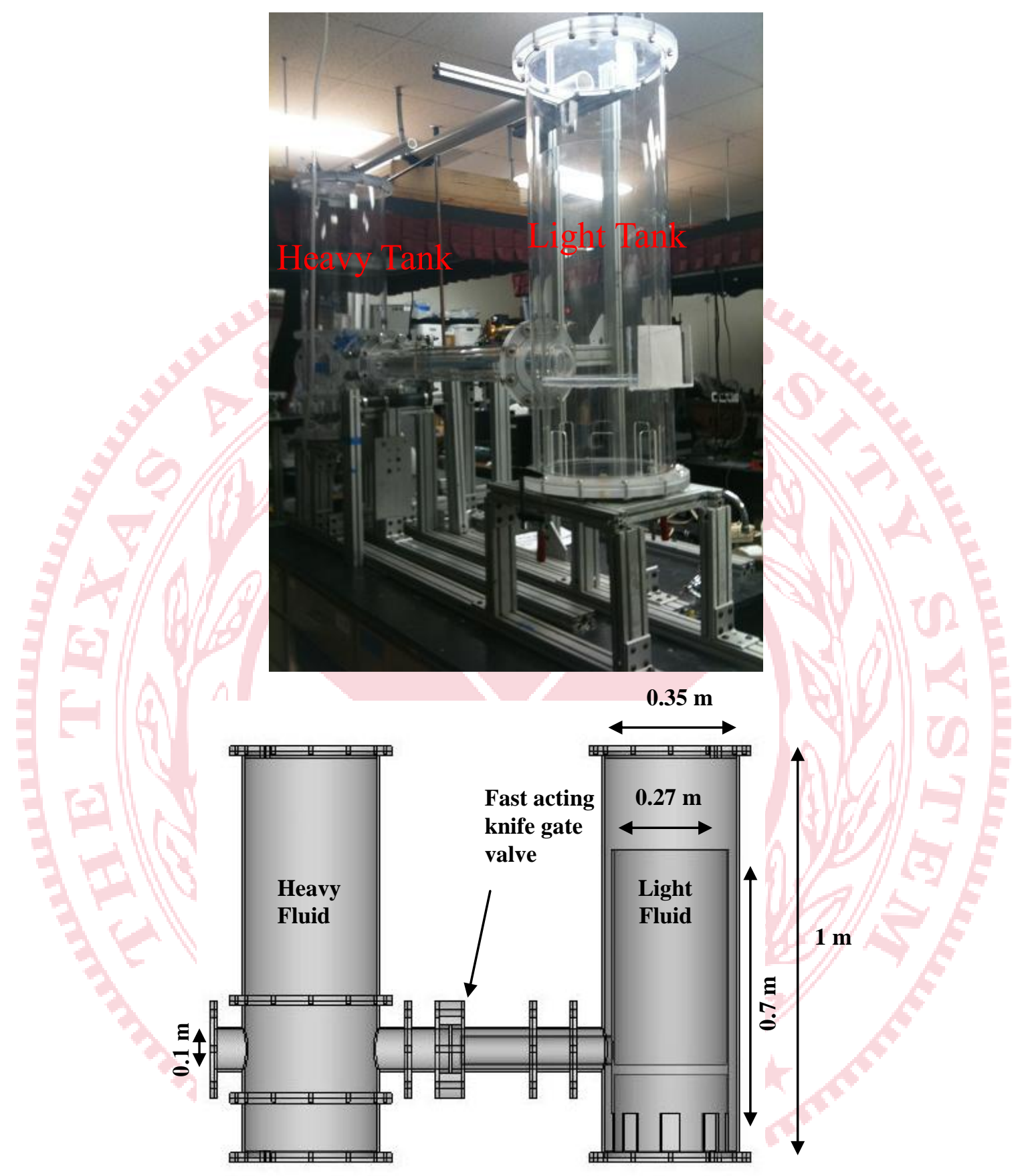

Figure 3. Laboratory experimental facility at Texas A\&M University. 


\subsection{Initial and Boundary Conditions in Experiments}

Two fluids were filled to the tanks and perfectly separated by the valve located at the connecting pipe as the initial condition. For the boundary condition, the two fluids were measured at the same pressure and at steady state. After the fluids reached equilibrium, the valve was suddenly open. During the experiments, the total physical time to open the valve was $0.534 \mathrm{~s}$ in the direction toward the reader (Figure 3). Since the valve moved within an extremely short time, it was assumed that the valve moved with a constant speed for the simulation. In the water-brine case, the brine solution is dyed for visualizations. For the helum- $\mathrm{SF}_{6}$ scenario, it is difficult to dye $\mathrm{SF}_{6}$, so the particles that were applied to $\mathrm{SF}_{6}$ were irradiated by the laser lights. To observe the air ingress phenomenon, 2 (short-pipe) or 3 (long-pipe) high speed cameras were used to capture transient flow behaviors.

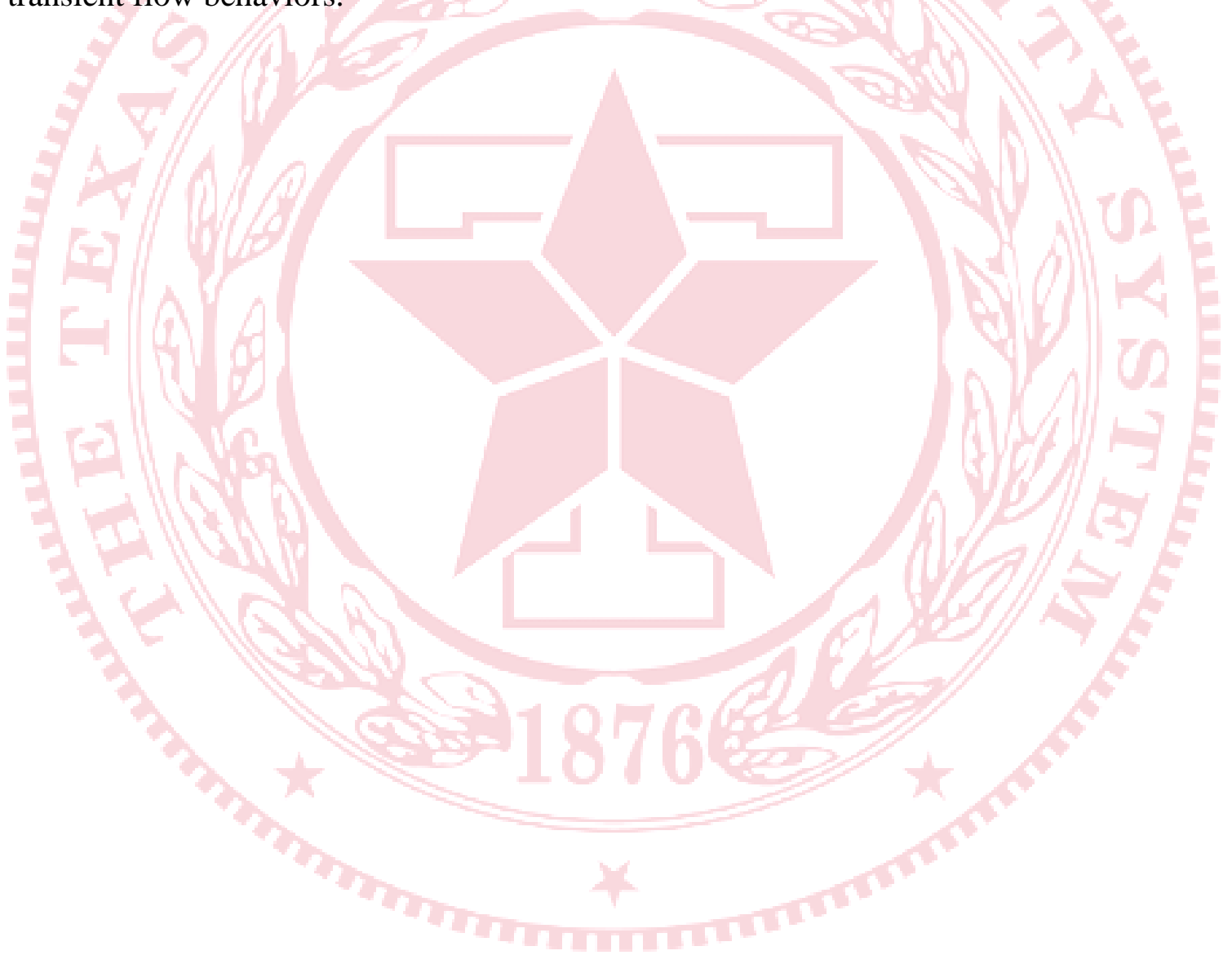




\section{CHAPTER III}

\section{DESCRIPTION OF THE CFD MODEL DEVELOPED}

Many issues can significantly influence CFD simulation results, such as mesh type and sizes, turbulent models, near-wall treatments, and boundary conditions. Usually, a coarse mesh size predicts unstable calculations; as a consequence, the mesh size independent study is used to obtain calculations with high confidence. Besides, understanding appropriate assumptions and models before implementation in the simulation is extremely required; otherwise, the results are inaccurate or meaningless. Wrong boundary conditions result in incorrect answers even though the simulations are converged; therefore, results are more meaningful with proper boundary conditions. Currently, it is not possible to directly solve Navier-Stokes equations without any turbulence models, such as RANS and LES, because it consumes enormous computational resources to solve all details. Selecting a suitable turbulent model, using reasonable boundary conditions, and using enough fine mesh size are the optimizations for CFD.

STAR-CCM+5.02 was the software used in all air ingress simulations. The dimensions of the geometry, the grid sizes of meshes, the selections of the turbulent models, the initial and boundary conditions, the working fluids, and the technique of obtaining transient results were discussed in the following sub-sections.

\subsection{Simulation Geometry and Mesh Grids}

Since the study is to mimic the heavy fluid intruding into the reactor, the experimental configuration has been modified to a simple geometry that contains two tanks, one valve, and one co-annular duct with two lengths $(0.38 \mathrm{~m}$ and $1 \mathrm{~m})$ as illustrated in Figure 4. Unlike the experimental geometry (Figure 3), the heavy fluid was placed in the right tank, and the light fluid was located in the left tank (Figure 4). Three different mesh sizes of $1.5 \mathrm{~mm}, 1.0 \mathrm{~mm}$, and $0.7 \mathrm{~mm}$ shown in Figure 5 were used for the mesh size sensitivity study and also for the computational uncertainty study [50]. Because the focus was on the flow behaviors inside the coaxial pipe, the finer mesh size was only applied to the pipe region in order to obtain more details.

In the other parts of the flow domain, $2.5 \mathrm{~mm}$ surface size was set for all cases to mesh grids to reduce computational needs. For example, in the case has with the highest number of the volume cells, the $2.5 \mathrm{~mm}$ surface mesh size was set everywhere and the $0.7 \mathrm{~mm}$ surface mesh size was used on the pipe. The polyhedral volume mesh was applied to generate volume cells. Mesh size sensitivity study was only performed on the 
short-pipe geometry in which cases with $1.5 \mathrm{~mm}, 1.0 \mathrm{~mm}$, and $0.7 \mathrm{~mm}$ surface sizes contained approximately 5.4M, 10.6M, and 23.5M volume cells, respectively [50]. For the long-pipe geometry, only the $0.7 \mathrm{~mm}$ surface size was utilized, and the total polyhedral volume cells were approximate 40M volume cells [50]. Table 2 lists the information of the geometries and the mesh sizes with corresponding amount of volume cells.

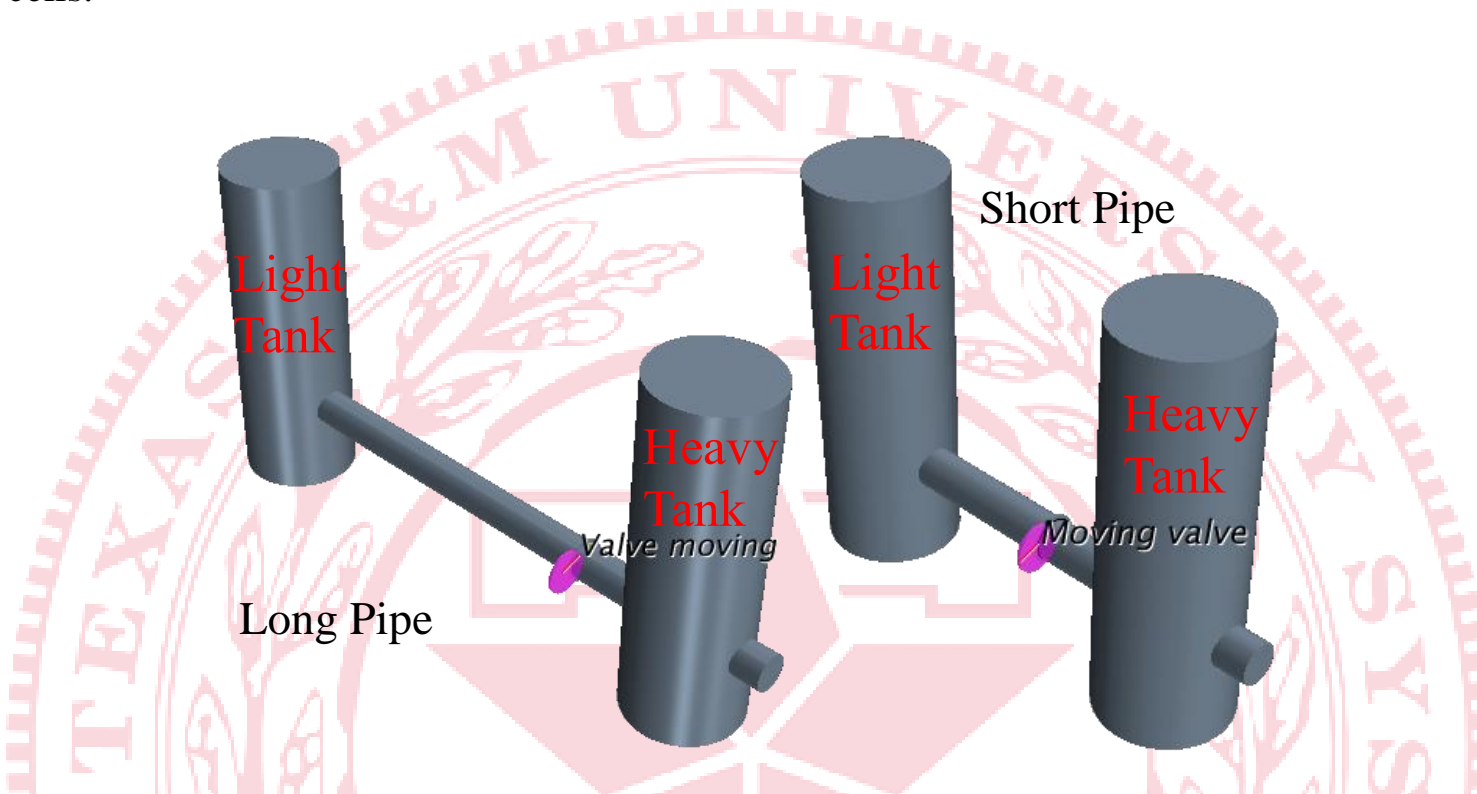

Figure 4. Geometry representation in the simulations: tanks, coaxial pipe, and valve. 


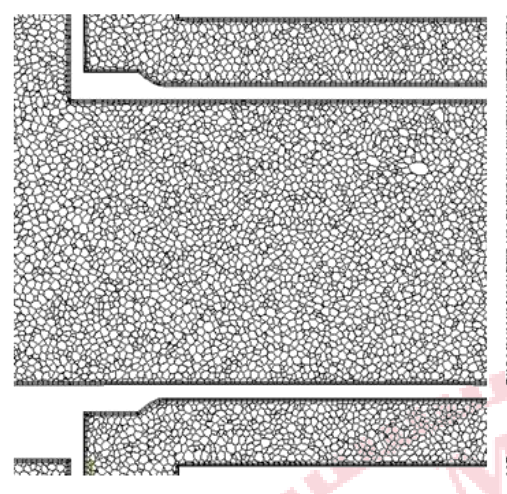

$1.5 \mathrm{~mm}$
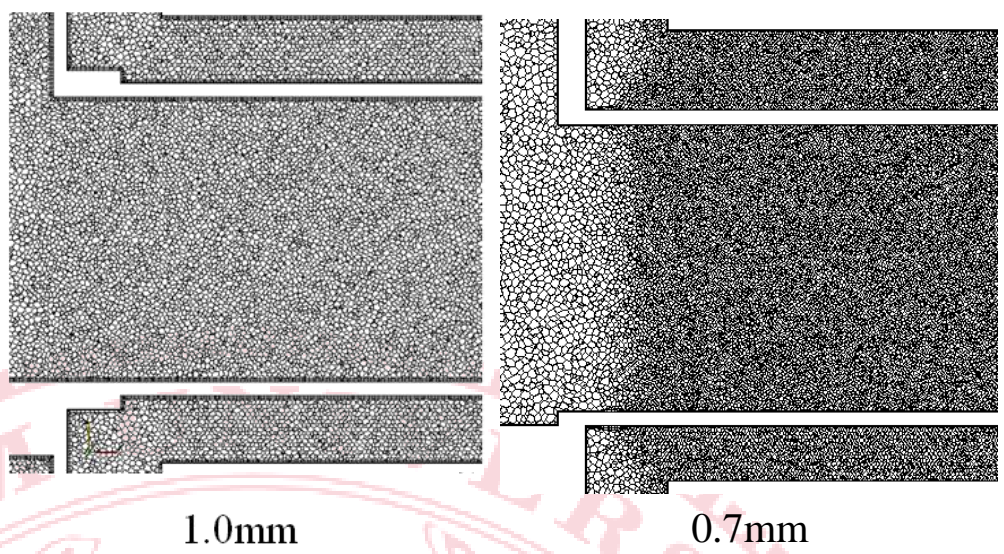

Figure 5. Mesh presentations at the coaxial pipes in the simulations. Polyhedral volume cells were applied to generate meshes.

Table 2. Lists of mesh size and number of volume cells.

\begin{tabular}{llll}
\hline Grid Number & Cell Size & Number of Cells & Geometry \\
\hline 0 & $0.7 \mathrm{~mm}$ & $39.8 \mathrm{M}$ & Long \\
$\overrightarrow{1}$ & $0.7 \mathrm{~mm}$ & $23.5 \mathrm{M}$ & Short \\
2 & $1.0 \mathrm{~mm}$ & $10.6 \mathrm{M}$ & Short \\
3 & $1.5 \mathrm{~mm}$ & $5.4 \mathrm{M}$ & Short \\
\hline
\end{tabular}

\subsection{Turbulent Models Selections}

The air-ingress problem was a transient accident, so the implicit solver was used to solve the governing equations. The unsteady RANS (URANS) model and the LES approach in STAR-CCM+ were two different concepts of turbulent models applied to this study in order to compare the results [40]. The RANS model is based on the timeaveraged properties of the fluid flow, which are divided into two parts (i.e., the average value and the fluctuating part). On the other hand, the LES model is the concept based on the spatial average that depends on the mesh size as the filter, which separates fluid quantities into the spatial average term and the fluctuating term [49]. As described in Section 3.3.3, the unresolved limit of the filter depends on the grid size. The comparison between the averaging quantities (URANS) and the instantaneous results (LES) was described in CHAPTER VI. The unsteady Realizable k- $\varepsilon$ two-layer model was selected to solve the average quantities at each time step in this study. The instantaneous quantities in the LES model were directly solved in large scales, and the unresolved quantities were modeled in small scales. As a result, the WALE SGS model was applied 
to compute the unresolved turbulent scales for the transient problem. In order to simulate the turbulent effects in the near-wall region, all $y+$ wall treatments were selected for both turbulent models.

\subsection{Description of Simulations}

As described in Section 2.1.3, the tanks were filled with two fluids and were blocked by the valve which was assumed to open in $0.534 \mathrm{~s}$ with a constant speed. In some scenarios, the valve was considered fully open as the initial condition. For example, the fully open valve was implemented to the mesh sensitivity study. This assumption is acceptable because the purpose of the sensitivity study is to understand the mesh size convergence. Three different mesh sizes of $1.5 \mathrm{~mm}, 1.0 \mathrm{~mm}$, and $0.7 \mathrm{~mm}$ were applied for the grid size independent study where the simulations utilized $\mathrm{He}_{-} \mathrm{SF}_{6}$ as the fluid pairs with the Realizable k- $\varepsilon$ model, the short pipe geometry, and the fully open valve condition. These simulations as describe in Section 4.1 .1 contain $5.4 \mathrm{M}, 10.6 \mathrm{M}$, and $23.5 \mathrm{M}$ cells [50]. The time step was set to one millisecond (1ms) in all simulations. The gravity influence was set in the negative y direction to include gravity effects.

For the valve moving test, the Unsteady Realizable k- $\varepsilon$ model was used because the Realizable k- $\varepsilon$ model was the only model applied to the fully open valve scenarios. The LES model was utilized in the pipe length study. Using the different pipe length was to investigate the effects of the break location on the coaxial pipe. In order to collect simulation results, two horizontal and six vertical line probes were included in the simulation. Two horizontal probes, line-probe HU and line-probe HB, were aligned with the walls of the inner and the outer pipes, respectively (Figure 6). The vertical probes were located at equal distances from the entrance of the tank to the valve as presented in Figure 6. Besides, an axial plane cross-section of the inner pipe was created to obtain results shown in Figure 7. The resolutions of the horizontal and vertical axes were identical to the mesh size that was set to $0.7 \mathrm{~mm}$. Therefore, the axial plane cross-section that was equally discretized with $0.7 \mathrm{~mm}$ contained total 1471 by 101 cells. Four transverse plane sections were created to store the transient graphs in each time step for the short-pipe simulations as shown in Figure 8. As presented in Figure 9, seven transverse plane sections were used for the long-pipe simulations. The additional transverse plane section was illustrated in Figure 10. The first transverse plane section in both short-pipe and long-pipe cases located at the valve. Figure 11 gives an example of one axial plane section at the time step of 0.567 second. The simulation results in RANS model were compared with the efforts in LES approaches. 


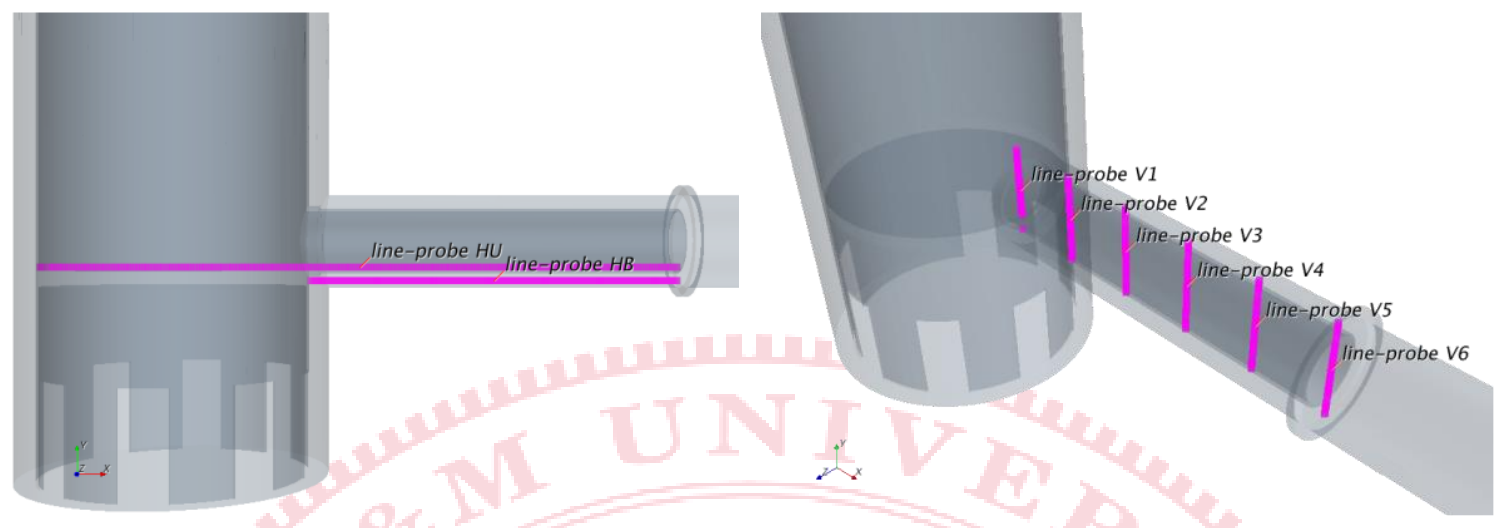

Figure 6. The horizontal and vertical line probes to record transient results.

\section{presentation grid}

Figure 7. An axial plane cross-section presentation uses to obtain results. The resolutions in horizontal and vertical directions are 1470 and 101, respectively. 


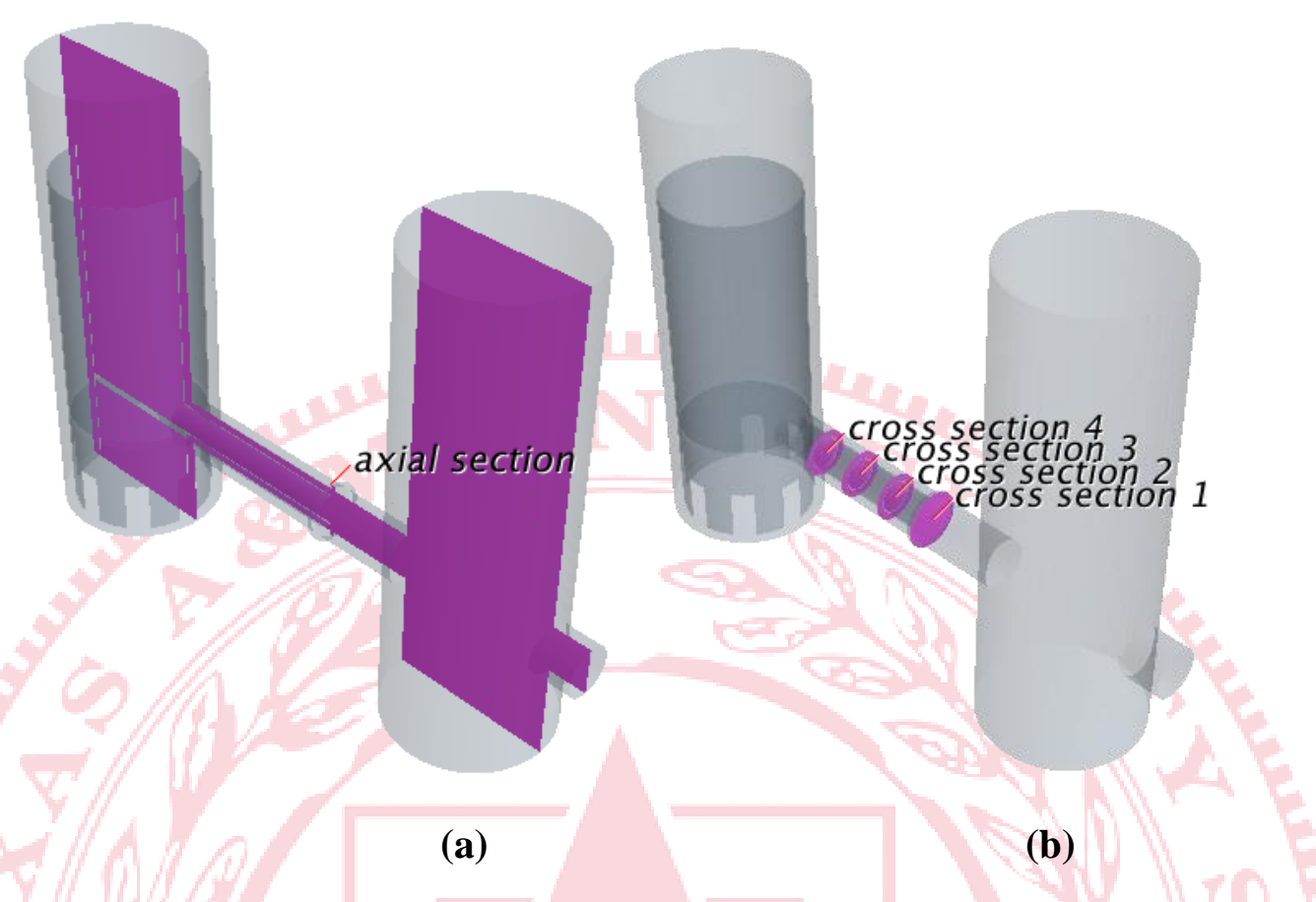

Figure 8. The plane cross-sections used in the short-pipe scenario in STAR-CCM+. (a) one axial cross-section, (b) four transverse cross-sections.

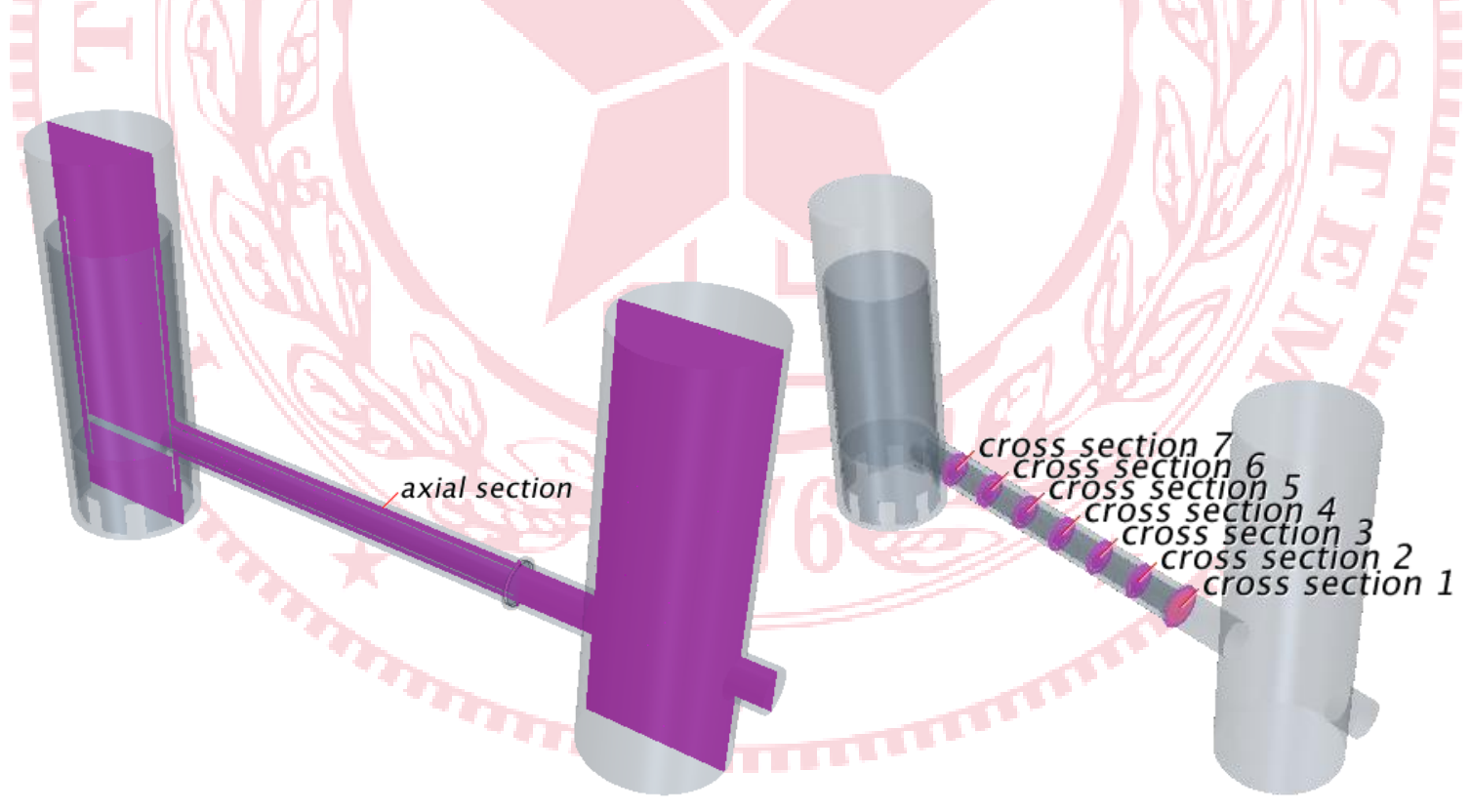

(a)

(b)

Figure 9. The plane cross-sections used in the long-pipe scenario in STAR-CCM+. (a) one axial cross-section, (b) seven transverse cross-sections. 


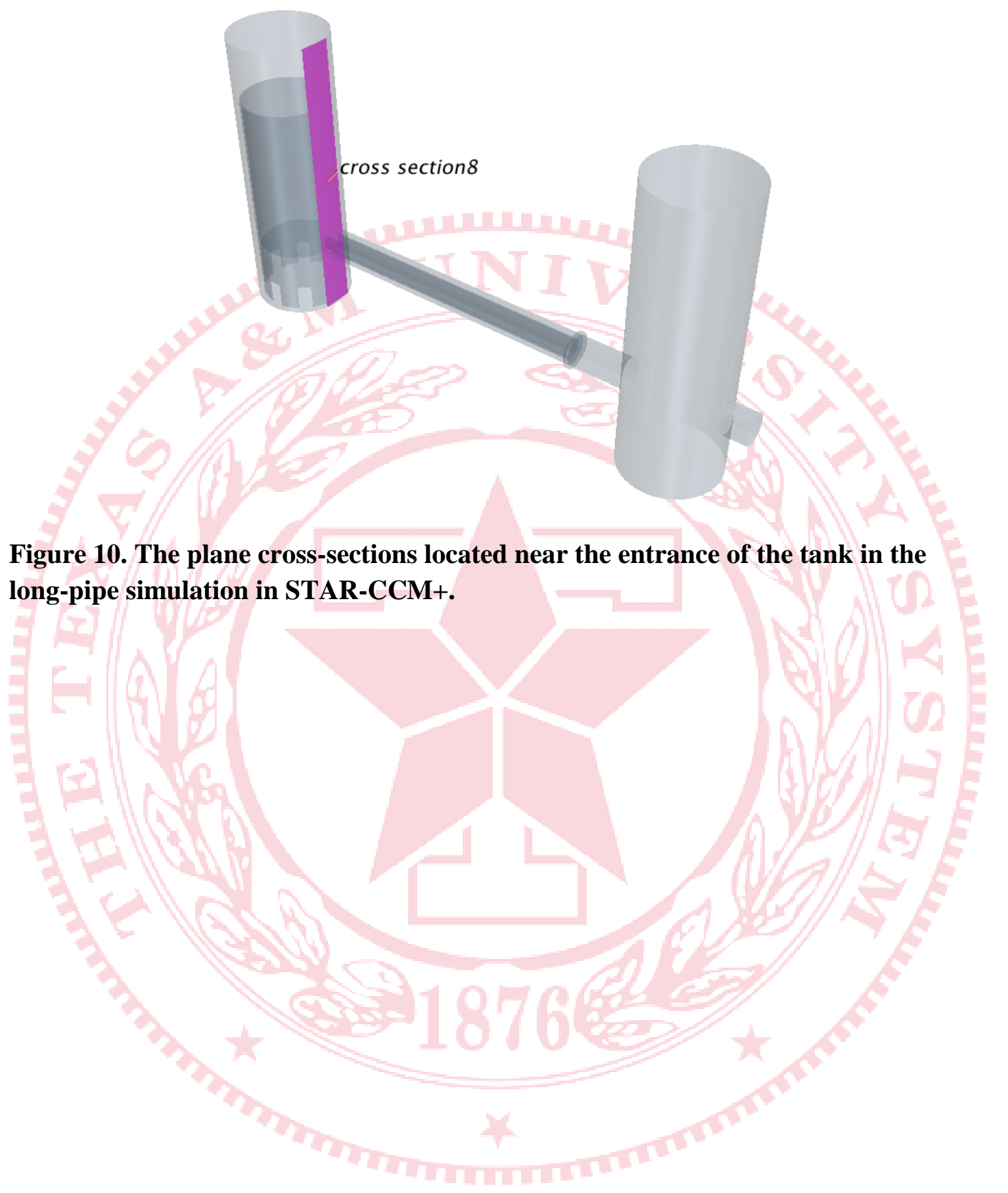




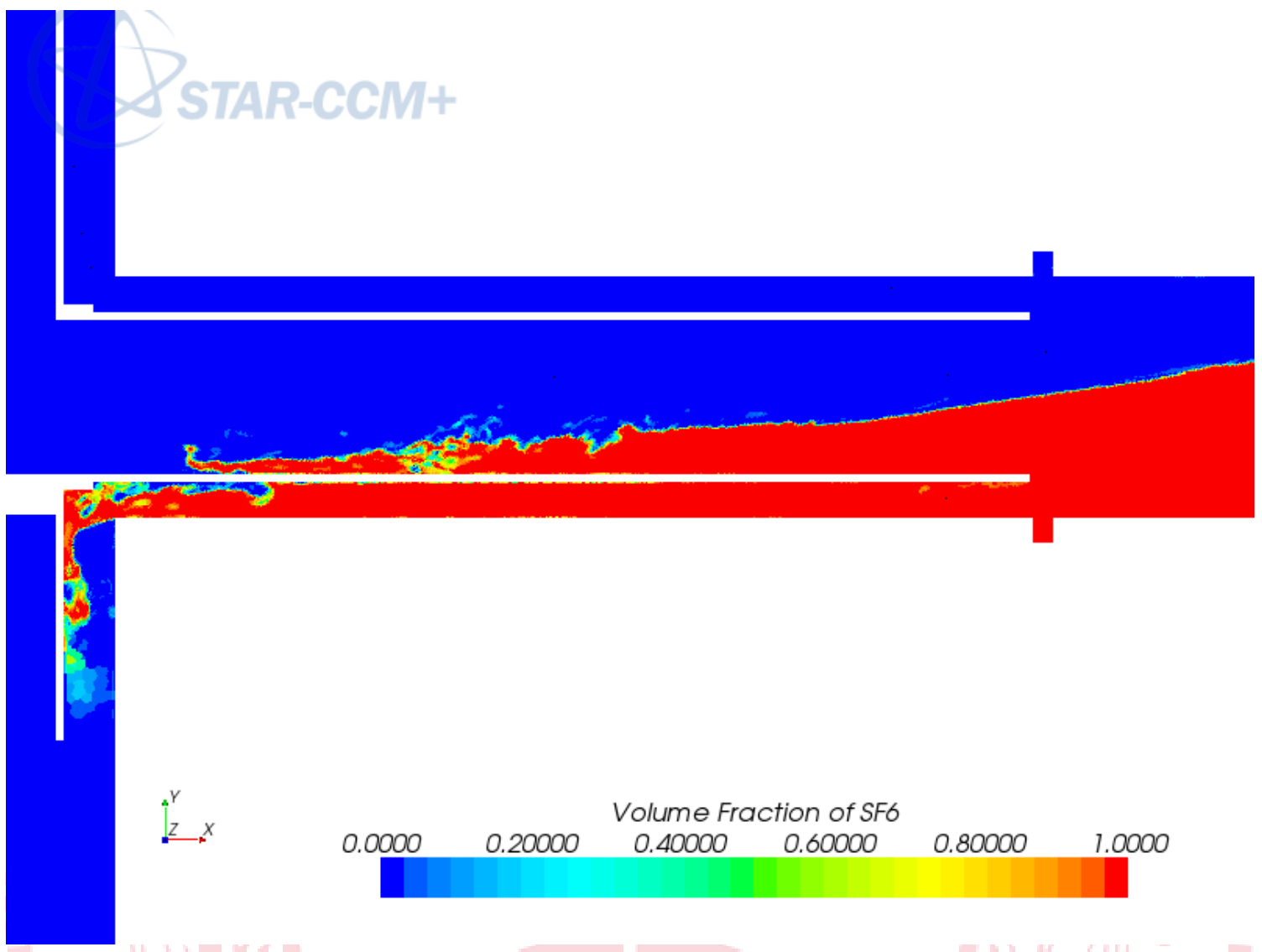

Figure 11. Example of the $\mathrm{He}_{-} \mathrm{SF}_{6}$ short pipe simulation with $0.7 \mathrm{~mm}$ mesh size and LES modeling at 0.567 second. 


\section{CHAPTER IV}

\section{ANALYZING METHODOLOGY}

\subsection{Turbulent Analyses Methods}

Comparing simulation results with experimental data is the most convenient way to validate and verify CFD efforts. Besides, the spectral analysis is used to study CFD simulations. Fast Fourier Transform (FFT) is the command tool for analyzing datasets in the spatial or the temporal domains. Furthermore, FFT can be used to study Kolmogorov's hypothesis where the fluctuating velocities are required to create a turbulent kinetic energy spectrum [48]. If a scenario is a strong transient phenomenon that contains more information of velocity evolution rather than the turbulence cascading energy spectrum, the study of Kolmogorov's hypothesis will be inappropriate [48]. Therefore, it was difficult to create turbulent kinetic energy spectra for the air ingress phenomenon; instead, the energy spectra of the flow evolution were compared in URNAS and LES models. Moreover, Fourier analysis was applied to the OECD rod bundle study for analyzing turbulent kinetic energy spectra that was available with respect to Kolmogorov's hypothesis [48]. Since the OECD study was a quasi-steady state problem, it had no strong effects of the flow evolution. Thus, the turbulent kinetic spectra were studied. Before comparing with Kolmogorov's hypothesis, a line-probe was used to record fluctuating velocities that were transformed by FFT to calculate turbulent kinetic energy in spectral domain. Then, the magnitudes of the energy were calculated from the velocities of FFT results and their conjugates value. The discretization of the line-probe and the amounts of samples were utilized to calculate the corresponding wavenumbers. Subsequently, a new spectral analysis method, wavelet transform has been well developed in the past few decades. The wavelet analysis was used in the research to overcome the deficiencies of FFT, such as solving time and frequency domains simultaneously. In addition, the difference between wavelet transform and FFT is that the wavelets are localized in time and frequency domains but FFT is only localized in frequency domain [44]. Also, FFT is composed of sine and cosine functions, but wavelet transform can have infinity mother wavelets bases. Another analysis method named proper orthogonal decomposition (POD) was implemented for the simulation results because it is advantageous to filter out small signals. POD method is widely used to experimental results to study flow features and invisible flow patterns. The original signals are decomposed to eigenvalues and eigenvectors in the POD. Then, the signal is

reconstructed by selecting different numbers of the eigenvalues, so the small signals are 
filtered out. The concept of POD method is similar to LES approaches. Wavelet analysis and the POD method were discussed more in the following sub-sections.

\subsubsection{Wavelet analysis method}

Spectral analyses were implemented to study the simulation results based on the temporal or spatial domains that were dependent on the sources of the results. For example, if the results are recorded at a certain time step, the analysis will be a wavenumber analysis. If the dataset is from a time series, the analysis will result in a frequency analysis. FFT and wavelet analyses are both useful for spatial and frequency analyses. Although Fourier analysis is convenient and simple to use, unfortunately, it can be applied to only one domain (space-wavenumber or time-frequency) at a time. In order to overcome the defects, wavelet analysis is used to characterize turbulence. Wavelet transform was applied for analyzing results in this research because it can handle results of high order dimensions simultaneously. Wavelet can provide more temporal and local details than FFT and can deal with rapidly changing signals [44].

For example, if the function $\psi$ satisfies Eq. 4-1 and Eq. 4-2, $\psi$ is considered as the mother wavelet.

$$
\begin{aligned}
& \int_{-\infty}^{\infty}|\Psi(t)|^{2} d t<\infty \\
& C_{\psi}=2 \pi \int_{-\infty}^{\infty} \frac{|\Psi(\omega)|^{2}}{|\omega|} d \omega<\infty
\end{aligned}
$$

where $\Psi$ is the Fourier transform of $\Psi$. Eq. 4-1 shows the finite energy condition. Eq. 42 give that $\Psi(0)=0$ if $\Psi(\omega)$ is smooth. Then, the wavelet transform of a real signal is given by Eq. 4-3.

$$
S(b, \mathrm{a})=\frac{1}{\sqrt{a}} \int_{-\infty}^{\infty} \psi^{\prime}\left(\frac{t-b}{a}\right) s(t) d t
$$

where $\psi^{\prime}$ denotes the complex conjugate of $\psi$. The parameters $b$ and a are the time shift and the scale of the analyzing wavelet, respectively. Assuming $\psi_{a, b}(t)$ is defined as Eq. 4-4.

$$
\psi_{a, b}(t)=\frac{1}{\sqrt{a}} \psi\left(\frac{t-b}{a}\right)
$$

Here, the wavelet transform of the real signal becomes as:

$$
S(b, \mathrm{a})=\int_{-\infty}^{\infty} \psi^{\prime}{ }_{a, b}(t) s(t) d t
$$

Now, the original signal $s(t)$ is provided by the inverse transformation shown in Eq. 4-6.

$$
s(t)=\frac{1}{c_{\psi}} \int_{-\infty}^{\infty} \int_{-\infty}^{\infty} S(b, \mathrm{a}) \psi_{a, b}(t) \frac{d a d b}{a^{2}}
$$

MATLAB code has many choices of mother wavelet functions, such as Meyer wavelet, and Morlet wavelet [43]. Selecting suitable mother wavelet function is required to obtain reasonable results. The Continuous Wavelet Transform (CWT) in MATLAB code was used for the post analyses [43]. Different wavelet mother functions were used 
for the comparisons of frequency distributions in the air-ingress phenomenon and the OECD problem. Figure 12 shows examples of two different wavelet mother functions, the Meyer wavelet function and the Morlet wavelet function. The Morlet wavelet function using in MATLAB code is given in Eq. 4-7

$$
\psi(x)=\mathrm{e}^{-x^{2} / 2} \cos (5 x)
$$

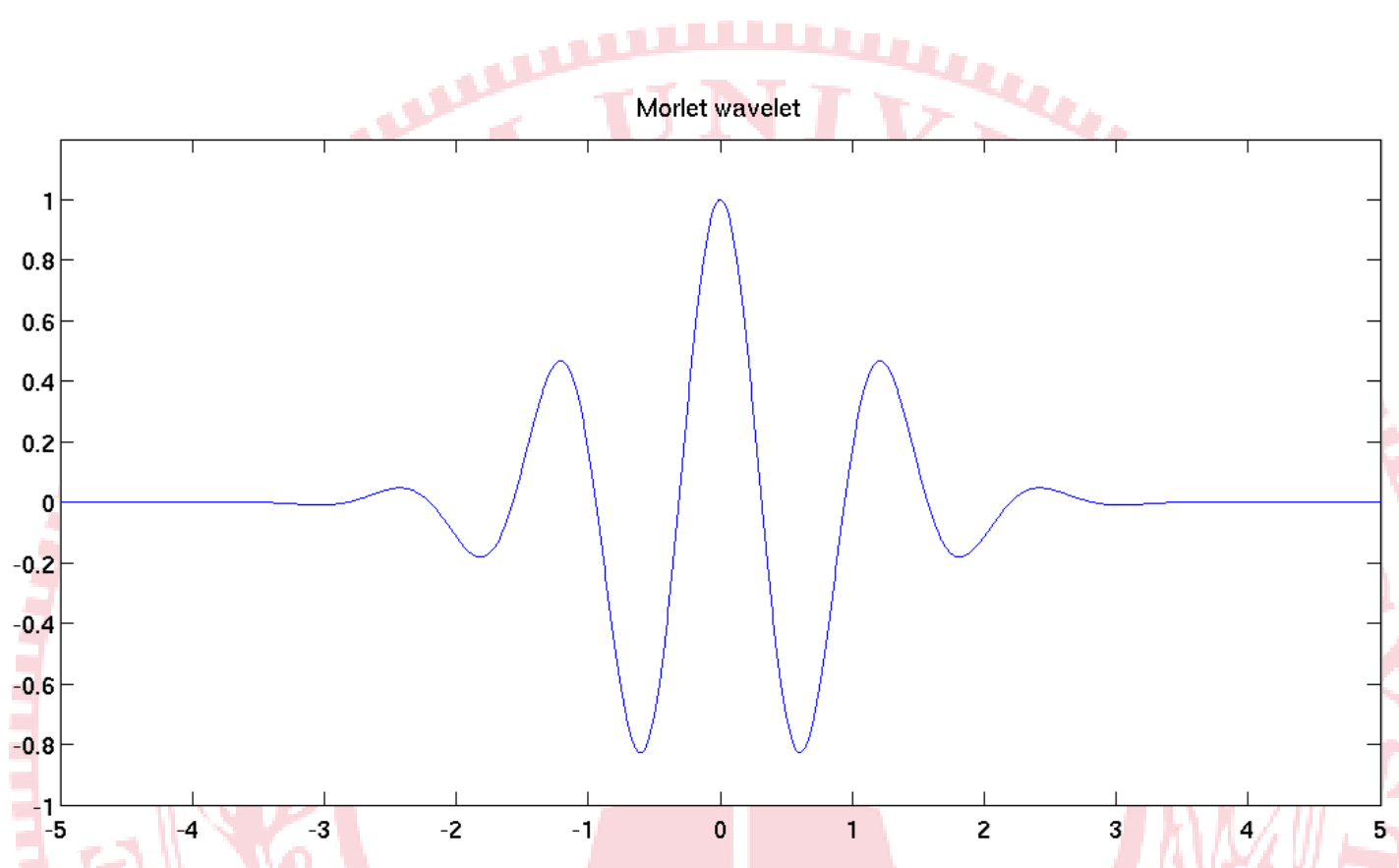

Figure 12. Examples of wavelet mother functions from 5 to -5 with 2048 points: (a) the Meyer wavelet functions, (b) the Morlet wavelet functions.

\subsubsection{Proper orthogonal decomposition}

The flow visualization technique is one of the primary techniques used to identify vortices within experimental flow fields. Particle image velocity (PIV) technique is used to obtain two- and three-dimensional flow fields, and DNS and LES method are utilized for computations of 3D flow fields. The POD method is introduced to help study turbulent velocity fields in the research [46]. The concept of the POD method is to decompose the original signal into many modes with its eigenvectors and eigenvalues which are descended to find the principle directions of the datasets. New signals are reconstructed by selecting the numbers of the modes from the most important to the minimum required by the user. When the least eigenvalues are ignored, the reconstructed fields present the results with large flow patterns that are similar to those results resolved in LES approaches $[45,46]$. Since this method can filter out small 
components and retain the relatively important components, POD helps study flow features and flow patterns [47].

For example, a two-dimensional (2D) dataset is given, and the velocity is expanded as Eq. 4-8.

$$
u_{i}(\mathrm{x}, \mathrm{t})=\sum_{k} \sum_{n} a^{(k, n)}(t) \psi_{i}^{(k, n)}(x)
$$

where $a$ is the time-dependent coefficient corresponding to the orthogonal basis, $\psi_{i}$, for the $i$ th component and is calculated from Eq. 4-9.

$$
a^{(k, n)}(\mathrm{t})=\frac{1}{L_{x_{1}} L_{x_{2}}} \iint u_{i}(\mathrm{x}, \mathrm{t}) \psi_{i}^{*}{ }^{(k, n)}(x) d x
$$

where $L_{x_{1}}$ and $L_{x_{2}}$ are the extents of the domain in $x_{1}$ and $x_{2}$ directions, respectively. The ${ }^{*}$ ) denotes the complex conjugate value. Assuming $\psi_{i}$ is a product of a Fourier basis in $x_{1}$ and an inhomogeneous basis $\left(\phi_{i}\right)$ in $x_{2}$ given by Eq. 4-10.

$$
\psi_{i}^{(k, n)}(x)=\phi_{i}{ }^{(k, n)}\left(x_{2}\right) e^{\frac{i 2 \pi k x_{1}}{L_{x_{1}}}}
$$

where $i=\sqrt{-1}$ inside the exponential term. After doing this, a 2D problem is converted into a 1D POD problem. Here, a POD filter can be defined as a large-scale field and a small-scale field (Eq. 4-11).

$$
u=\bar{u}+u^{\prime \prime}
$$

where the filtered field is shown in Eq. 4-12.

$$
\bar{u}_{i}\left(\mathrm{x}_{1}, \mathrm{x}_{2}\right)=\sum_{k=0}^{K} \sum_{n=0}^{N} H^{(k, n)} a^{(k, n)} \psi_{i}^{(k, n)}\left(x_{1}, x_{2}\right)
$$

In Eq. $4-12, H^{(k, n)}$ is a filter characteristic function to amplify or attenuate the eigenvalues of each POD mode. If no amplification or attenuation is required, $H^{(k, n)}$ is simply 1.

Here, the filtered field can be expressed as Eq. 4-13.

$$
\bar{u}_{i}\left(\mathrm{x}_{1}, \mathrm{x}_{2}\right)=\frac{1}{L_{x_{1} L_{2}}} \iint u_{j}\left(x_{1}^{\prime}, x_{2}^{\prime}\right) h_{i j}\left(x_{1}, x_{2}, x_{1}^{\prime}, x_{2}^{\prime}\right) d x_{1}^{\prime} d x_{2}^{\prime}
$$

where the kernel, $h_{i j}$, is given by Eq. 4-14.

$$
h_{i j}=\sum_{k=0}^{K} \sum_{n=0}^{N} H^{(k, n)} \psi_{i}^{(k, n)}\left(x_{1}, x_{2}\right) \psi_{j}{ }^{(k, n) *}\left(x_{1}, x_{2}\right)
$$

POD method is used to study the air-ingress phenomenon and the rod bundle problem. A MATLAB script was developed to use POD method.

\subsection{Error and Uncertainty Analyses}

The results of CFD predictions are sensitive to many factors, such as mesh sizes and iterative convergence issues. Especially, the mesh size affects significantly the final results of CFD efforts [51]. Verification and validation that are two concerns for quantifying simulation results provide accuracy with high confidence $[52,53]$. Verification relies on solving correct governing equations and selecting proper assumptions and boundary conditions. Instead, a comparison using experimental data to 
assess computations is required for validation [51]. The final solutions of CFD simulations are calculated by using DNS which requires extremely large computations. In order to reduce computational resource, the Richardson extrapolation was used. The solution verification using Richardson Extrapolation and GCI provides error estimations of numerical uncertainty with a numerical error interval. Simulations with higher order of numerical scheme provide higher order of accuracy and less numerical errors. Due to Taylor-series expansions, low order numerical methods seem to have more numerical uncertainty than high order methods. The study of the mesh grid size independency was delivered by using Richardson Extrapolation and grid convergence index (GCI) method [52-55].

\subsubsection{Richardson extrapolation}

Richardson extrapolation is powerful to improve numerical convergence and to serve two practical purposes. First, the extrapolation results can be used to estimate the error due to mesh sizes. The other benefit is using the simulation results of different mesh sizes to extrapolate the actual values [51]. However, the estimated final values are enormously affected by the incomplete iterative convergence errors. When a simulation does not reach convergence, the simulation results change significantly [51]. Using Taylor's expansions which are the most common methods can simply estimate answers with higher order of accuracy [54]. Richardson extrapolation was implemented to quantify the simulation results of the air ingress problem and the OECD rod bundle exercise $[51,54]$.

\subsubsection{Grid convergence index method}

Grid convergence index (GCI) is a study of approaching to quantification of uncertainty in CFD. Similar to any numerical methods, the higher order calculations of GCI have less uncertainty than the lower order calculations [55]. Applying GCI to the results of fine meshes is normally used to obtain the uncertainty with high confidence, but using coarse grids gives at least some certain level confidence of the uncertainty [55]. Besides applying to structure mesh grids, GCI has been developed for the use of

unstructured mesh grids; therefore, GCI has become more common for the quantification of uncertainty in CFD publications [51]. Generally speaking, the second order accuracy is embedded in GUI working usually with Richardson Extrapolation to obtain whole information that contains the estimating value and the corresponding uncertainty [51].

\subsubsection{Example calculations of Richardson extrapolation}

At least three simulation sets with different mesh sizes are prepared to determine values of key variables for applying to Richardson Extrapolation and GCI. The 
approximate relative error, the estimated relative error of the extrapolations, and the uncertainty estimation are requirements for calculating GCI and the corresponding uncertainty. Calculating a representative grid size of structured grids and unstructured grids that are both available for GCI is simply following the calculations as given in Eq. 4-15 and Eq. 4-16, respectively.

$$
\begin{aligned}
h & =\left[\left(\Delta X_{\max }\right)\left(\Delta Y_{\max }\right)\left(\Delta Z_{\max }\right)\right]^{1 / 3} \\
h & =\left[\left(\sum_{i=1}^{N} \Delta V_{i}\right) / N\right]^{1 / 3}
\end{aligned}
$$

The grid refinement factors, $r$, that are defined as the ratio of two representative grid sizes are suggested to be greater than 1.3. The constant and non-constant $r$ can both be applied to calculate the observed order (p), which is used for Richardson extrapolation and GCI. The calculations of the observed order (p) with constant or nonconstant $r$ are given by Eq. 4-17 and Eq. 4-18, respectively.

$$
\begin{aligned}
& p_{\text {const }}=\frac{|\ln | \varepsilon_{32} / \varepsilon_{21}||}{\ln \left(r_{21}\right)} \\
& p_{\text {non-const }}=\frac{|\ln | \varepsilon_{32} / \varepsilon_{21}|+q(p)|}{\ln \left(r_{21}\right)}
\end{aligned}
$$

where $\varepsilon_{32}$ and $\varepsilon_{21}$ are absolute difference between two simulation results. $r_{21}$ is the grid refinement factor of the second dataset to the first dataset. $q(p)$ is defined in Eq. 4-19.

$$
\begin{aligned}
& q(p)=\ln \left(\frac{r_{21}^{p}-s}{r_{32}^{p}-s}\right) \\
& s \equiv 1 * \operatorname{sign}\left(\varepsilon_{32} / \varepsilon_{21}\right)
\end{aligned}
$$

The observed order is used to calculate the extrapolated values of the unknown variable given by Eq. 4-21.

$$
\phi_{\text {ext }}^{21}=\left(r_{21}^{p} \phi_{1}-\phi_{2}\right) /\left(r_{21}^{p}-1\right)
$$

Finally, the observed order, the approximate relative error, and the estimated relative error of the extrapolations are utilized for GCI that is further applied for calculating uncertainty (Eq. 4-22).

$$
u_{\text {numerical }}=G C I / k
$$

where $\mathrm{k}$ defined as the error distribution is 1.15 for a shifted Gaussian and is 2 for a Gaussian distribution. 


\section{CHAPTER V}

\section{RESULTS AND ANALYSIS}

The study of air ingress phenomena contained five sub-topics: the mesh size sensitivity study, two fluid pairs (gas-gas and liquid-liquid), two geometries (short and long pipe), the moving valve conditions (fully open and moving), and two turbulent models (RANS and LES). Table 3 lists the results of eight scenarios that were simulated and two experiments in the air ingress study. From Case 1-3, the mesh size sensitivity study was given. Case 3 and 4 were used for the valve moving comparison. The turbulent modeling comparison was simulated in Case 4 and 5 . The effects of the pipe lengths were done in Case 5 and 6 and Case 7 and 8 with $\mathrm{He}_{-} \mathrm{SF}_{6}$ and water-brine, respectively. Case 7 and 8 were the simulations representing the experiments. Case 9 and 10 were the experiments listed in Table 3.

The comparisons of scenarios with different sub-topics were listed in Table 4 where, for example, the mesh size sensitivity study was listed as the first category. Two subsequent sub-sections discuss a liquid ingress phenomenon (Case 7-10) and a gas ingress phenomenon (Case 1-6), respectively.

Table 3. Lists of inner and outer maximum wave front velocity and the corresponding ratios of simulations in the air ingress problem.

\begin{tabular}{|c|c|c|c|c|c|c|c|c|}
\hline Case & Mesh Size & Pipe & Valve & Turbulence & Inner Vel. & Outer Vel. & I/O Ratio & Fluid Pair \\
\hline $1 \xi$ & $1.5 \mathrm{~mm}$ & Short & Fully Open & Realizable k- $\varepsilon$ & $0.955 \mathrm{~m} / \mathrm{s}$ & $1.112 \mathrm{~m} / \mathrm{s}$ & $85.9 \%$ & He-SF6 \\
\hline 2 & $1.0 \mathrm{~mm}$ & Short & Fully Open & Realizable k- $\varepsilon$ & $0.944 \mathrm{~m} / \mathrm{s}$ & $1.104 \mathrm{~m} / \mathrm{s}$ & $85.5 \%$ & $\mathrm{He}-\mathrm{SF} 6$ \\
\hline 3 & $0.7 \mathrm{~mm}$ & Short & Fully Open & Realizable k- $\varepsilon$ & $0.924 \mathrm{~m} / \mathrm{s}$ & $1.099 \mathrm{~m} / \mathrm{s}$ & $84.1 \%$ & He-SF6 \\
\hline 4 & $0.7 \mathrm{~mm}$ & Short & Moving & Realizable k- $\varepsilon$ & $0.699 \mathrm{~m} / \mathrm{s}$ & $0.900 \mathrm{~m} / \mathrm{s}$ & $77.7 \%$ & He-SF6 \\
\hline 5 & $0.7 \mathrm{~mm}$ & Short & Moving & LES & $0.723 \mathrm{~m} / \mathrm{s}$ & $0.905 \mathrm{~m} / \mathrm{s}$ & $79.9 \%$ & He-SF6 \\
\hline 6 & $0.7 \mathrm{~mm}$ & Long & Moving & LES & $0.881 \mathrm{~m} / \mathrm{s}$ & $1.071 \mathrm{~m} / \mathrm{s}$ & $82.3 \%$ & He-SF6 \\
\hline 7 & $0.7 \mathrm{~mm}$ & Short & Moving & LES & $0.134 \mathrm{~m} / \mathrm{s}$ & $0.168 \mathrm{~m} / \mathrm{s}$ & $79.8 \%$ & Water-Brine \\
\hline 8 & $0.7 \mathrm{~mm}$ & Long & Moving & LES & $0.142 \mathrm{~m} / \mathrm{s}$ & $0.172 \mathrm{~m} / \mathrm{s}$ & $82.6 \%$ & Water-Brine \\
\hline 9 & Experiment & Short & Moving & $\mathrm{X}$ & $\mathrm{X}$ & $0.164 \mathrm{~m} / \mathrm{s}$ & & Water-Brine \\
\hline 10 & Experiment & Long & Moving & $X$ & $X$ & $0.168 \mathrm{~m} / \mathrm{s}$ & & Water-Brine \\
\hline 11 & Theory & $\mathrm{X}$ & $\mathrm{X}$ & $\mathrm{X}$ & & $0.1604 \mathrm{~m} / \mathrm{s}$ & & Water-Brine \\
\hline
\end{tabular}


Table 4. Comparisons between different cases.

\begin{tabular}{lllll}
\hline Case Comparison & Inner Difference & Inner Ratio & Outer Difference & Outer Ratio \\
\hline $1,2, \& 3$ (Mesh) & $0.031 \mathrm{~m} / \mathrm{s}$ & $3.25 \%$ & $0.013 \mathrm{~m} / \mathrm{s}$ & $1.17 \%$ \\
$3 \& 4$ (Valve) & $0.225 \mathrm{~m} / \mathrm{s}$ & $24.35 \%$ & $0.199 \mathrm{~m} / \mathrm{s}$ & $18.11 \%$ \\
$4 \& 5$ (Turbulent) & $0.024 \mathrm{~m} / \mathrm{s}$ & $3.32 \%$ & $0.005 \mathrm{~m} / \mathrm{s}$ & $0.55 \%$ \\
$5 \& 6\left(\right.$ Pipe $\left.^{1}\right)$ & $0.158 \mathrm{~m} / \mathrm{s}$ & $17.93 \%$ & $0.166 \mathrm{~m} / \mathrm{s}$ & $15.50 \%$ \\
$7 \& 8\left(\right.$ Pipe $\left.^{2}\right)$ & $0.008 \mathrm{~m} / \mathrm{s}$ & $5.63 \%$ & $0.004 \mathrm{~m} / \mathrm{s}$ & $2.33 \%$ \\
$9 \& 10\left(\right.$ Pipe $\left.^{3}\right)$ & $\mathrm{X}$ & $\mathrm{X}$ & $0.004 \mathrm{~m} / \mathrm{s}$ & $2.38 \%$ \\
$7 \& 9(*)$ & $\mathrm{X}$ & $\mathrm{X}$ & $0.004 \mathrm{~m} / \mathrm{s}$ & $2.38 \%$ \\
$8 \& 10\left(^{*}\right)$ & $\mathrm{X}$ & $\mathrm{X}$ & $0.004 \mathrm{~m} / \mathrm{s}$ & $2.33 \%$ \\
\hline
\end{tabular}

1: With $\mathrm{He}_{-} \mathrm{SF}_{6}$ fluid pairs

2: With water-brine fluid pairs

3: Comparison for the experiments

*. Comparison for the experiments and simulations

\subsection{Grid Sensitivity Study}

Numerical diffusion occurs in discretizing differential equations, such as the Navier-Stokes equations. To achieve high simulation fidelity, the mesh size sensitivity study is delivered to minimize the numerical diffusion and to determine the appropriate mesh size. The Richardson extrapolation is introduced to accomplish the goal of the grid sensitivity study and the corresponding uncertainty.

Starting with the mesh size sensitivity study, three simulations with the mesh sizes of $1.5 \mathrm{~mm}, 1.0 \mathrm{~mm}$, and $0.7 \mathrm{~mm}$ were listed as Case 1, Case 2, and Case 3 in Table 3 . The fully open valve condition with unsteady Realizable k- $\varepsilon$ model, the short pipe geometry, and He-SF6 fluid pairs were used for the grid sensitivity study [50]. The extrapolated results and corresponding relative errors were calculated to confirm that the reliable results were obtained. The results of the three mesh sizes are shown in Figure 13. Table 3 shows that the relative difference is $3.25 \%$ and $1.17 \%$ for the inner pipe and the outer pipe, respectively. Figure 14 and Figure 15 show the velocity magnitudes and relative errors for the extrapolations and the simulations with different mesh sizes. The definitions of calculated relative errors were seen in Eq. 5-1.

$$
e_{\text {ext }}^{21}=\left|\frac{\phi_{\text {ext }}^{21}-\phi_{1}}{\phi_{\text {ext }}^{21}}\right|
$$

It was obvious that three simulation results gave the relative errors within $4 \%$ (Figure 14). The $1.5 \mathrm{~mm}$ mesh size gave better predictions with the Richardson Extrapolation than the $1.0 \mathrm{~mm}$ simulation which predicted large discrepancy with the 
other simulations and the extrapolation. The results with $0.7 \mathrm{~mm}$ mesh size presented the best agreement with the Richardson Extrapolation within 1\% of relative errors. Although the results of $1.5 \mathrm{~mm}$ and $1.0 \mathrm{~mm}$ showed similar calculations and had the relative errors within $4 \%$, the $0.7 \mathrm{~mm}$ mesh size showed the best predictions in three simulations. The $0.7 \mathrm{~mm}$ mesh size was chosen for the further simulations in this air ingress problem.

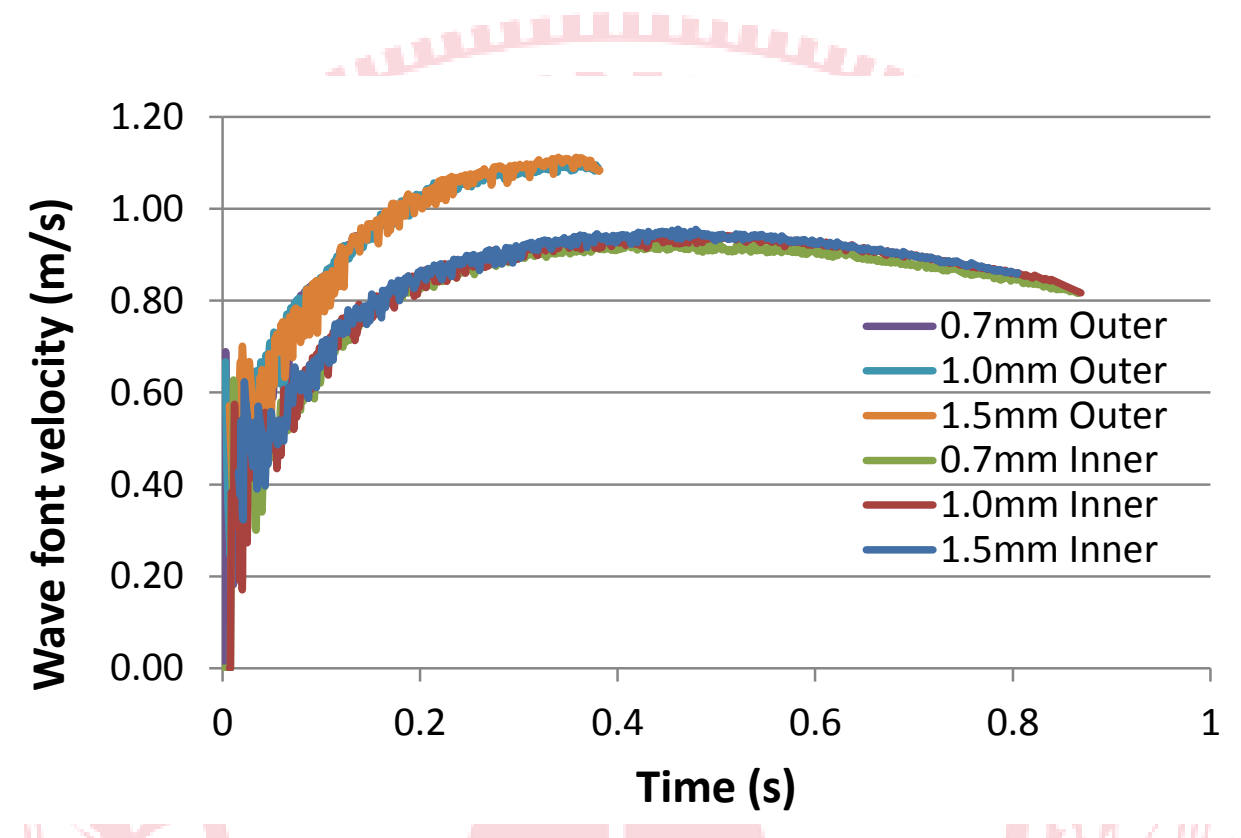

Figure 13. Comparison of wave front velocity profile of the mesh sensitivity study. 


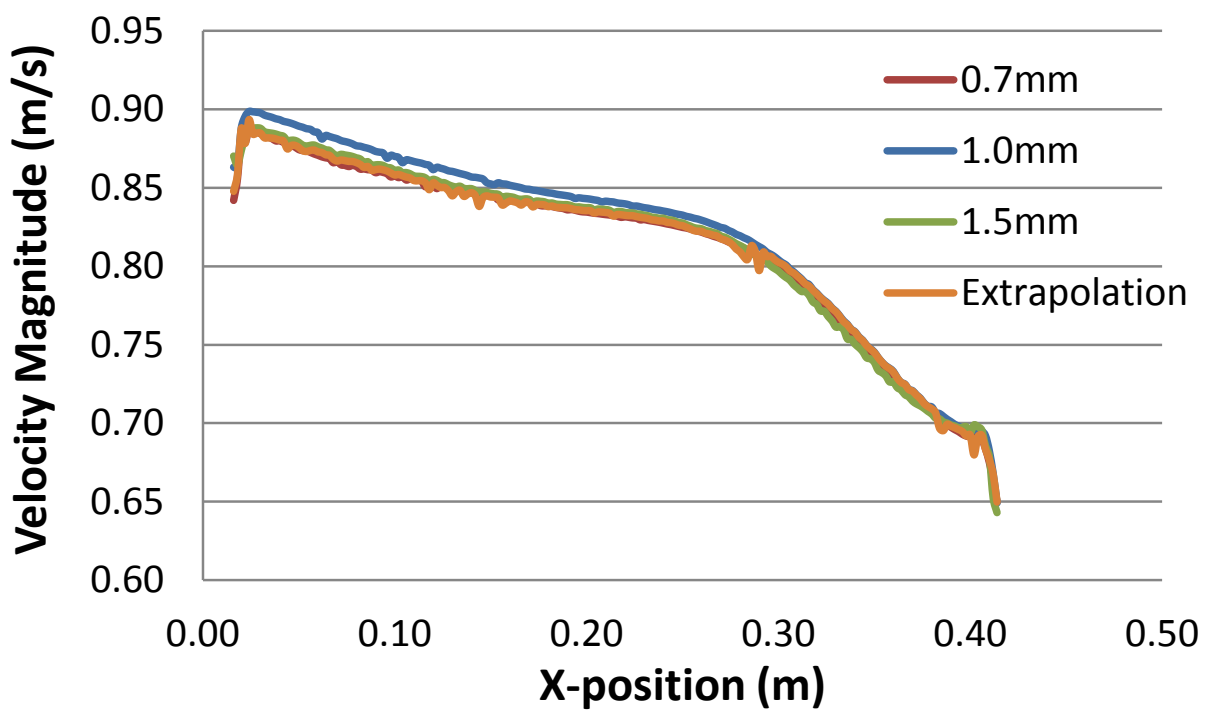

Figure 14. Velocity magnitudes of the fluid along with $x$-axis for 200 locations in the simulations and the Richardson Extrapolation.

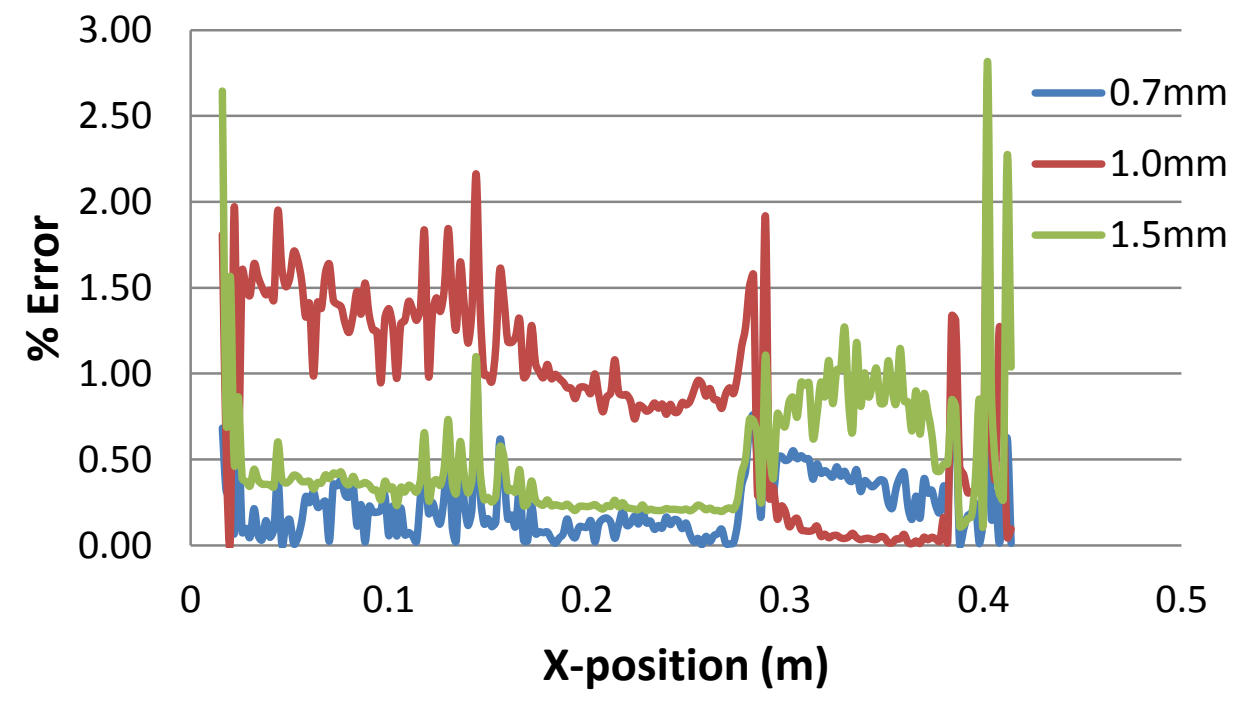

Figure 15. Relative errors comparing with the extrapolations along with $x$-axis for 200 locations in three mesh sizes. 


\subsection{Water Ingress Phenomenon}

Two water-brine cases using different pipe length were simulated as Case 7 and 8 listed in Table 3 . The finest mesh size $(0.7 \mathrm{~mm})$, the LES turbulent model, and two geometries (long and short pipes) were used in the water-brine simulations. The evolutions of the wave front velocity in the co-axial pipes and in the lower plenum are shown in Figure 16 and Figure 17, respectively. The maximum wave front velocities were defined as the maximum values during the velocity evolution of the pipe. The maximum inner velocities were $0.134 \mathrm{~m} / \mathrm{s}$ and $0.142 \mathrm{~m} / \mathrm{s}$ for the short and the long pipes, respectively.

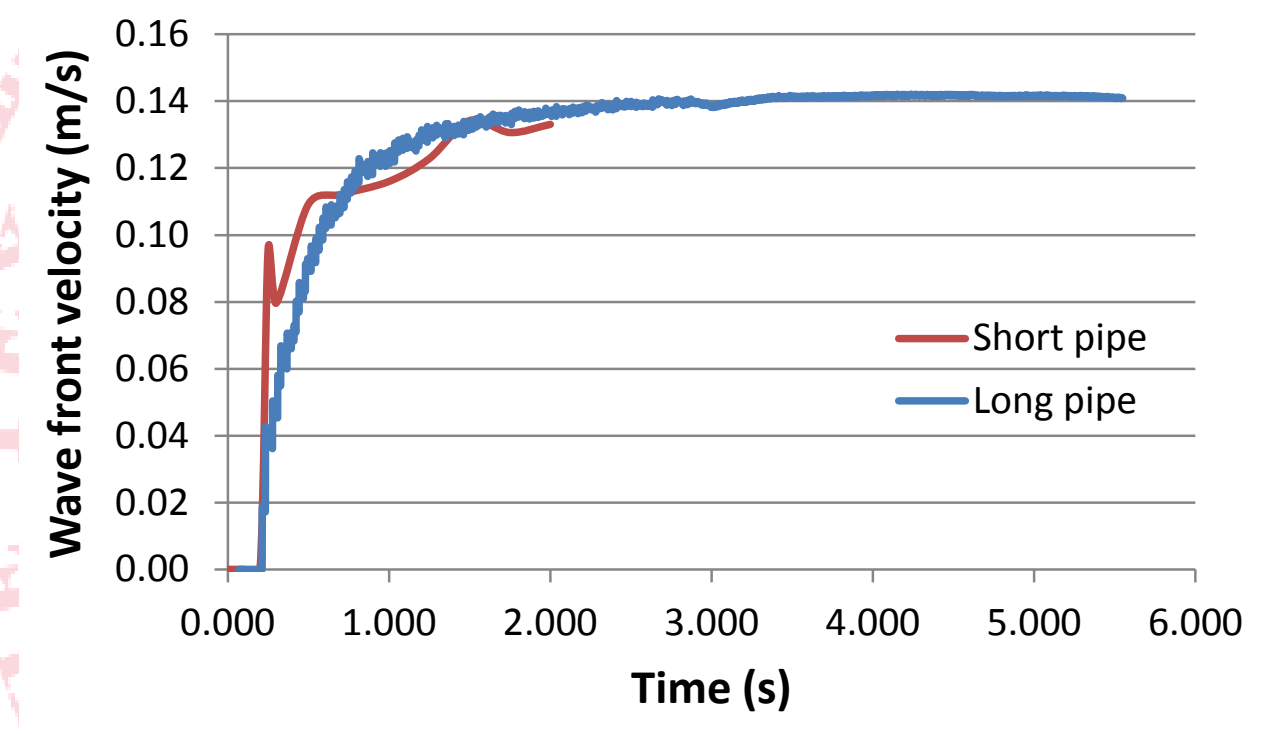

Figure 16. The evolutions of the wave front velocity in the inner pipe for the simulations with different pipe lengths. 


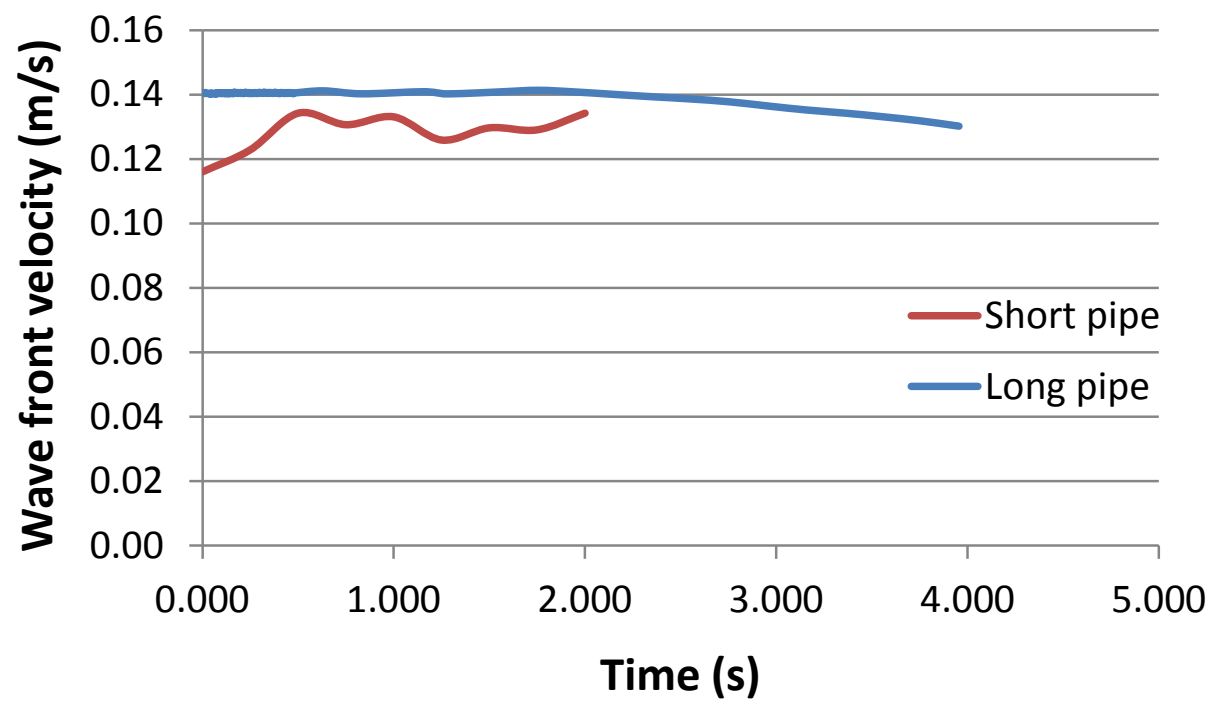

Figure 17. The evolutions of the spreading rate in the lower plenum for the simulations with different pipe lengths.

In these simulations, the maximum wave front velocities were considered as the terminal velocities. The maximum wave front velocities of the outer pipe were $0.168 \mathrm{~m} / \mathrm{s}$ and $0.172 \mathrm{~m} / \mathrm{s}$ for the short and the long geometries, respectively. The absolute difference was simply calculated as $0.008 \mathrm{~m} / \mathrm{s}$ and $0.004 \mathrm{~m} / \mathrm{s}$ for inner and outer pipes by using Eq. 5-2. The definitions of calculated relative difference were given in Eq. 5-3. According to Benjamin's theorem, the wave front velocity is related to the density ratio and the height of the fluid (or the height of the pipe) [5]. Thus, the theoretical terminal velocity is calculated as $0.1604 \mathrm{~m} / \mathrm{s}$ for the outer pipe. The simulation results gave the relative error of $4.74 \%$ for the short pipe case where the experimental data showed the relative error of $2.24 \%$.

$$
\begin{aligned}
& \left\{\begin{array}{l}
\Delta V_{\text {inner }}=V_{\text {inner }}(\text { long })-V_{\text {inner }}(\text { short }) \\
\Delta V_{\text {outer }}=V_{\text {outer }}(\text { long })-V_{\text {outer }}(\text { short })
\end{array}\right. \\
& \left\{\begin{array}{l}
\frac{\Delta V_{\text {inner }}}{V_{\text {inner }}(\text { long })}=100 \% \frac{V_{\text {inner }}(\text { long })-V_{\text {inner }}(\text { short })}{V_{\text {inner }}(\text { long })} \\
\frac{\Delta V_{\text {outer }}}{V_{\text {outer }}(\text { long })}=100 \% \frac{V_{\text {outer }}(\text { long })-V_{\text {outer }}(\text { short })}{V_{\text {outer }}(\text { long })}
\end{array}\right.
\end{aligned}
$$

The pipe lengths from the valve to the light fluid tank were used to simulate the distance from the break locations to the reactor vessel. After simulations, the geometry effects from changing a short pipe to a long pipe showed a slight enhancement of the 
maximum wave front velocity. Theoretically, the maximum velocity is affected by the diameter of the pipe and the density ratio of the fluid pair, so it is regarded to have a constant maximum velocity. The maximum wave front velocities of the inner and the outer pipes increased with the magnitudes of $5.63 \%$ and $2.33 \%$, respectively. According to Eq. 5-4, the ratio of the maximum velocity of the inner pipe to the outer pipe for the short and the long geometries were $79.8 \%$ and $82.6 \%$, respectively. Comparing with the theoretical value of $0.1604 \mathrm{~m} / \mathrm{s}$. the simulation result and the experimental data provided $7.23 \%$ and $4.74 \%$, respectively.

$$
\text { Velocity Ratio }=100 \% \frac{V_{\text {inner }}}{V_{\text {outer }}}
$$

Based on the experimental data, the wave front velocities of the outer pipe were $0.164 \mathrm{~m} / \mathrm{s}$ and $0.168 \mathrm{~m} / \mathrm{s}$ for the short and the long geometries (Table 3 ). The wave front velocity of the inner pipe was difficult to be analyzed because it was challenging to distinguish the inner wave fronts. Figure 18 shows that the inner wave front is difficult to be measured.
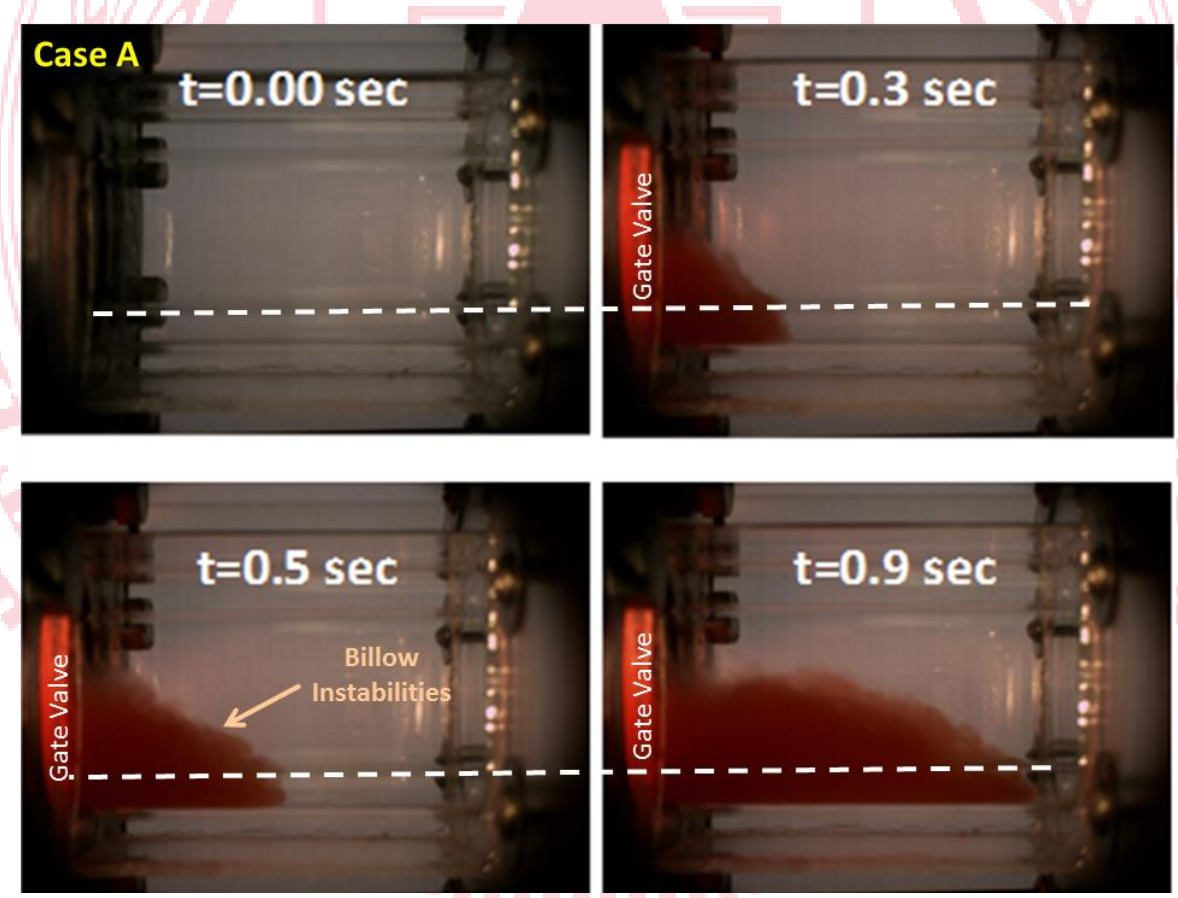

Figure 18. Examples of experimental snapshots for the gravity-current propagation of the water-brine scenario with the short pipe geometry [39]. 
Depending on the experimental data, the relative difference of the outer wave front velocities between the short and long pipes is $2.38 \%$ (Eq. 5-3). In other words, the maximum wave front velocities in the experiments increased with the magnitudes of $2.38 \%$ which was approximately the same as the value obtained by the simulations. These results were summarized in Table 3 and Table 4.

The wave front behaviors of the outer pipe were shown in Figure 19 and Figure 20. In both short pipe and long pipe comparisons, the simulations predicted lower values for the wave front locations and velocities than the experimental data. The initial rapid burst was observed in both short pipe and long pipe cases of the experiments and the simulations. Even though the experimental data showed more noticeable initial burst than those of the simulations, the terminal velocities of the wave fronts between experiments and simulations did not show significant difference. Figure 19 (b) shows that the wave front velocity from the experiments suddenly jumps to a peak of $0.242 \mathrm{~m} / \mathrm{s}$ after the valve was removed. The wave front velocity, then, rapidly decreased to the terminal velocity of $0.164 \mathrm{~m} / \mathrm{s}$ after approximately 0.7 seconds. On the other hand, the wave front velocity of the simulations shows two plateaus in Figure 19 (b). The wave front velocity from the simulations jumped to a value of $0.120 \mathrm{~m} / \mathrm{s}$ which was half of the experimental value and remained approximately constant till the valve fully open in the simulation. After the valve was fully open, the simulation velocity rapidly accelerated to the terminal velocity of $0.168 \mathrm{~m} / \mathrm{s}$ and remained approximately constant. The initial rapid burst appeared in the long pipe scenario of the experiments in Figure 20 (b), and rapidly decreased as the same phenomenon in the short pipe scenario. 


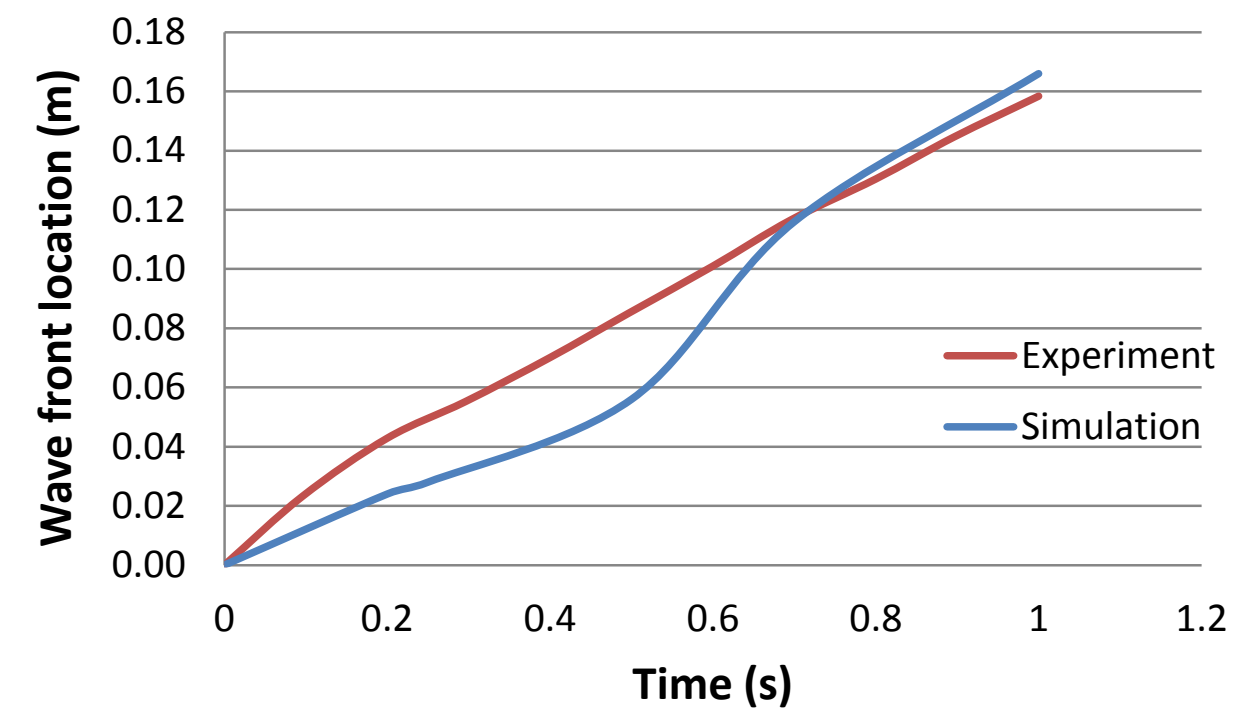

(a)
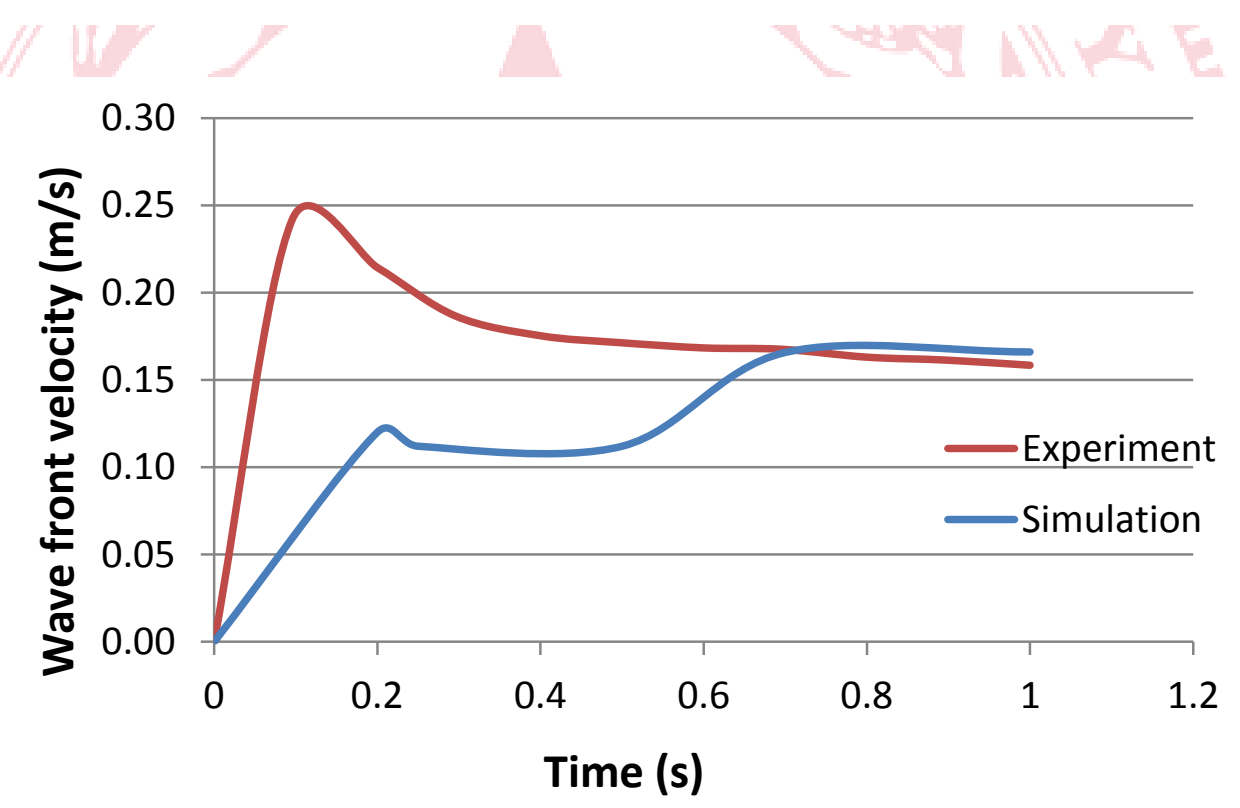

(b)

Figure 19. Results of the outer wave fronts in the short pipe geometry between simulations and experiments. (a) wave front locations; (b) wave front velocities. 


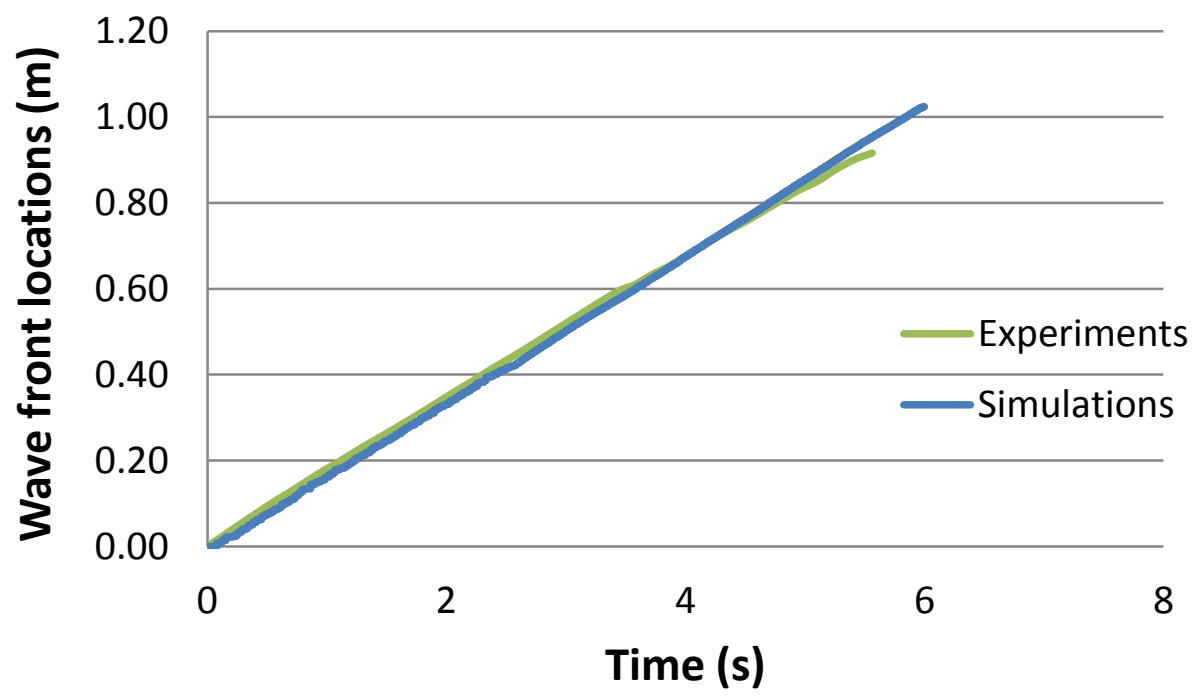

(a)

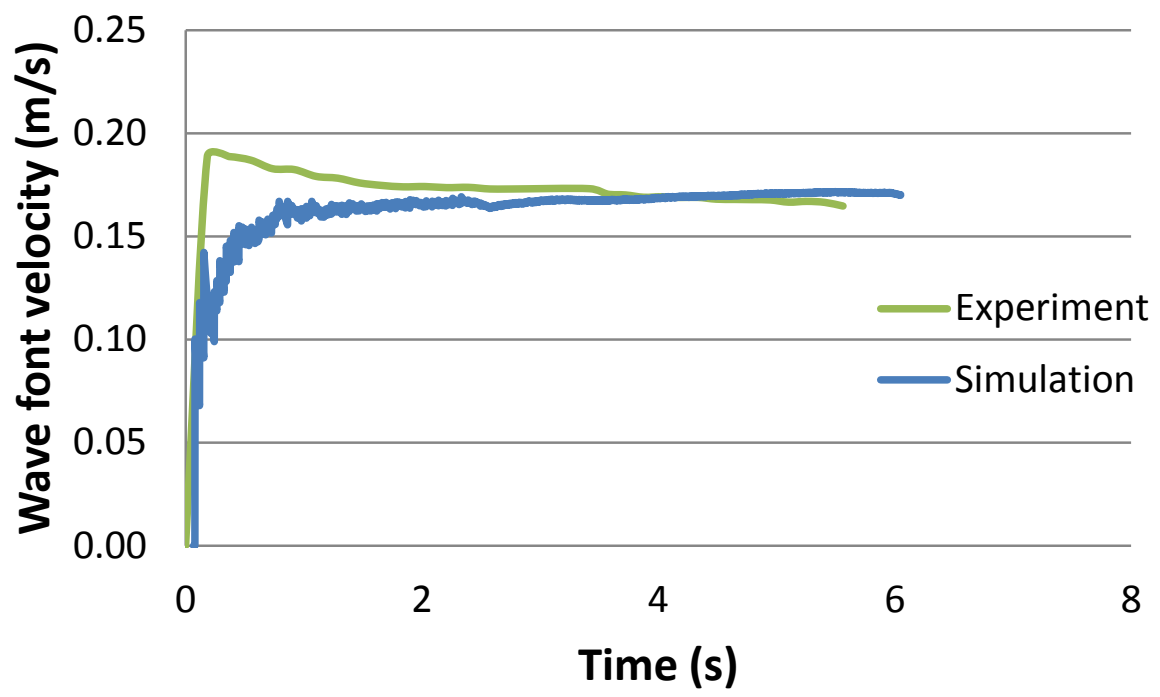

(b)

Figure 20. Results of the outer wave fronts in the long pipe geometry between simulations and experiments. (a) wave front locations; (b) wave front velocities.

It took longer for the heavy fluids to reach terminal velocities for both experiment and simulation in the long pipe scenario. These discrepancies between the experiment and the simulation during the time that the valve was opened were probably caused by the moving valve assumption. It was believed that the valve was moved faster 
at the beginning in the experiments than the simulations where the valve was set to move with a constant speed. Assuming the valve moved with a constant speed was probably an appropriate approximation because the valve information obtained from the experiment was the time that the valve was opened. Further analyses were required to examine this assumption. Eq. 5-5 and Eq. 5-6 were used to calculate the absolute difference and the relative difference between the simulation results and experimental data.

The comparison gave the difference within $2.4 \%$ for the short and the long pipes cases listed in Table 4. Moreover, the wave fronts in the simulation finally reached the same locations and velocities as those in the experiment. This showed that it was appropriate to assume the valve to move with a constant speed, although the discrepancies were shown at the initial state. As a result, this assumption of steadily moving valve was applicable for the gas-gas simulations.

$$
\begin{aligned}
& \Delta V_{\text {outer }}=V_{\text {outer }}(\text { simulation })-V_{\text {outer }}(\text { experiment }) \\
& \frac{\Delta V_{\text {outer }}}{V_{\text {outer }}(\text { simulation })}=100 \% \frac{V_{\text {outer }}(\text { simulation })-V_{\text {outer }}(\text { experiment })}{V_{\text {outer }}(\text { simulation })}
\end{aligned}
$$

Fast Fourier Transform (FFT) was used to analyze the frequencies in the air ingress phenomenon. The line-probes of the simulations were used to plot FFT spectra. The frequency based power spectra of the wave front velocity evolution were analyzed in this section.

Figure 21 and Figure 22 show the frequency power spectra for the cases of the short pipe and the long pipe, respectively. The time step in both cases was one millisecond (1ms). The samples of the short and the long pipes were 512 and 4096, respectively. Thus, the frequencies in FFT were calculated as 1.953 to $500(1 / \mathrm{s})$ for the short pipe case and as 0.244 to $500(1 / \mathrm{s})$ for the long pipe scenario. Both figures show that the significant frequencies of the flow evolutions were approximately less than $4 \mathrm{~Hz}$ for different locations in the liquid-liquid ingress phenomenon.

\subsection{Gas Ingress Phenomenon}

The grid independent study proved that the $0.7 \mathrm{~mm}$ mesh size was enough fine to obtain high fidelity simulation result in Section 6.1.1. The moving valve assumption was proved to be a good agreement with the experimental data in Section 6.1.2.

Consequently, the turbulent models were used to study the difference between the unsteady RANS and the LES approaches as the Case 4 and Case 5 listed in Table 3 whose corresponding comparisons ( $4 \& 5$ ) could be seen in Table 4. Two helium-SF6 simulations, with the same geometry and mesh size $(0.7 \mathrm{~mm})$ but using different turbulence models (Realizable k- $\varepsilon$ and LES) are discussed here. In the simulations, a moving valve was modeled to represent the experiment including a valve opening time of 0.534 seconds. 


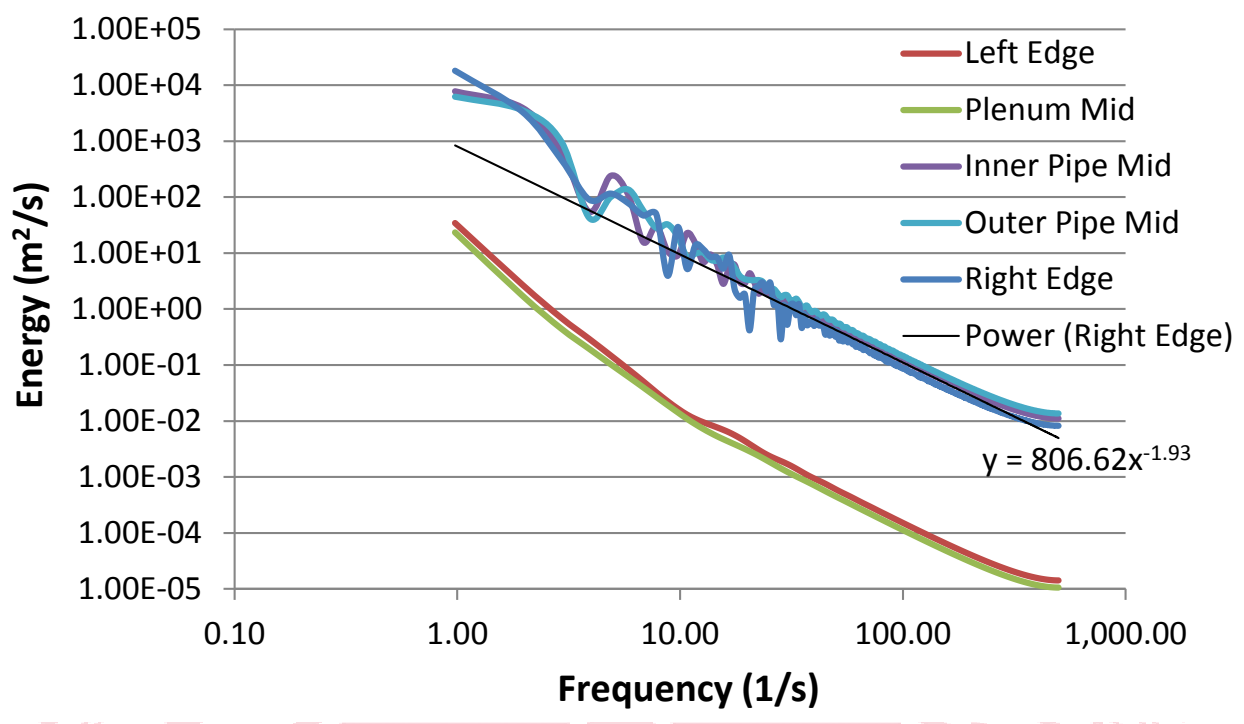

Figure 21. Frequency power spectra of the velocity evolutions at certain locations with water-brine fluids, the LES model, short pipe, and $0.7 \mathrm{~mm}$ mesh size.

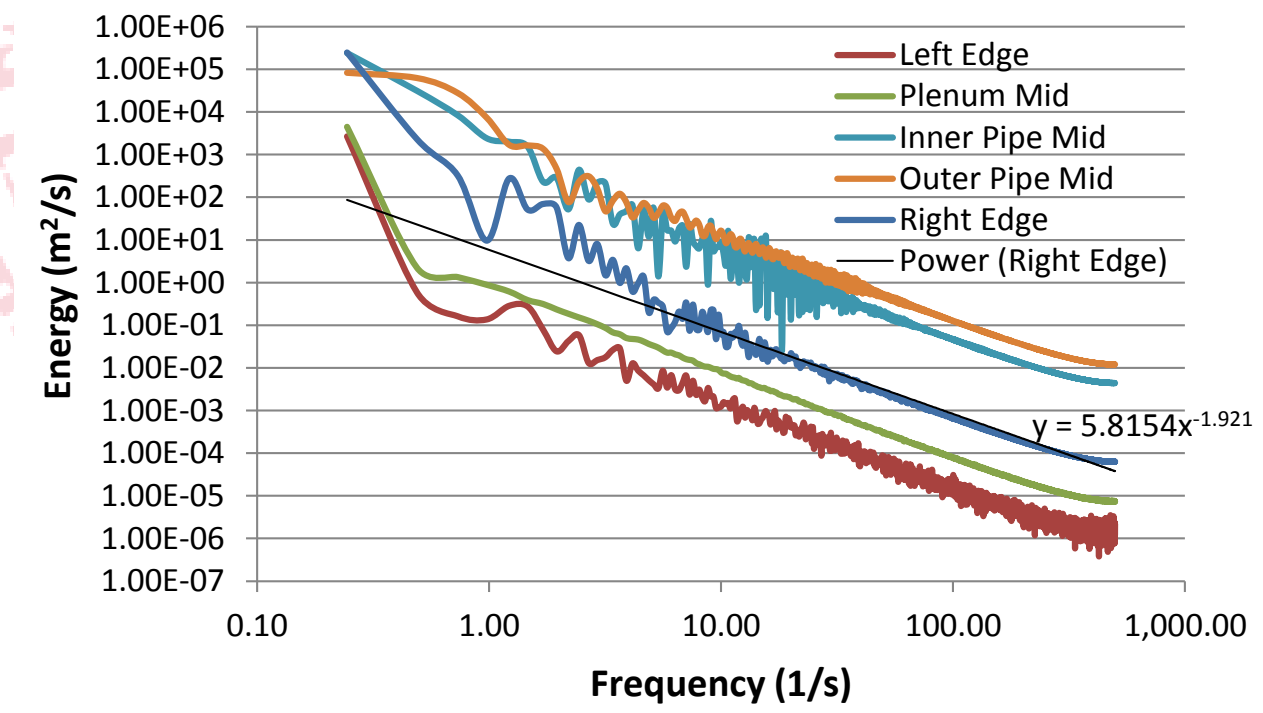

Figure 22. Frequency power spectra of the velocity evolutions at certain locations with water-brine fluids, the LES model, long pipe, and $0.7 \mathrm{~mm}$ mesh size. 
The wave front velocity evolutions of the inner and outer pipes are shown in Figure 23 and Figure 24. The LES result predicted a larger maximum wave front velocity than the unsteady Realizable k- $\varepsilon$ model for the inner and the outer pipes. In the inner pipes, the LES and the Realizable k- $\varepsilon$ models showed the maximum velocities of $0.723 \mathrm{~m} / \mathrm{s}$ and $0.699 \mathrm{~m} / \mathrm{s}$. In the outer pipes, the magnitudes of the maximum wave front velocity from the LES and the Realizable k- $\varepsilon$ predictions were $0.905 \mathrm{~m} / \mathrm{s}$ and $0.900 \mathrm{~m} / \mathrm{s}$, respectively. The values are listed as the Case 4 and Case 5 in Table 3. Through Eq. 5-7, the absolute differences between the two models were $0.024 \mathrm{~m} / \mathrm{s}$ and $0.005 \mathrm{~m} / \mathrm{s}$ for the inner pipe and the outer pipe, respectively. The results obtained through LES simulations provided a slightly different value of $3.32 \%$ from that of the Realizable k- $\varepsilon$ simulations for the inner pipe and a different value of $0.44 \%$ for the outer pipe (Eq. 5-8). The values are listed in Table 2 as the comparison 4-5. This shows that the simulation results have good agreements with the experimental data in the liquid-liquid scenario. Based on the results, the ratio of maximum wave front velocity of the inner pipe to the outer pipe (Eq. $5-4$ ) was $79.9 \%$ for the LES approach, and the value was $77.7 \%$ for the Realizable $\mathrm{k}-\varepsilon$ model.

$$
\begin{aligned}
& \left\{\begin{array}{l}
\Delta V_{\text {inner }}=V_{\text {inner }}(L E S)-V_{\text {inner }}(k-\varepsilon) \\
\Delta V_{\text {outer }}=V_{\text {outer }}(L E S)-V_{\text {outer }}(k-\varepsilon)
\end{array}\right. \\
& \left\{\begin{array}{l}
\frac{\Delta V_{\text {inner }}}{V_{\text {inner }}(k-\varepsilon)}=100 \% \frac{V_{\text {inner }}(L E S)-V_{\text {inner }}(k-\varepsilon)}{V_{\text {inner }}(k-\varepsilon)} \\
\frac{\Delta V_{\text {outer }}}{V_{\text {outer }}(k-\varepsilon)}=100 \% \frac{V_{\text {outer }}(L E S)-V_{\text {outer }}(k-\varepsilon)}{V_{\text {outer }}(k-\varepsilon)}
\end{array}\right.
\end{aligned}
$$




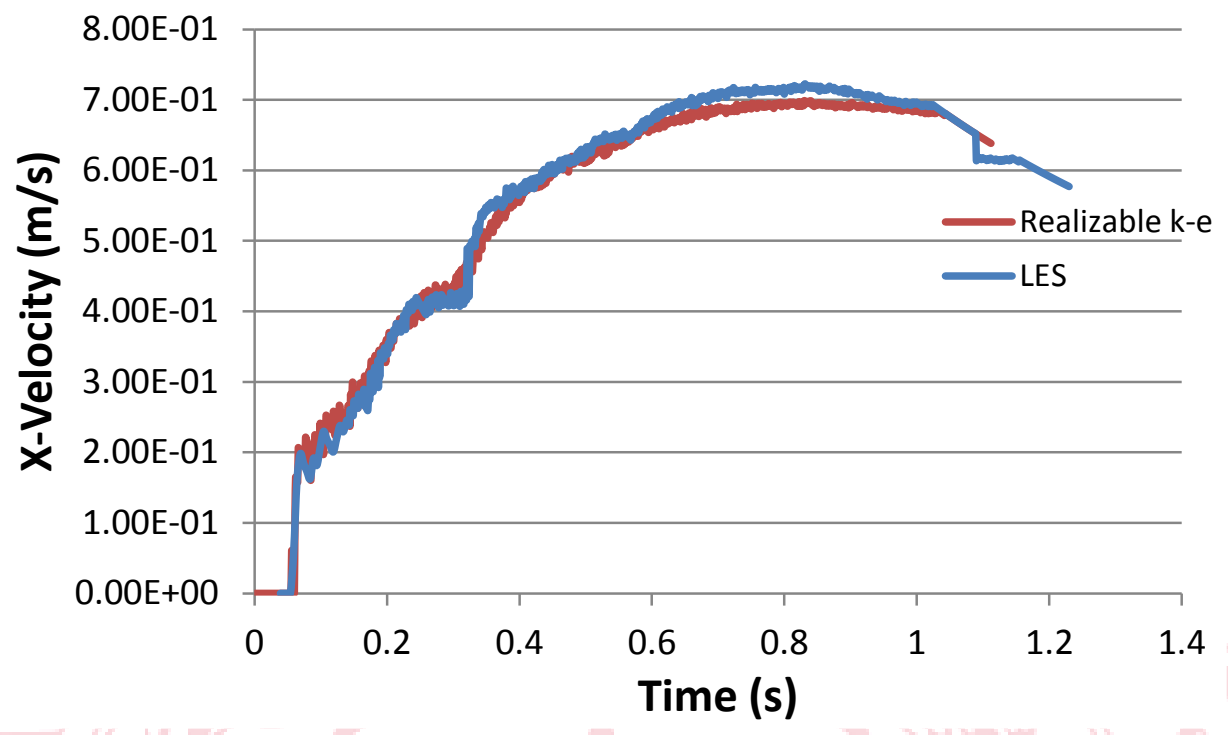

Figure 23. Wave front velocity evolution in the inner pipe for two turbulent models in $0.7 \mathrm{~mm}$ mesh size.

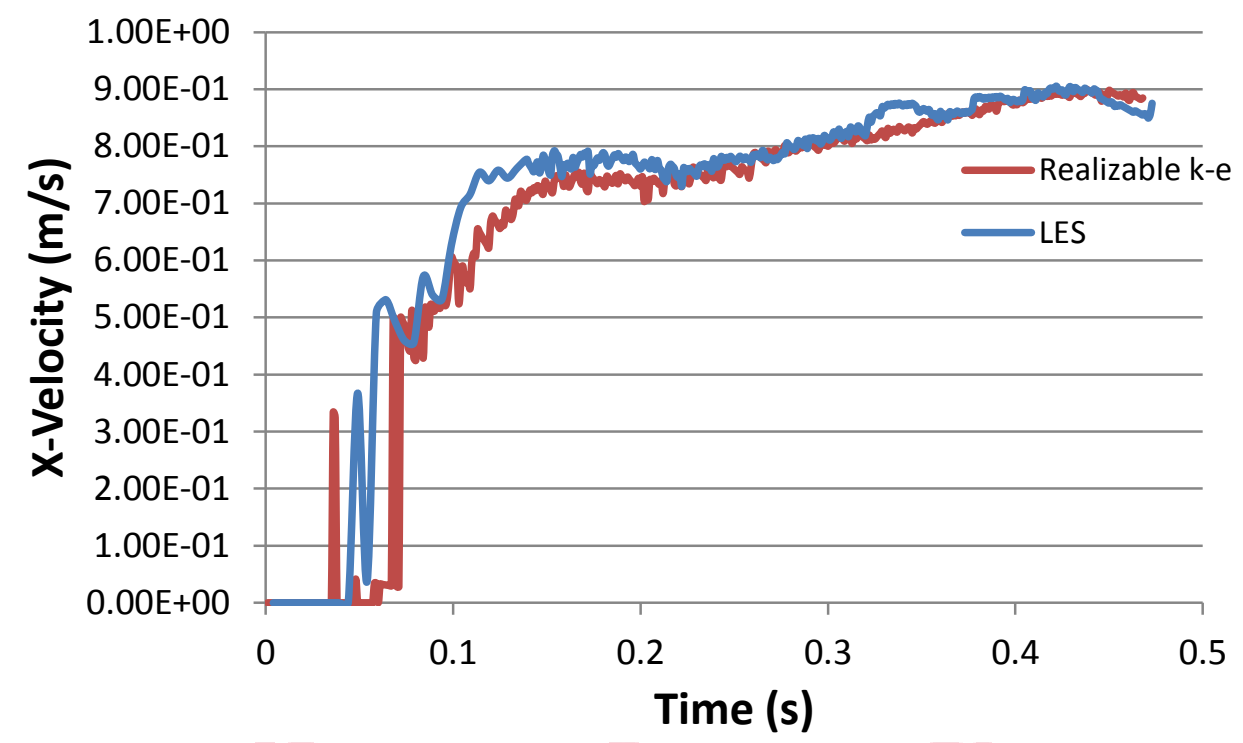

Figure 24. Wave front velocity evolution in the outer pipe for two turbulent models in $0.7 \mathrm{~mm}$ mesh size. 
The FFT analysis was used to create energy spectra to analyze the results of both LES and RANS simulations. For the frequency based spectra, a certain location is fixed, and the time-series velocities are recorded. The magnitudes of the energy were calculated from the results of Fourier transform and its conjugates value. Five locations were selected from the two line probes to compare the energy spectra of LES approaches with those of RANS model. Figure 25 and Figure 26 show the frequency based energy spectra in log-log scale. The FFT results of the Realizable k- $\varepsilon$ model were similar to the LES predictions. The significant frequencies were approximately less than $5 \mathrm{~Hz}$ for both turbulent models. The smallest resolving frequency in the figure depended on the total number of the samples, and the largest resolving frequency was affected by the total samples or time steps. For example, the time step was 1 millisecond with the total number of 512 samples in the LES case; therefore, the frequency range was from 1.9531 $\mathrm{Hz}$ to $500 \mathrm{~Hz}$. In the RANS simulation, the time step was 1 millisecond with a total number of 1024 samples, so the frequency range was from $0.9766 \mathrm{~Hz}$ to $500 \mathrm{~Hz}$.

It should be mentioned that the moving valve is not included in the set of the grid independent study. This was assumed to be acceptable as the purpose of these particular simulations were to determine the size where the mesh independence had been reached for the co-annular duct. The scenario of the finest mesh size had been performed with and without the moving valve to determine the effect on the velocity profile and ingress velocities. Two $\mathrm{He}_{-} \mathrm{SF}_{6}$ simulations were discussed in this paragraph as Case 3 and Case 4 in Table 3. The difference between Case 3 and Case 4 was the moving valve conditions. 


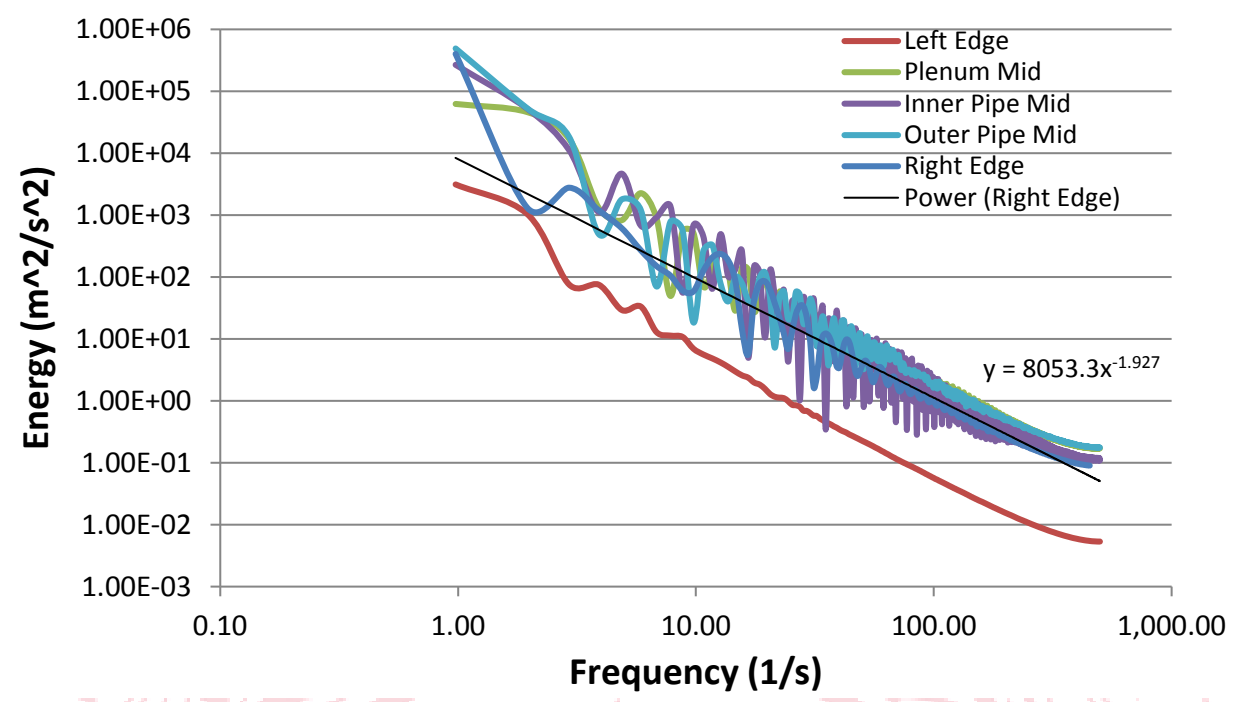

Figure 25. Frequency power spectra of the velocity evolutions at certain locations

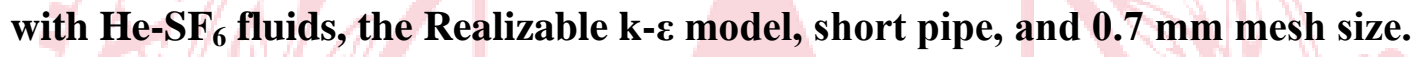

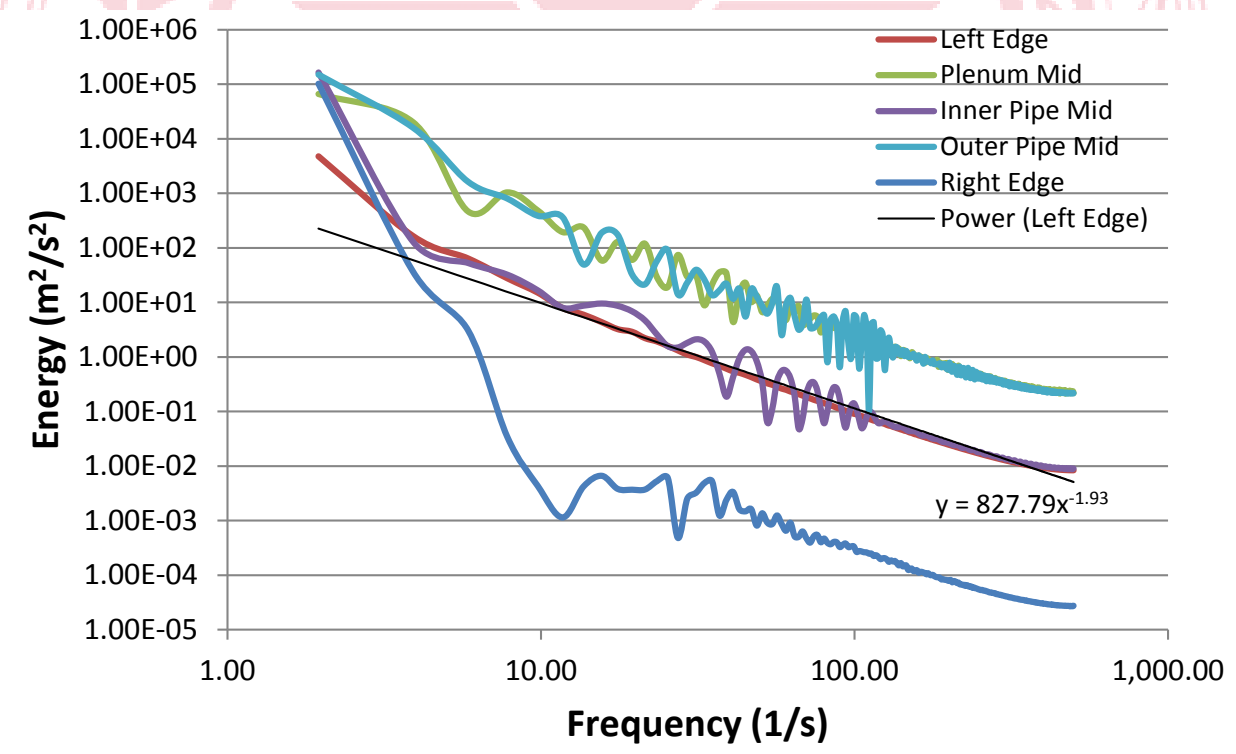

Figure 26. Frequency power spectra of the velocity evolutions at certain locations

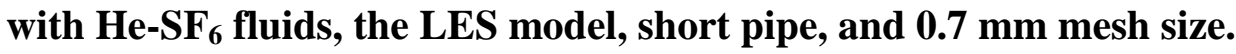


The fully open valve was assumed as the initial condition for Case 3 , and the valve opened with a constant velocity was implemented for Case 4 . The simulation and comparison results were seen in Table 3 and Table 4. From Case 3 of Table 3, the maximum wave front velocities of the inner and the outer pipe were $0.924 \mathrm{~m} / \mathrm{s}$ and $1.099 \mathrm{~m} / \mathrm{s}$, respectively. The scenario with the moving valve predicted the inner and the outer wave front velocity of $0.696 \mathrm{~m} / \mathrm{s}$ and $0.896 \mathrm{~m} / \mathrm{s}$, respectively. Based on the results, the ratio of the maximum velocity between the inner and the outer pipes is $84.1 \%$ for the fully open valve (Case 3). The ratio of the maximum wave front velocity of the inner pipe to the outer pipe was $77.7 \%$ (Eq. 5-4) for Case 4.

From Eq. 5-9, the absolute difference between the simulations (with and without the moving valve) was $0.225 \mathrm{~m} / \mathrm{s}$ and $0.199 \mathrm{~m} / \mathrm{s}$ for the inner pipe and the outer pipe, respectively. From Eq. 5-10, the relative differences of the wave front velocities for the inner and the outer pipes were calculated as $24.35 \%$ and $18.11 \%$, respectively. These calculations are listed in Table 4 . For the pipe length study, the difference in the waterbrine simulations was not as significant as that in the $\mathrm{He}_{-\mathrm{SF}_{6}}$ simulations.

$$
\begin{aligned}
& \left\{\begin{array}{l}
\Delta V_{\text {inner }}=V_{\text {inner }}(\text { no valve })-V_{\text {inner }}(\text { with valve }) \\
\Delta V_{\text {outer }}=V_{\text {outer }}(\text { no valve })-V_{\text {outer }}(\text { with valve })
\end{array}\right. \\
& \left\{\begin{array}{l}
\frac{\Delta V_{\text {inner }}}{V_{\text {inner }}(\text { no valve })}=100 \% \frac{V_{\text {inner }}(\text { no valve })-V_{\text {inner }}(\text { with valve })}{V_{\text {inner }}(\text { no valve })} \\
\frac{\Delta V_{\text {outer }}}{V_{\text {outer }}(\text { no valve })}=100 \% \frac{V_{\text {outer }}(\text { no valve })-V_{\text {outer }}(\text { with valve })}{V_{\text {outer }}(\text { no valve })}
\end{array}\right.
\end{aligned}
$$

\subsection{Wavelet Analysis}

The wavelet analyses were used to further analyze the simulation results. The simulation results of the gas-gas scenario with the long pipe geometry were used for the wavelet analyses because the temporal resolution was better. The Morlet mother wavelet function was implemented to study air-ingress phenomenon. Figure 27 gives an example of the instantaneous profile of the $\mathrm{SF}_{6}$ volume fraction. It was clear that more turbulence appeared to the wave fronts than the flow away from the wave head. The flow far away from the wave fronts was assumed to simply transport kinetic energy to the wave fronts in the large-frequency scales (or low frequencies). However, the low frequencies may contain other effects (e.g., the buoyancy frequency that will be discussed later in this section). Since it was inferred that the wave front head contained complex phenomena of flow transporting and energy cascading simultaneously, the wavelet analysis was used to help understand more information of the air-ingress phenomenon. Similarly, few positions were chosen for the 1D wavelet analysis. The Morlet mother function was applied for the 1D continuous transform. The results of continuous wavelet transform (CWT) with the corresponding velocity magnitude profiles are shown in Figure 28 and 
Figure 29. The decompositions in Figure 28 show slight difference of the dominant frequencies for different positions. The dominant frequency was approximately $1.7 \mathrm{~Hz}$ at the position near the valve and the center of the outer line-probe, and it was $3 \mathrm{~Hz}$ for the position at the left edge of the outer line-probe with a significantly secondary frequency as approximately $8 \mathrm{~Hz}$. Figure 29 shows that the dominant frequency was approximately $2 \mathrm{~Hz}$ for the position close to the valve in the inner line-probe and approximately $2.4 \mathrm{~Hz}$ for the position at the entrance of the light fluid tank and the left edge of the inner lineprobe. Three positions had secondary frequencies of $4 \mathrm{~Hz}$ and $8 \mathrm{~Hz}$.

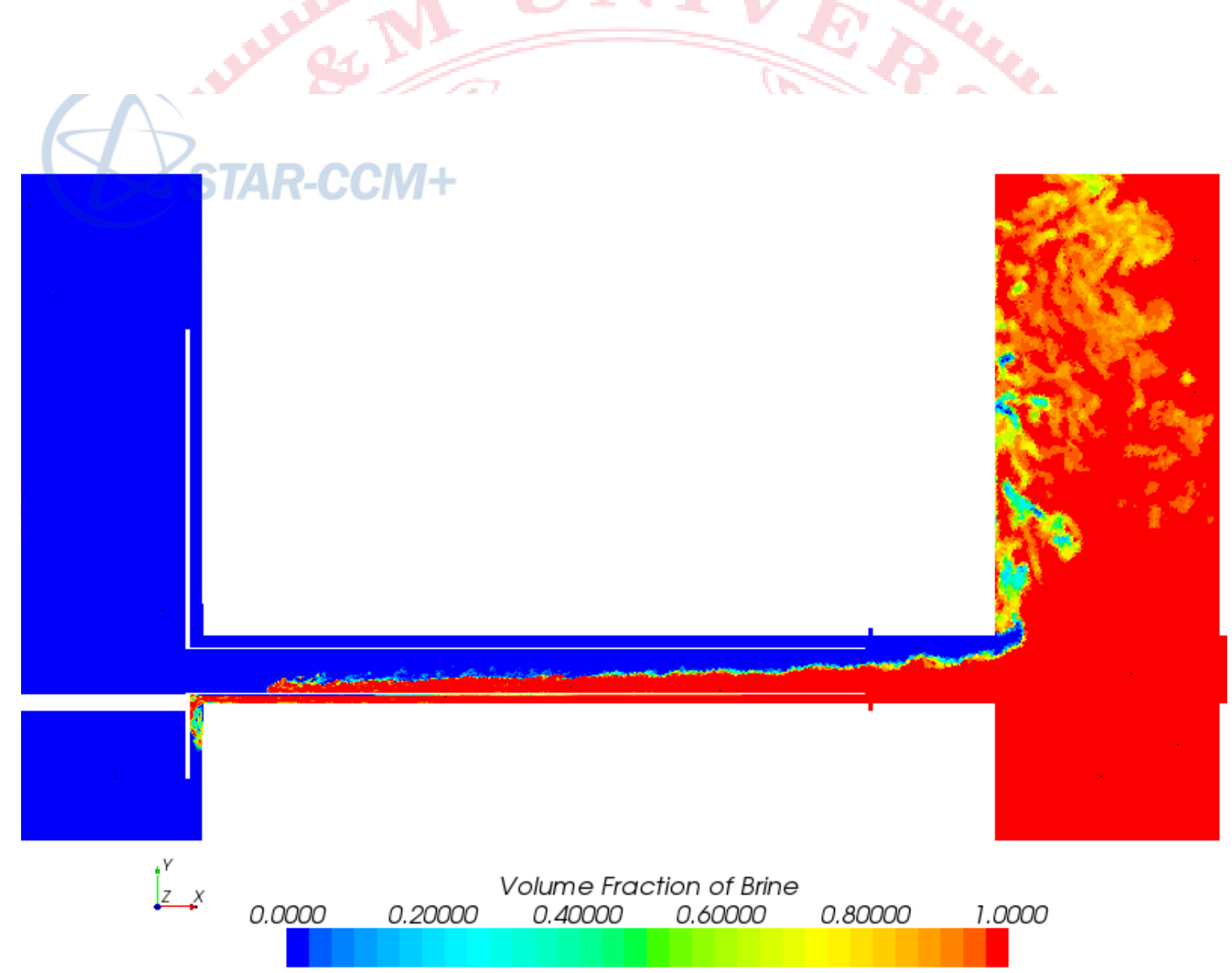

Figure 27. An example of volume fraction of Brine at the $6479^{\text {th }}$ time step. 

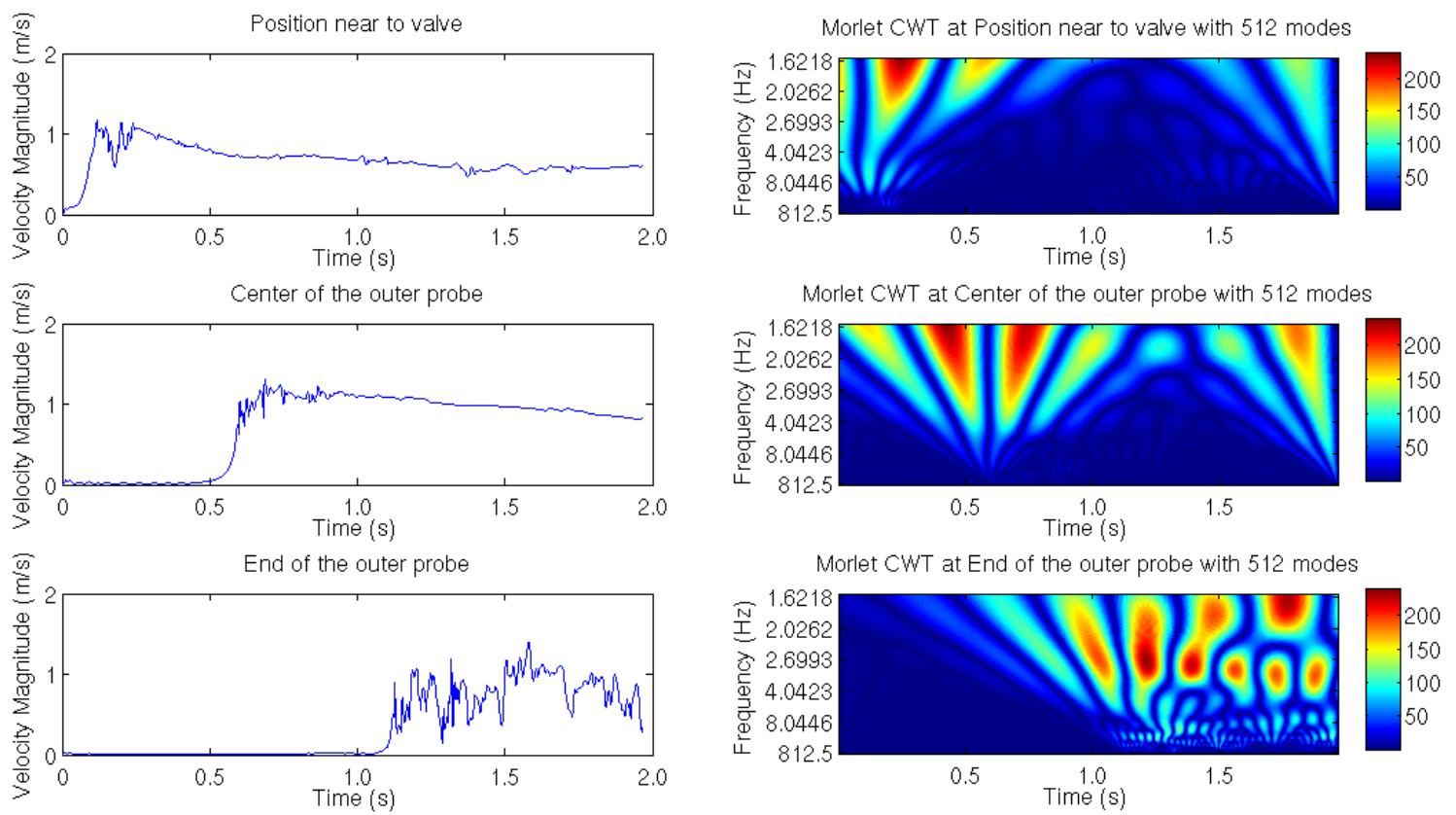

Figure 28. Instantaneous velocity magnitude signals from the outer line-probe of the long-pipe simulation results for the certain positions and their 1D continuous transform with using Morlet mother wavelet with 512 modes.
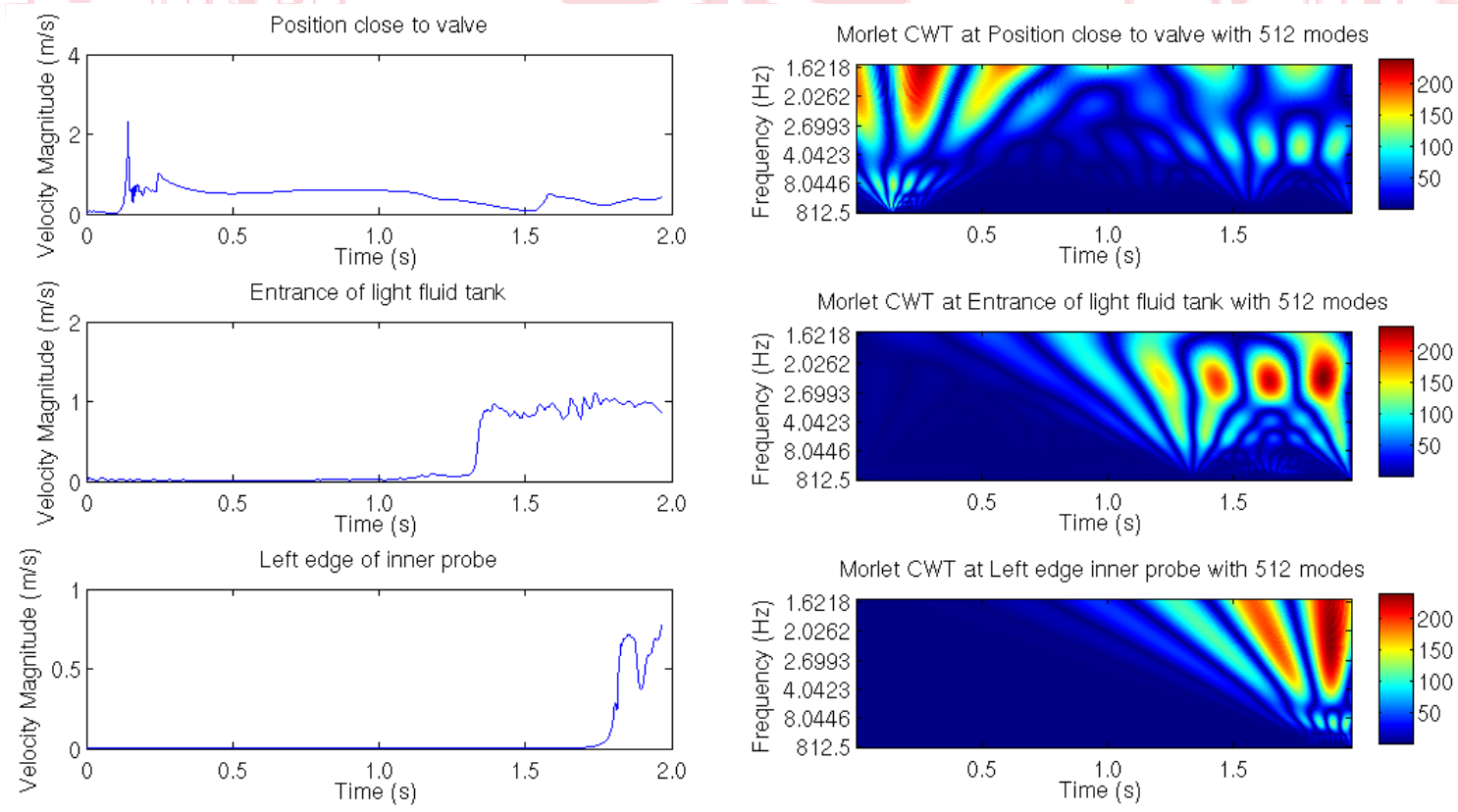

Figure 29. Instantaneous velocity magnitude signals from the inner line-probe of the long-pipe simulation results for the certain positions and their 1D continuous transform with using Morlet mother wavelet with 512 modes. 
In order to study the effects from the number of total modes used in the wavelet analysis, the numbers of the modes from 32 to 1024 were implemented for the 1D Morlet CWT shown in Figure 30. It was obvious that the more modes were used to decompose the signal, the smaller frequencies were able to be analyzed. In the results of using 32 modes of Morlet mother function, the larger frequencies were unable to capture any signals. The decomposed signals for these modes were indistinct to provide dominant frequency of the flow. The comparisons showed that the approximately $6 \mathrm{~Hz}$ as the dominant frequency from 128 modes was the secondary frequency presented in the results from 256 modes. Similarly, the results of using 512 modes contained the secondary frequencies of $6 \mathrm{~Hz}$ and showed approximately $2 \mathrm{~Hz}$ as its dominant frequency. When 1024 modes were selected for the $1 \mathrm{D}$ continuous transform, the previously secondary frequency of $6 \mathrm{~Hz}$ was hardly observed. Instead, the $2 \mathrm{~Hz}$ frequency became the secondary dominant frequency in the 1D Morlet CWT with 1024 modes where the dominant frequency was approximately $0.9 \mathrm{~Hz}$. As the total number of modes increases, the dominant frequency decreases (Figure 30).

The Brunt-Vaisala frequency, or buoyancy frequency, is given in Eq. 5-11.

$$
N \equiv \sqrt{-\frac{g}{\rho} \frac{d \rho}{d z}}
$$

where $\rho$ is potential density. If the density gradient is negative, the instability at the interface between two fluids will become oscillating solutions. However, if the density gradient is positive, the stratification will become unstable. From Eq. 5-11, the frequency was calculated as approximately $2.34 \mathrm{~Hz}$ at the end of the outer line-probe, or the left edge of the light fluid tank. The frequency was very close to the value $(2.4 \mathrm{~Hz})$ observed from the 1D Morlet wavelet analysis. The decomposition for this position in Figure 30 with 512 modes or 1024 modes confirmed this presence of a frequency in this range between times $1.2 \mathrm{~s}$ and $1.85 \mathrm{~s}$. 

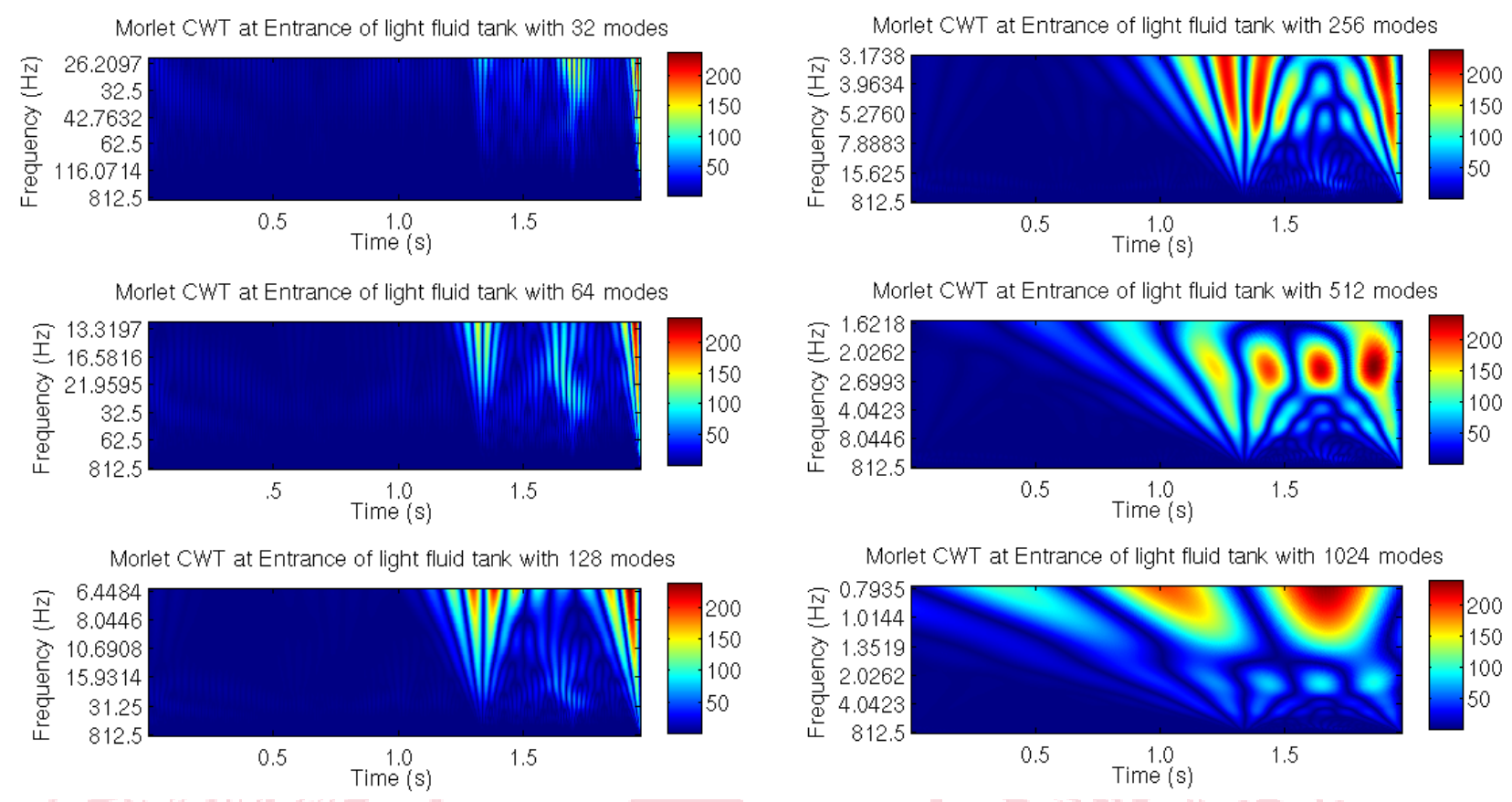

Figure 30. 1D Morlet CWT at the entrance of the light fluid tank with different number of modes from 32 to 1024.

\subsection{POD Method Analysis}

A plane cross-section shown in Figure 7 was utilized to extract results for the transient study with approximately 2 seconds. The LES results of approximately 2 seconds were used as the original modes of the database with a spatial resolution of 0.7 $\mathrm{mm}$ and a temporal resolution of one millisecond ( $1 \mathrm{~ms}) .80$ modes were selected to reconstruct the signal for comparisons with the original LES results. Generally, the first mode of POD indicates the mean flow profile and contains the most of the kinetic energy. The air ingress phenomenon is a strongly transient scenario, so the first mode may not have enough strong influences on the flow behaviors. Figure 31 shows the accumulation and the percentage of the kinetic energy of the flow for each mode. The first mode in this study contained the $65.2 \%$ of kinetic energy of the flow. The $99.1 \%$ of the kinetic energy of the flow was covered by the first 80 modes. As described in Section 5.1.2, POD can help filter information of small scales from the original signals and retain the main information of large scales. Unfortunately, the reconstructions were limited to obtain clear results for POD method because the air-ingress phenomenon was a strongly transient scenario. Figure 32 illustrates the example that the POD method provides limit efforts even though the total number of the modes used for the reconstruction is reduced. 
The signals in Figure 32 were extracted within the height of the wave front head (approximately $3 \mathrm{~mm}$ ) since the wave front was considered to have the most information. However, it was obvious that the reconstructions presented rarely provide useful comparisons with the original LES results. It can be inferred that the wave front head contain many features with large scales which make complex flow patterns at the wave front head distinguish from the original LES results. The mixing mechanism occurring near the wave front head resulted from the heavy fluid intrusions. The KelvinHelmholtz instability at the interface was caused by density difference or density gradient. Subsequently, the next study was focus on the interface of two fluids.

Figure 33 shows an example of the reconstruction with 80 modes and the original LES results. The 80 modes were selected because of $99.1 \%$ of the original signal. Only a slightly improvement was offered in the reconstruction with 80 modes that showed minor differences compared to the LES results. POD had a slightly better effort in the study of the fluid interface than in that of the wave front head.

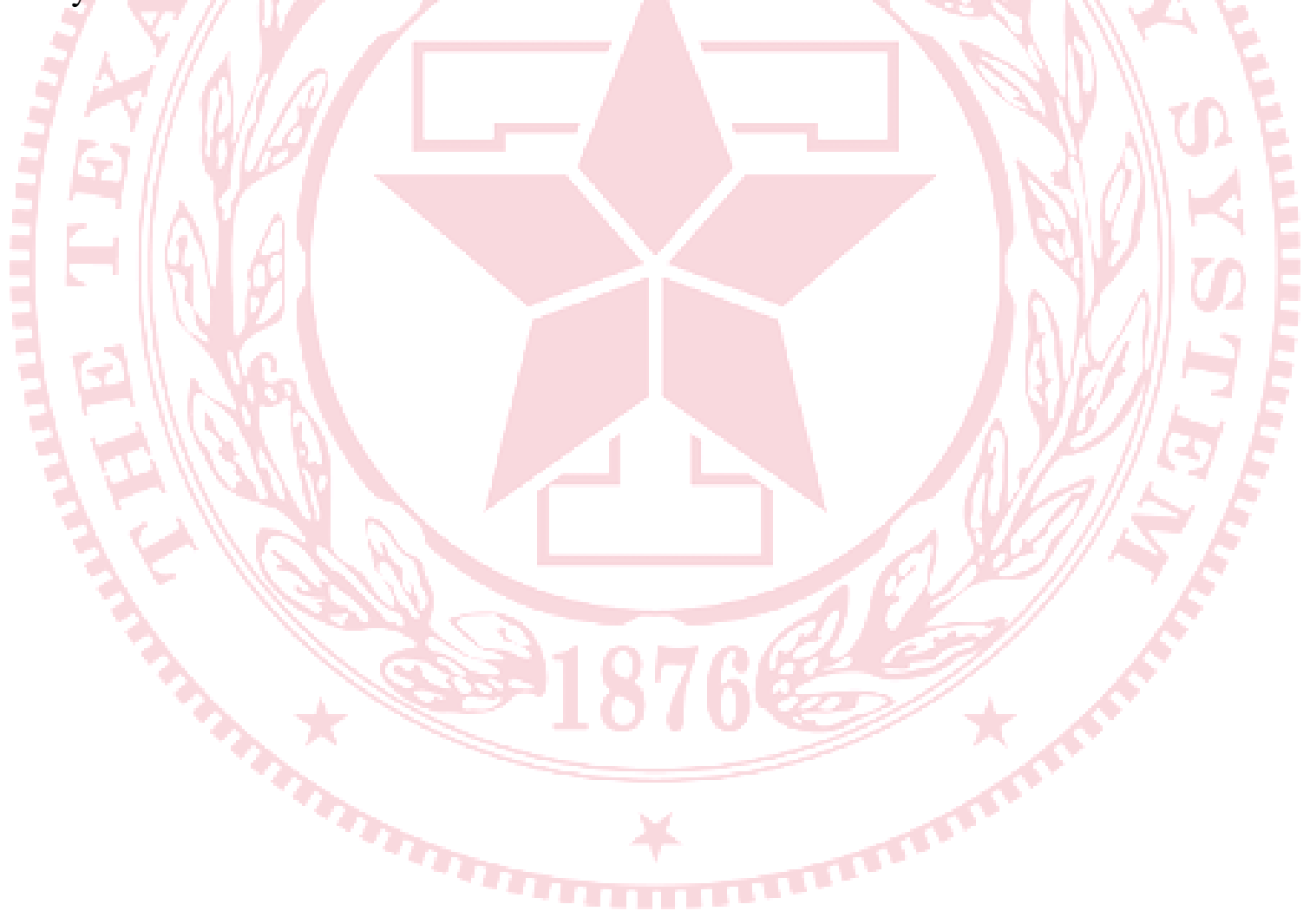




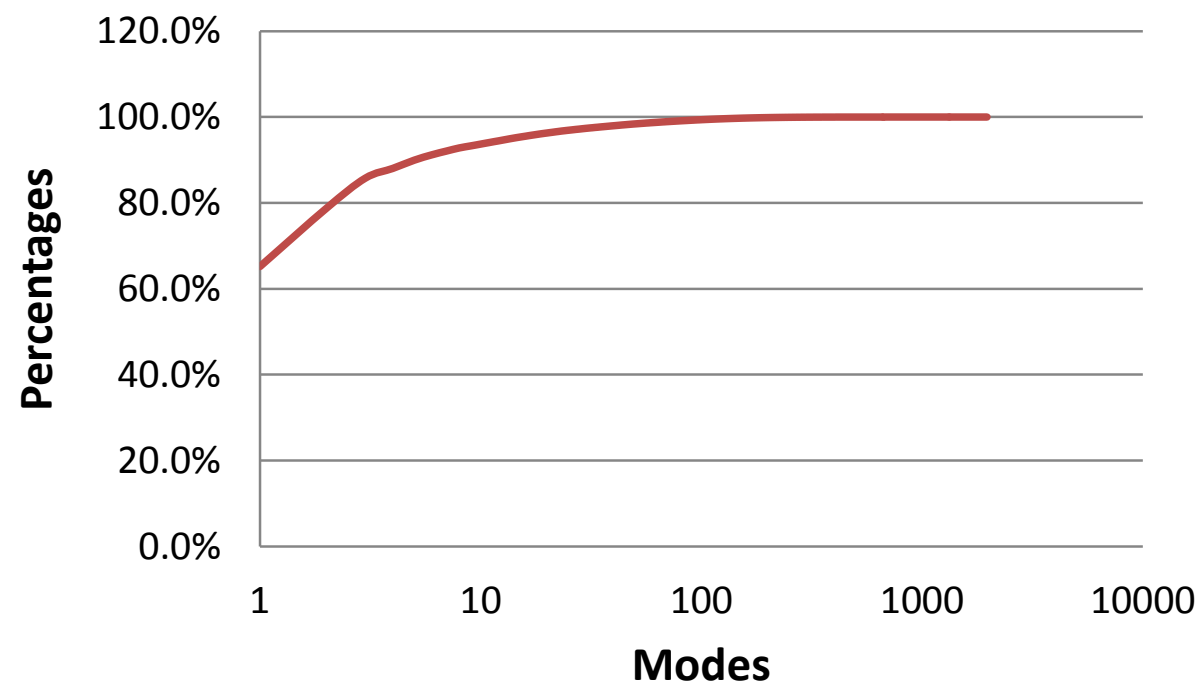

(a)

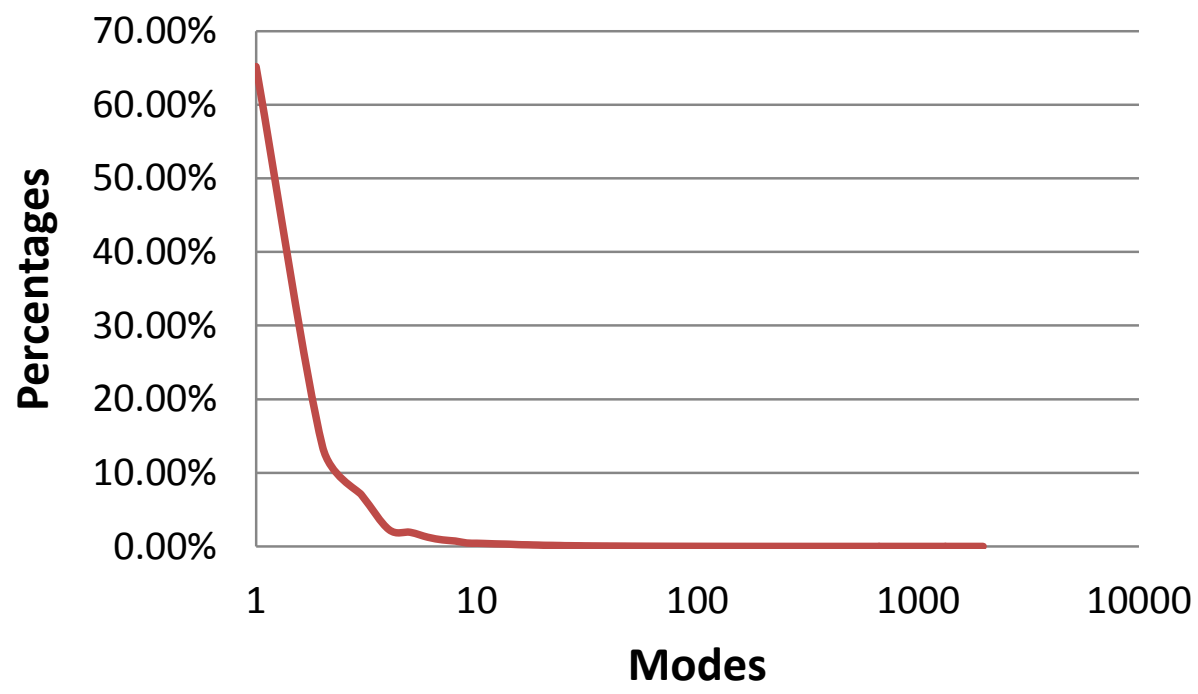

(b)

Figure 31. The percentage of total kinetic energy in each mode. (a) the accumulation percentage for each mode; (b) the percentage for each mode. The first mode contains the $65.2 \%$ of the kinetic energy of the flow. The first 80 modes contain $99.1 \%$. 
20 modes
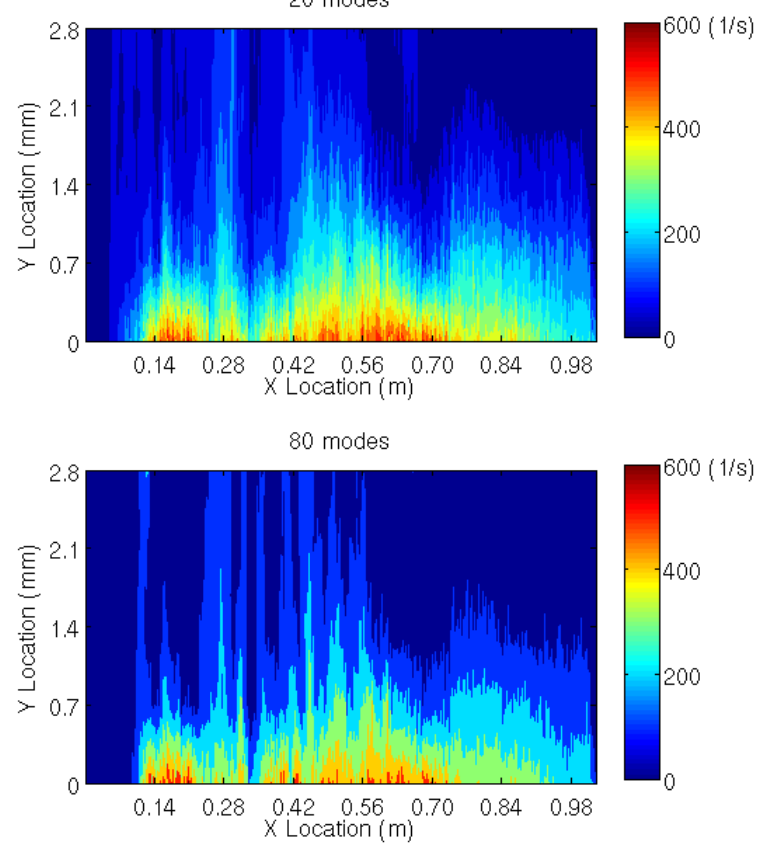

50 modes
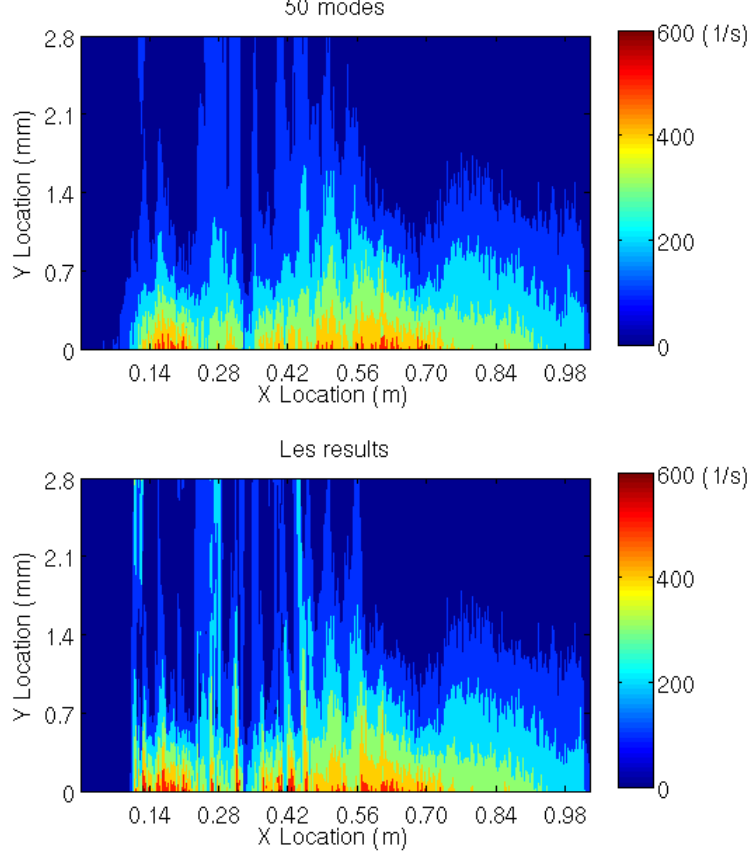

Figure 32. Vorticity magnitude contour of the $1200^{\text {th }}$ snapshot for the LES results and the reconstructions of the wave front head.

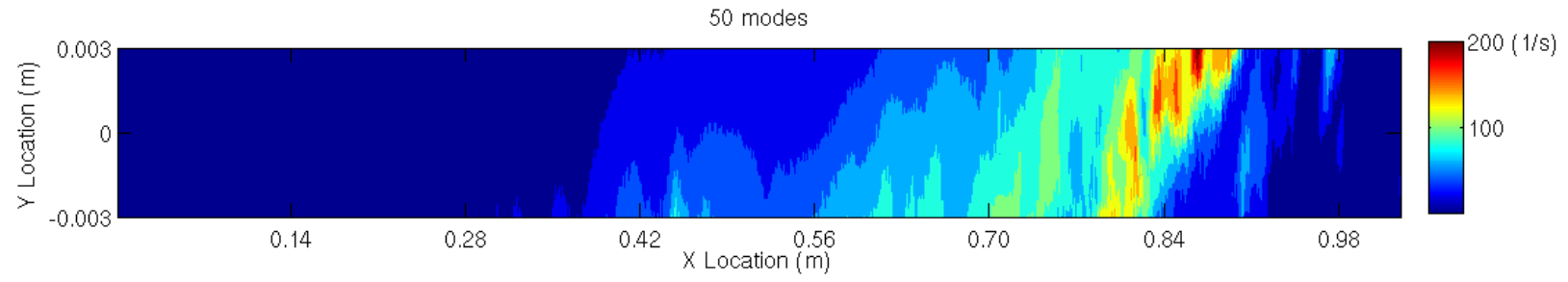

80 modes

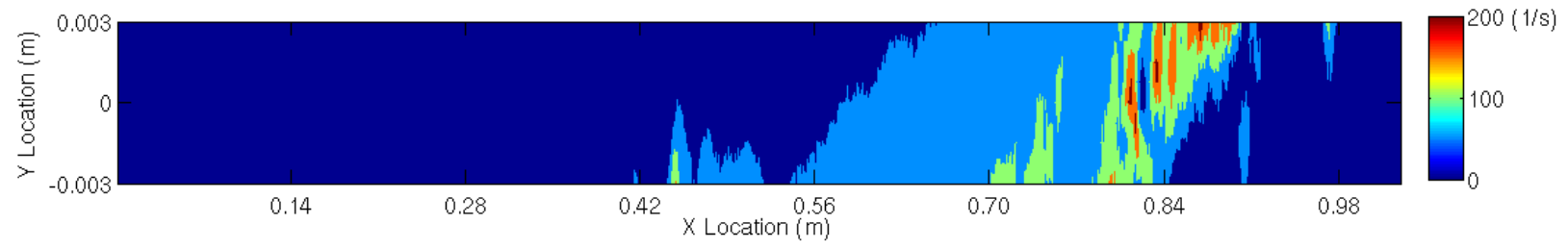

Les results

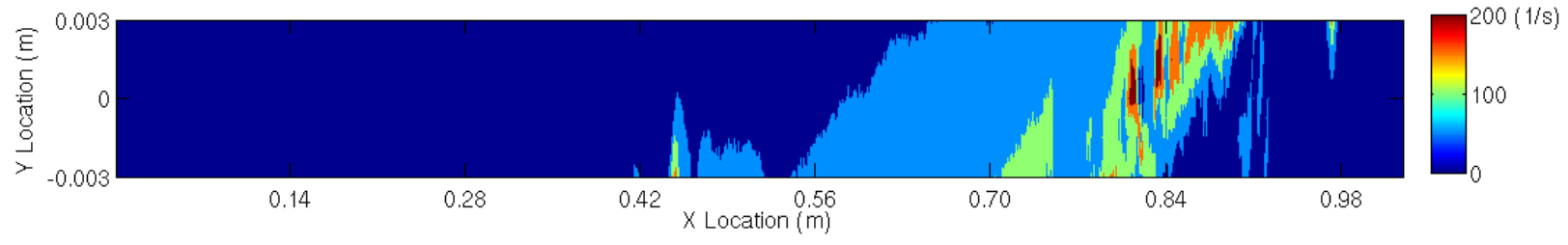

Figure 33. Vorticity magnitude contour of the $600^{\text {th }}$ snapshot for the reconstruction with 80 modes and the original LES results for the interface. 


\section{CHAPTER VI}

\section{CONCLUSION}

The Richardson extrapolation presented that the $0.7 \mathrm{~mm}$ mesh size had the relative errors within $1 \%$ for the grid independent study. For the simulations with the 0.7 $\mathrm{mm}$ mesh size, the RANS model showed good agreements with the LES approaches. The relative difference between the two turbulent models was approximately $3.32 \%$ for the inner pipe and $0.5 \%$ for the outer pipe. The moving valve scenarios with a constant speed for total $0.534 \mathrm{~s}$ decreased the maximum wave front velocities of $24 \%$ and $18 \%$ for the inner and the outer pipes, respectively. The assumption of the moving valve with a constant speed would be approximately appropriate if the first $0.534 \mathrm{~s}$ were not highly interesting. The simulation results showed very good agreements with the experimental data, and the relative errors were within $2.4 \%$. The pipe length which was used to mimic the break distance to the reactor enhanced the maximum wave front velocity approximately within $18 \%$ for the scenarios between the pipes of $0.38 \mathrm{~m}$ and $1 \mathrm{~m}$.

The POD method can find important components to reconstruct the signal. For the first few modes, it was able to obtain most of the information and the trend of the original signal. Not every mode is required since the last modes are insignificant. Once the modes increase enough, the reconstruction will be similar to the original results. In this study, the first eigenvalue contains the $85.4 \%$ of the kinetic energy of the flow. The remaining eigenvalues are regarded as turbulent kinetic energies. 1D Morlet wavelet analysis gave the dominant frequency as approximately $2.4 \mathrm{~Hz}$. This value was close to the calculation of using the buoyancy frequency which gave the value of approximately $2.34 \mathrm{~Hz}$. The CWT results with 512 modes or 1024 modes confirmed this frequency between times $1.2 \mathrm{~s}$ and $1.85 \mathrm{~s}$ in Figure 30.

Generally, the wavelet analysis has much better performance than the POD method in the air-ingress phenomenon which is a transient problem. The previous literatures done by the scientists and researchers were studied in the steady stratified flows. The POD method showed less information in a strongly transient problem. Based on this study, when the fluid pair in a real condition is used, the time for the intrusion of heavy fluids is predictable. 


\section{REFERENCES}

1. U.S. DOE Nuclear Energy Research Advisory Committee and the Generation IV International Forum, 2002, "A Technical Roadmap for Generation IV Nuclear Energy Systems," Generation IV International Forum.

2. "Generation IV Nuclear Energy Systems: Ten Year Program Plan", March 2005, Office of Advanced Nuclear Research, DOE Office of Nuclear Energy, Science, and Technology, U.S. DOE (2005).

3. "Next Generation Nuclear Plant Methods Technical Program Plan," INL/EXT-0611804, January, INL U.S. (2007).

4. N. E. Todreas, M. S. Kazimi, "Nuclear Systems I," Taylor \& Francis Group, NY, USA, (1990).

5. T. B. Benjamin, "Gravity currents and related phenomena," Journal of Fluid Mechanics, Vol. 31 part 2, pp. 209-248, (1968).

6. J. E. Simpson, "Effects of the lower boundary on the head of a gravity current," Journal of Fluid Mechanics, Vol. 61, pp. 731-751, (1972).

7. R. E. Britter and J. E. Simpson, "Experiments on the dynamics of a gravity current head," Journal of Fluid Mechanics, Vol. 88, pp. 223-240, (1978).

8. J. E. Simpson and R. E. Britter, "The dynamics of the head of a gravity current advancing over a horizontal surface," Journal of Fluid Mechanics, Vol. 94, part 3, pp. 477-495, (1979).

9. R. E. Britter and P. F. Linden, "The motion of the front of a gravity current travelling down an incline," Journal of Fluid Mechanics, Vol. 99, part. 3, pp. 531$543,(1980)$.

10. H. E. Huppert J. E. and Simpson, "The slumping of gravity currents," Journal of Fluid Mechanics, Vol. 99, pp. 785-799, (1980).

11. C. H. Gibson, "Fossil temperature, salinity, and vorticity in the ocean," Elsevier Oceanography Series, Vol. 28, pp. 221-257, (1980).

12. C. H. Gibson, "Buoyancy effects in turbulent mixing: sampling turbulence in the stratified ocean," AIAA, Vol. 19, No. 11, pp. 1394-1400, (1981).

13. D. L. Wilkinson, "Motion of air cavities in long horizontal ducts," Journal of Fluid Mechanics, Vol. 118, pp. 109-122,(1982).

14. G. C. Gardner and I. G. Crow, "The motion of large bubbles in horizontal channels," Journal of Fluid Mechanics, Vol. 43, pp. 247-255, (1970).

15. J. W. Rottman and J. E. Simpson, "Gravity currents produced by instantaneous releases of a heavy fluid in a rectangular channel," Journal of Fluid Mechanics, Vol. 135, pp. 95-110, (1983). 
16. C. A. G. Webster, "An experimental study of turbulence in a density-stratified shear flow," Journal of Fluid Mechanics, Vol. 19, pp. 221-245, (1964).

17. J. T. Lin and G. J. Binder, "Simulation of mountain lee waves in a wind tunnel," College of Engng, Colorado State University Int. Rep. CER67-88 JTL-6JB24, (1967).

18. R. S. Scotti, "An experimental study of a stratified free shear layer," College of Engineering, University of California, Berkerly Rep. no. AS-69-1 (1969).

19. R. S. Scotti and G.M. Corcos, "An experiment on the stability of small disturbances in a stratified free shear layer," Journal of Fluid Mechanics, Vol. 52, part 3, pp. 499-528, (1972).

20. Y. H. Pao, "Measurements of internal waves and turbulence in two-dimensional stratified shear flows," Boundary Layer Met. 5, pp. 177-193, (1973).

21. R. E. Lange, "Decay of turbulence in stratified salt water," Ph.D. thesis, University of California, San Diego, (1974).

22. T. D. Dickey and G. L. Mellor, "Decaying turbulence in neutral and stratified fluids," Journal of Fluid Mechanics, Vol. 99, part 1, pp. 13-31, (1980).

23. D. C. Stillinger, M. J. Head, K. N. Helland and C. W. Van Atta, "A close-loop gravity-driven water channel for density-stratified shear flows," Journal of Fluid Mechanics, Vol. 131, pp. 73-89, (1983).

24. H. P. Grobelbauer, T. K. Fannelop and R. E. Britter, "The propagation of intrusion fronts of high density ratios," Journal of Fluid Mechanics, Vol. 250, pp. 669-687, (1993).

25. D. Barnea and Y. Taitel, "Kelvin-Helmholtz stability criteria for stratified flow: viscous versus non-viscous (inviscid) approaches," Int. J. Multiphase flow, Vol. 19, No. 4, pp. 639-649, (1993).

26. C. Hartel, E. Meiburg and F. Necker, "Analysis and direct numerical simulation of the flow at a gravity-current head. Part 1 Flow topology and front speed for slip and no-slip boundaries," Journal of Fluid Mechanics, Vol. 418, pp. 189-212, (2000).

27. J. O. Shin, S. B. Dalziel, and P. F. Linden, "Gravity currents produced by lock exchange," Journal of Fluid Mechanics, Vol. 521, pp. 1-34. (2004).

28. H.Y. Gu and L.J. Guo,"Stability of stratified gas-liquid flow in horizontal and near horizontal pipes," Chin. J. Eng., Vol. 15(5), pp. 619-625, (2007).

29. D. D. Stretch, J. W. Rottman, S. K. Venayagamoorthy, K. K. Nomura, and C. R. Rehmann, "Mixing efficiency in decaying stably stratified turbulence," Dynamics of Atmospheres and Oceans, DYNAT-803 No. 12, (2009).

30. R. J. Lowe, J. W. Rottman and P. F. Linden, "The non-Boussinesq lock-exchange problem. Part 1. Theory and experiments," Journal of Fluid Mechanics, Vol. 537, pp. 101-124, (2005). 
31. V. K. Birman, J. E. Martin and E. Meiburg, "The non-Boussinesq lock-exchange problem. Part 2. High-resolution simulations," Journal of Fluid Mechanics, Vol. 537, pp. 125-144, (2005).

32. C. H. Oh, R. L. Moore, B. J. Merrill, and D. A. Petti, "Air ingress analyses on a high temperature gas-cooled reactor," INEEL/CON-01-00581, Nov. (2001).

33. C. H. Oh, E. S. Kim, H. S. Kang, H. C. NO, and N. Z. Cho, "Experimental Validation of Stratified Flow Phenomena, Graphite Oxidation, and Mitigation Strategies of Air Ingress Accidents," INL/EXT-08-14840, Idaho National Laboratory, U.S., Dec. (2009).

34. IAEA, "Heat transport and afterheat removal for gas cooled reactors under accident conditions," IAEA-TECDOC-1163, (2000).

35. C. H. Oh, C. Davis, L. Siefken, R. Moore, H. NO, J. Kim, G. C. Park, J. Lee, and W. Martin, "Development of Safety Analysis Codes and Experimental Validation for a Very High Temperature Gas-Cooled Reactor," Final Report, INL/EXT-06-01362, Idaho National Laboratory. U.S., (2006).

36. C. H. Oh and E. S. Kim, "Validations of CFD code for density-gradient driven air ingress stratified flow," ICONE 18, May (2008).

37. C. H. Oh and E. S. Kim, "Air ingress analysis: computational fluid dynamic models," IHTC14-23083, Aug. (2010).

38. GENERAL ATOMICS 1996, "Gas turbine-modular helium reactor (GT-MHR) Conceptual design description report," GA-A910720, Project (1996).

39. J. Hartley, "Double Ended Guillotine Break in a Prismatic Block VHTR Lower Plenum Air Ingress Scenario" MS thesis, Texas A\&M University, TX, USA (2011).

40. STAR-CCM+ USER GUIDE Version 5.02, CD-adapco, (2009).

41. ANSYS-CFX USER GUIDE Version 13.0, ANSYS Inc., (2010).

42. ANSYS-FLUENT GUIDE Version 13.0, ANSYS Inc., (2010).

43. MATLAB USER GUIDE Version 7.11.0, (R2010b).

44. D.T. Lee and A. Yamamoto, "Wavelet analysis: theory and applications," HewlettPackard Journal, pp. 44-54, Dec. (1994).

45. E. Merzari, H. Ninokata, "Large Eddy Simulation and Proper Orthogonal Decomposition of the flow in Annular Channnels." Annual Report of the Earth Simulator Center, Chapter 3 (April 2007- March 2008).

46. R. J. Adrian, K. T. Christensen, Z.-C. Liu, "Analysis and interpretation of instantaneous turbulent velocity fields," Experiment in Fluids, Vol. 29, pp. 275-290, (2000).

47. D. Hilberg, W. Lazik, and H.E. Fiedler, "The application of classical POD and snapshot POD in a turbulent shear layer with periodic structures," Applied Scientific Research, Vol. 53, pp. 283-290, (1994). 
48. S. B. Pope, "Turbulent Flows," Chapter 6, pp. 184-235, ISBN: 0-521-59886-9 Cambridge University, UK (2000).

49. M. Germano, "Turbulence: the filtering approach." Journal of Fluid Mechanics, Vol. 238, pp. 325-336, (1992).

50. H.C. Wei, J. Hartley, Y. A. Hassan, "CFD simulation of air-ingress problem following a double-ended guillotine break in GT-MHR," ANS Meeting Winter 2011.

51. C. J. Roy, "Grid Convergence Error Analysis for Mixed-Order Numerical Schemes," AIAA, Vol. 41, No. 4, pp. 595-604, (2003).

52. P. J. Roache, "Verification and validation in computational science and engineering," Hermosa, Albuquerque, NM, USA, (1998).

53. "Guide for the Verification and Validation of Computational Fluid Dynamics Simulations," AIAA-G-077-1998, AIAA, Reston, VA (1998).

54. L. F. Richardson, "The deferred approach to the limit," Transactions of the Royal Society of London, Series A, Vol. 226, pp. 229-361 (1927).

55. P. J. Roache, "Perspective: A method for uniform reporting of grid refinement studies," Journal of Fluids Engineering, Vol. 116, No. 3, pp. 405-413, (1994). 